\title{
Supporting Information for \\ Sequence-Defined Dendrons Dictate Supramolecular Cogwheel Assembly of Dendronized Perylene Bisimides
}

Benjamin E. Partridge, ${ }^{\dagger}$ Li Wang, ${ }^{\dagger \dagger}$ Dipankar Sahoo, ${ }^{\dagger}$ James T. Olsen, ${ }^{\dagger}$ Pawaret Leowanawat, ${ }^{\dagger}$ Cecilé Roche, ${ }^{\dagger}$ Henrique Ferreira, ${ }^{\dagger}$ Kevin J. Reilly, ${ }^{\dagger}$ Xiangbing Zeng, ${ }^{\S}$ Goran Ungar,${ }^{\S} \perp$ Paul A. Heiney, ${ }^{\#}$ Robert Graf, ${ }^{\diamond}$ Hans W. Spiess, ${ }^{\diamond}$ and Virgil Percec ${ }^{\dagger, *}$

${ }^{\dagger}$ Roy \& Diana Vagelos Laboratories, Department of Chemistry, University of Pennsylvania, Philadelphia, Pennsylvania 191046323, United States

* College of Materials Science and Engineering, Beijing University of Chemical Technology, Beijing 100029, China.

${ }^{\S}$ Department of Materials Science and Engineering, University of Sheffield, Sheffield, S1 3JD, United Kingdom

$\perp$ State Key Laboratory for Mechanical Behavior of Materials, Xi' an Jiaotong University, Xi' an 710049, China

\# Department of Physics and Astronomy, University of Pennsylvania, Philadelphia, Pennsylvania 19104-6396, United States

${ }^{\diamond}$ Max-Planck Institute for Polymer Research, 55128 Mainz, Germany

*E-mail: percec@sas.upenn.edu

Table of Contents

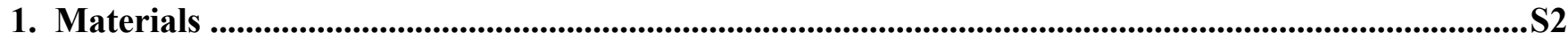

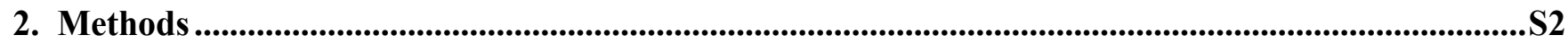

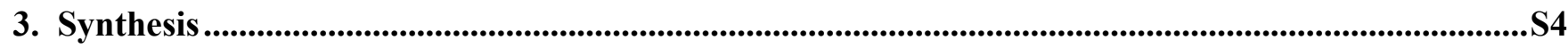

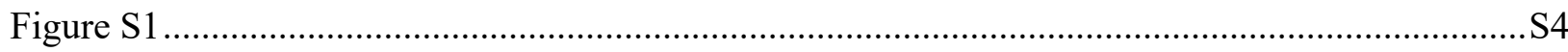

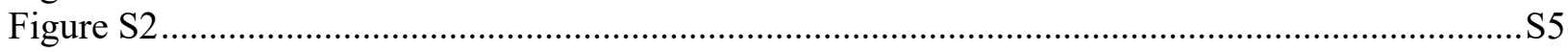

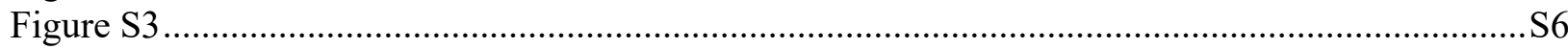

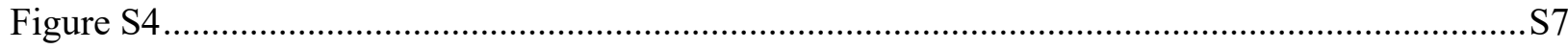

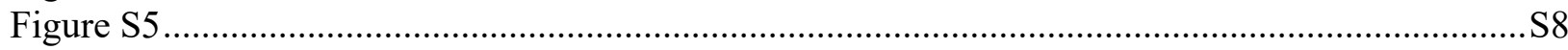

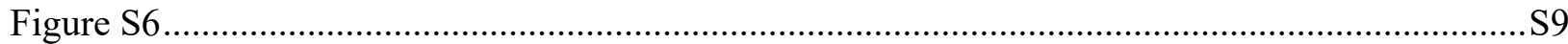

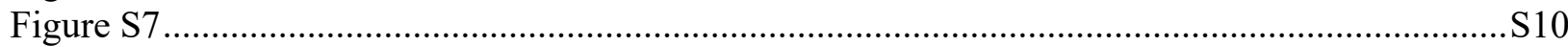

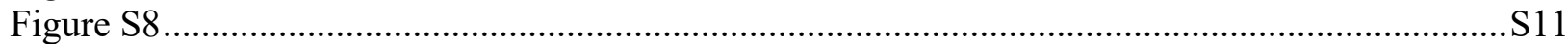

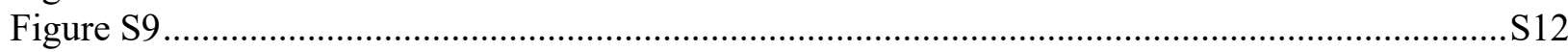

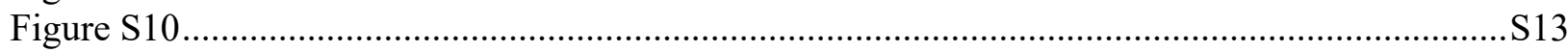

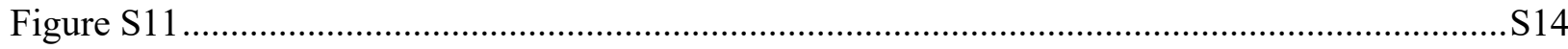

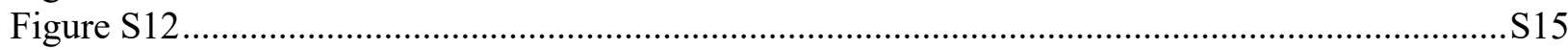

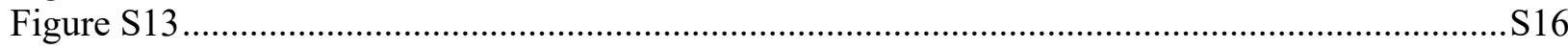

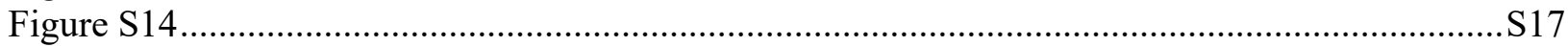

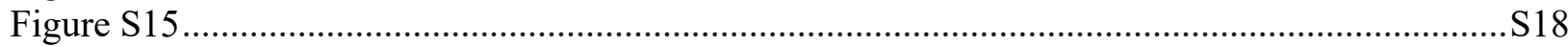

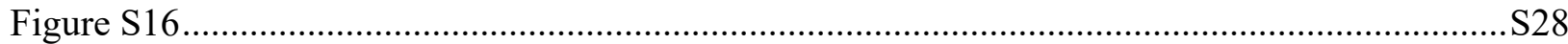

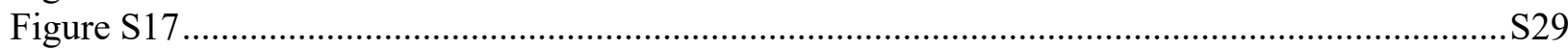

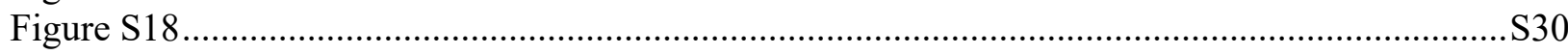

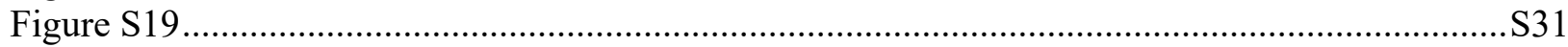

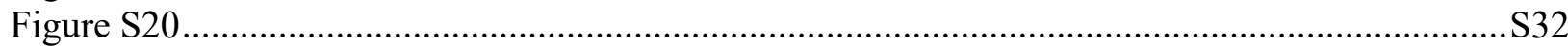

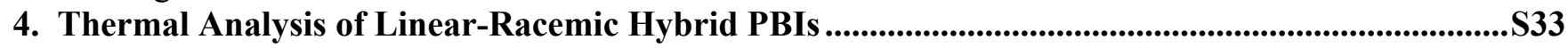

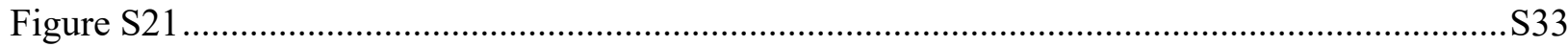

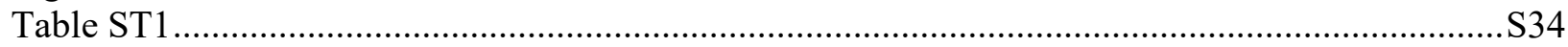

5. Structural and Retrostructural Analysis of Linear-Racemic Hybrid PBIs .........................................S35

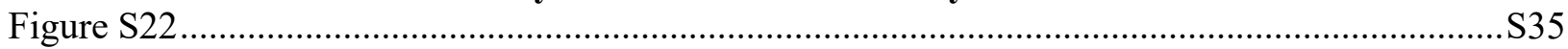

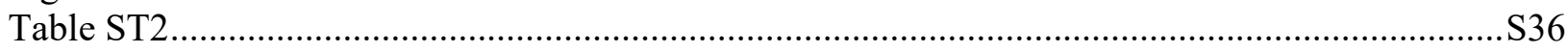

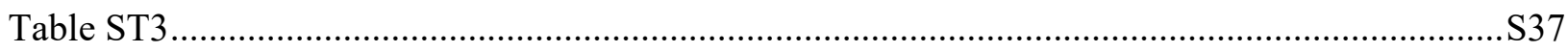

6. Solid State ${ }^{13}$ C CP-MAS NMR of Linear-Racemic Hybrid PBIs ..........................................................S38

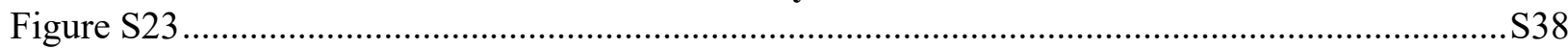

7. Representative Models of Linear-Racemic Hybrid PBIs in the $\Phi_{h}{ }^{k 2}$.............................................S39

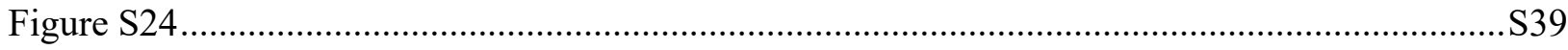

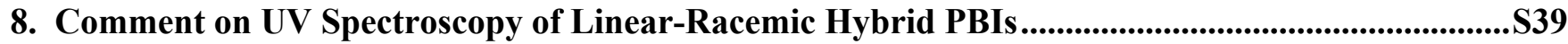

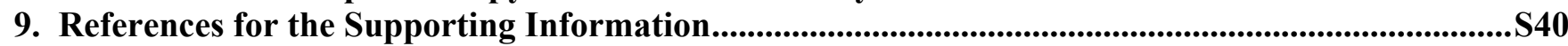




\section{Materials}

Gallic acid (Chem Impex, anhydrous, ACS grade), methanol (Fisher, ACS grade), concentrated sulfuric acid (Fisher, ACS grade), $\mathrm{NaHCO}_{3}$ (Fisher, ACS grade), ethyl acetate (Fisher, ACS grade), anhydrous $\mathrm{MgSO}_{4}$ (Oakwood Chemical, anhydrous), benzyl chloride (Alfa Aesar, 99\%), $\mathrm{K}_{2} \mathrm{CO}_{3}$ (Chem Impex, anhydrous, 99.75\%), KI (Alfa Aesar, 99\%), acetone (Fisher, ACS grade), $\mathrm{NaCl}$ (EMD Millipore Corporation, ACS grade), hexanes (Fisher, ACS grade), 1-bromooctane (Aldrich, 99\%), (rac)-1-bromo-3,7-dimethyloctane (Tokyo Chemical Industry Co. Ltd., 93+\%), palladium on activated carbon catalyst (Aldrich, $10 \mathrm{wt} \%$ loading), hydrogen (Airgas), $\mathrm{LiAlH}_{4}$ (Acros, 95\%), NaOH (VWR Analytical, ACS grade), thionyl chloride (Alfa Aesar, 99.5\%), $\mathrm{NaN}_{3}$ (VWR Life Science), perylenetetracarboxylic acid bisanhydride (PTCDA, Acros, 98\%), $\mathrm{Zn}(\mathrm{OAc})_{2} \cdot 2 \mathrm{H}_{2} \mathrm{O}$ (Acros, 98+\%), quinoline (Acros, 99\%), concentrated hydrochloric acid (Fisher, ACS grade), triethyl orthoformate (TCI, 98+\%), Amberlyst-15(H) (Alfa Aesar), toluene (Fisher, ACS grade), and silica gel (60 A, 32-63 $\mu \mathrm{m}$, Sorbent Technology) were used as received. Dichloromethane (DCM, Fisher, ACS grade) was refluxed over $\mathrm{CaH}_{2}$ and freshly distilled before use. THF (Fisher, ACS grade) was refluxed over sodium/benzophenone until the solution turned purple and distilled before use. Dimethylformamide (Fisher, ACS grade) was dried over $4 \AA$ molecular sieves prior to use.

\section{Methods}

NMR. ${ }^{1} \mathrm{H}$ NMR and ${ }^{13} \mathrm{C}$ NMR spectra were recorded on a Bruker UNI 500 or Bruker Neo 400 instrument at $300 \mathrm{~K}$ using the indicated deuterated solvent.

HPLC. The purity of the products was determined by a combination of thin-layer chromatography (TLC) on silica gel coated plates and high-performance liquid chromatography (HPLC) using THF as mobile phase at 1 $\mathrm{mL} / \mathrm{min}$, on a Shimadzu LC-10AT high-performance liquid chromatograph equipped with a Perkin Elmer LC100 oven $\left(40^{\circ} \mathrm{C}\right)$, containing two Perkin-Elmer PL gel columns of $5 \times 10^{2}$ and $1 \times 10^{4} \AA$, a Shimadzu SPD10A UV detector $(\lambda=254 \mathrm{~nm})$, a Shimadzu RID-10A RI-detector, and a PE Nelson Analytical 900 Series integrator data station.

Differential scanning calorimetry (DSC). Thermal transitions were determined on a TA Instruments Q100 differential scanning calorimeter (DSC) equipped with a refrigerated cooling system at heating and cooling rates of $10^{\circ} \mathrm{C} / \mathrm{min}$. Indium was used as calibration standard. The transition temperatures were calculated as the maxima and minima of their endothermic and exothermic peaks. An Olympus BX51 optical microscope (100× magnification) equipped with a Mettler FP82HT hot stage and a Mettler Toledo FP90 Central Processor was used to verify thermal transitions. Melting points were measured using a uni-melt capillary melting point apparatus (Arthur H. Thomas Company).

Density measurements. A small extruded fiber $(\sim 1.0 \mathrm{mg})$ was placed in a vial filled with water. The sample sank to the bottom of the vial due to its higher density compared with water. A saturated aqueous solution of sodium chloride was then added into the solution at $\sim 0.1 \mathrm{~g}$ per aliquot to gradually increase the solution density. Sodium chloride solution was added at an interval of at least $20 \mathrm{~min}$ to ensure equilibrium within the solution. 
When the sample was suspended in the middle of the solution, the density of the sample was identical to that of the solution, which was measured by a $10 \mathrm{~mL}$ volumetric flask.

Matrix-assisted laser desorption/ionization time of flight (MALDI-TOF) mass spectrometry. MALDI-TOF mass spectrometry was performed on PerSeptive Biosystems-Voyager-DE (Framingham, MA) mass spectrometer equipped with a nitrogen laser $(337 \mu \mathrm{m})$ and operated in linear mode. Internal calibration was performed using Angiotensin II, and Bombesin as standards. The analytical samples were obtained by mixing the THF solutions of samples $(5-10 \mathrm{mg} / \mathrm{mL})$ with the matrix solution (3,5-dihydroxybenzoic acid) $(10 \mathrm{mg} / \mathrm{mL})$ in a $1: 1$ to $1: 5 \mathrm{v} / \mathrm{v}$ ratio. The prepared solution $(0.5 \mu \mathrm{L})$ was loaded on a MALDI plate and allowed to dry at $25{ }^{\circ} \mathrm{C}$ before the plate was inserted into the vacuum chamber of the MALDI-TOF instrument. The laser intensity and voltage were adjusted depending on the molecular weight, and the nature of each analyte.

$X$-ray diffraction $(X R D)$. X-ray diffraction (XRD) measurements were performed using $\mathrm{Cu}-\mathrm{K}_{\alpha 1}$ radiation $(\lambda=$ $1.542 \AA$ ) from a Bruker-Nonius FR-591 rotating anode X-ray source equipped with a $0.2 \times 0.2 \mathrm{~mm}^{2}$ filament and operated at $3.4 \mathrm{~kW}$. Osmic Max-Flux optics, and triple pinhole collimation were used to obtain a highly collimated beam with a $0.3 \times 0.3 \mathrm{~mm}^{2}$ spot on a Bruker-AXS Hi-Star multiwire area detector. To minimize attenuation, and background scattering, an integral vacuum was maintained along the length of the flight tube, and within the sample chamber. Samples were held in glass capillaries (1.0 $\mathrm{mm}$ in diameter), mounted in a temperature-controlled oven (temperature precision: $\pm 0.1^{\circ} \mathrm{C}$, temperature range: from -10 to $210^{\circ} \mathrm{C}$ ). Aligned samples for fiber XRD experiments were prepared using a custom-made extrusion device. ${ }^{1}$ Fibers were extruded at $23{ }^{\circ} \mathrm{C}$ with an extrusion time of less than $0.5 \mathrm{~s}$. Powdered sample $(\sim 10 \mathrm{mg})$ was heated inside the extrusion device. After slow cooling, the fiber was extruded in the liquid crystal phase, and cooled to $23{ }^{\circ} \mathrm{C}$. Typically, the aligned samples have a thickness of $0.3-0.7 \mathrm{~mm}$, and a length of 3-7 mm. All XRD measurements were done with the aligned sample axis perpendicular to the beam direction. Sample-to-detector distance was $0.07 \mathrm{~m}$. Additional measurements to verify indexed parameters were performed with a sampleto-detector distance of $0.54 \mathrm{~m}$. Primary XRD analysis was performed using Datasqueeze (version 3.0.5). ${ }^{2}$ 


\section{Synthesis}

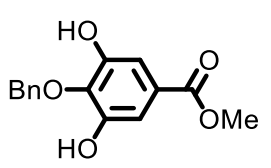

Methyl 4-(benzyloxy)-3,5-dihydroxybenzoate (2). Following a procedure reported by Pearson et al., ${ }^{3}$ a mixture of $1(10.00 \mathrm{~g}, 54.3 \mathrm{mmol}), \mathrm{K}_{2} \mathrm{CO}_{3}(9.00 \mathrm{~g}, 65.1 \mathrm{mmol})$ and $\mathrm{KI}(1.5 \mathrm{~g}, 9.0 \mathrm{mmol})$ was stirred in acetone $(300 \mathrm{~mL})$ under $\mathrm{N}_{2}$ protection. Benzyl chloride $(7.5 \mathrm{~mL}, 65.1 \mathrm{mmol})$ was added and the reaction mixture was heated under reflux for $15 \mathrm{~h}$. Then the mixture was cooled, concentrated to about $50 \mathrm{~mL}$, poured into water $(300 \mathrm{~mL})$ and extracted with EA $(100 \mathrm{~mL} \times 5)$. The combined EA phase was washed with brine $(100 \mathrm{~mL} \times 2)$, dried over anhydrous $\mathrm{MgSO}_{4}$, filtered and evaporated in vacuo. Column chromatography $(\mathrm{EA} /$ Hexanes $=1 / 4)$ gave 3 as a white solid. Product: 5.25 g. Yield: 35\%. Purity by HPLC: $99+\%$. TLC (EA/Hexanes $=1 / 2): \mathrm{R}_{\mathrm{f}}=0.33$. M. P.: 131-133 ${ }^{\circ} \mathrm{C}$.

${ }^{1} \mathrm{H}$ NMR (500 MHz, $\left.\mathrm{CDCl}_{3}, \delta\right): 7.41-7.38\left(\mathrm{~m}, 5 \mathrm{H},-\mathrm{OC}_{6} H_{5}\right), 7.23$ (s, 2H, ArH ortho to $\left.\mathrm{CO}_{2} \mathrm{CH}_{3}\right), 5.59$ (s, $2 \mathrm{H}, \mathrm{ArOH}), 5.14$ (s, 2H, $\left.-\mathrm{OCH} H_{2} \mathrm{Ar}\right), 3.89$ (s, 3H, $\left.-\mathrm{CO}_{2} \mathrm{CH}_{3}\right) .{ }^{13} \mathrm{C} \mathrm{NMR}\left(125 \mathrm{MHz}, \mathrm{CDCl}_{3}, \delta\right): 166.66$, $149.44,137.51,136.87,128.57,128.54,125.72,109.30,51.93,30.02$.
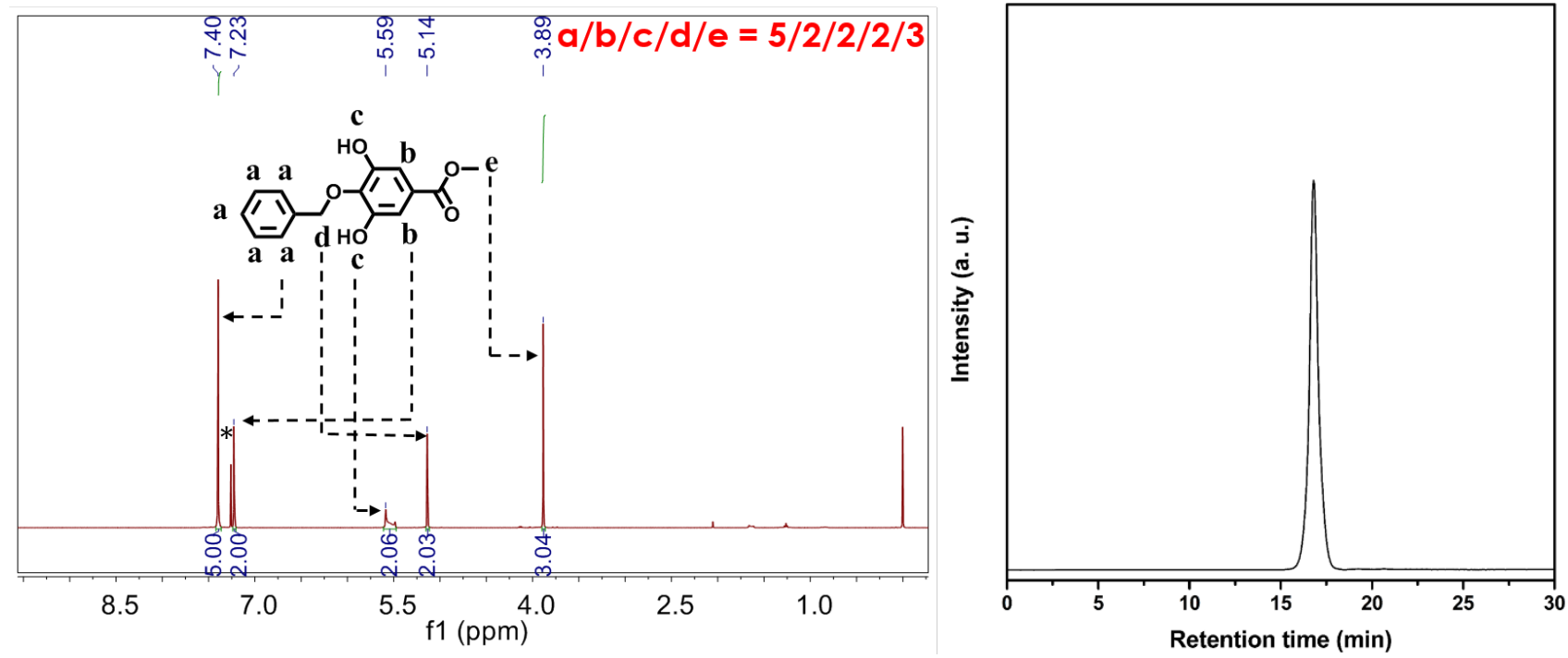

Figure S1. ${ }^{1} \mathrm{H}$ NMR spectra (left) and HPLC trace (right) of 2. The hydroxyl group in the 4-position has been successfully protected by benzyl group in 2 .

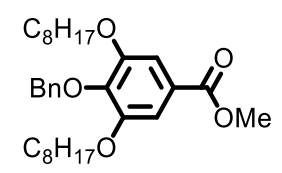

Methyl 4-(benzyloxy)-3,5-bis(octyloxy)benzoate (3-8). Compound 3-8 was synthesized by Williamson etherification under conditions previously reported by our group. ${ }^{4}$ A mixture of $2(4.39 \mathrm{~g}, 16.0 \mathrm{mmol})$ and $\mathrm{K}_{2} \mathrm{CO}_{3}$ (13.27 g, $\left.96.0 \mathrm{mmol}\right)$ was stirred in dry DMF $(80 \mathrm{~mL})$ under $\mathrm{N}_{2}$ protection. 1-Bromooctane (5.8 $\mathrm{mL}, 33.6 \mathrm{mmol}$ ) was added and the reaction mixture was heated at $120{ }^{\circ} \mathrm{C}$ for $3.5 \mathrm{~h}$. Then the mixture was cooled, filtered, poured into cold water $(400 \mathrm{~mL})$ and extracted with EA $(100 \mathrm{~mL} \times 4)$. The combined EA phase was washed with water $(100 \mathrm{~mL} \times 3)$ and brine $(100 \mathrm{~mL})$, dried over anhydrous $\mathrm{MgSO}_{4}$, filtered and evaporated in vacuo. Column chromatography $(\mathrm{EA} / \mathrm{Hexanes}=1 / 24)$ gave methyl 3-8 as a light yellow oil. Product: 7.10 g. Yield: 89\%. Purity, HPLC: 99+\%. TLC (EA/Hexanes =1/9): $R_{f}=0.60$. 
${ }^{1} \mathrm{H}$ NMR (500 MHz, $\left.\mathrm{CDCl}_{3}, \delta\right): 7.53-7.48$ (m, 2H, ArH ortho to $\left.\mathrm{CH}_{2} \mathrm{OAr}\right), 7.37-7.32$ (m, 2H, ArH meta to $\mathrm{CH}_{2} \mathrm{OAr}$ ), 7.31-7.28 (m, 1H, $\mathrm{ArH}$ para to $\mathrm{CH}_{2} \mathrm{OAr}$ ), 7.26 (s, 2H, ArH ortho to $\left.\mathrm{CO}_{2} \mathrm{CH}_{3}\right), 5.08$ (s, 2H, $\left.-\mathrm{OCH}_{2} \mathrm{Ar}\right), 4.01\left(\mathrm{t}, \mathrm{J}=6.5 \mathrm{~Hz}, 4 \mathrm{H},-\mathrm{OCH}_{2} \mathrm{CH}_{2}-\right), 3.89$ (s, 3H, $\left.-\mathrm{CO}_{2} \mathrm{CH}_{3}\right), 1.88-1.75\left(\mathrm{~m}, 4 \mathrm{H},-\mathrm{OCH}_{2} \mathrm{CH}_{2}-\right.$ ), 1.53-1.42 (m, 4H, $\left.-\mathrm{O}\left(\mathrm{CH}_{2}\right)_{2} \mathrm{CH}_{2}-\right), 1.40-1.21\left(\mathrm{~m}, 16 \mathrm{H},-\mathrm{O}\left(\mathrm{CH}_{2}\right)_{3}\left(\mathrm{CH}_{2}\right)_{4}-\right), 0.88(\mathrm{t}, J=6.8 \mathrm{~Hz}, 6 \mathrm{H},-$ $\left.\mathrm{CH}_{2} \mathrm{CH}_{3}\right) .{ }^{13} \mathrm{C} \mathrm{NMR}\left(125 \mathrm{MHz}, \mathrm{CDCl}_{3}, \delta\right): 166.98,153.01,141.86,137.98,128.35,128.26,127.95$, 125.23, 107.94, 74.97, 69.28, 52.22, 31.97, 29.51, 29.46, 29.41, 26.25, 22.82, 14.23. MALDI-TOF MS $m / z$ of $[\mathrm{M}+\mathrm{H}]^{+}$calculated for $\mathrm{C}_{31} \mathrm{H}_{46} \mathrm{O}_{5}$ : 499.3; Found: 500.5 .
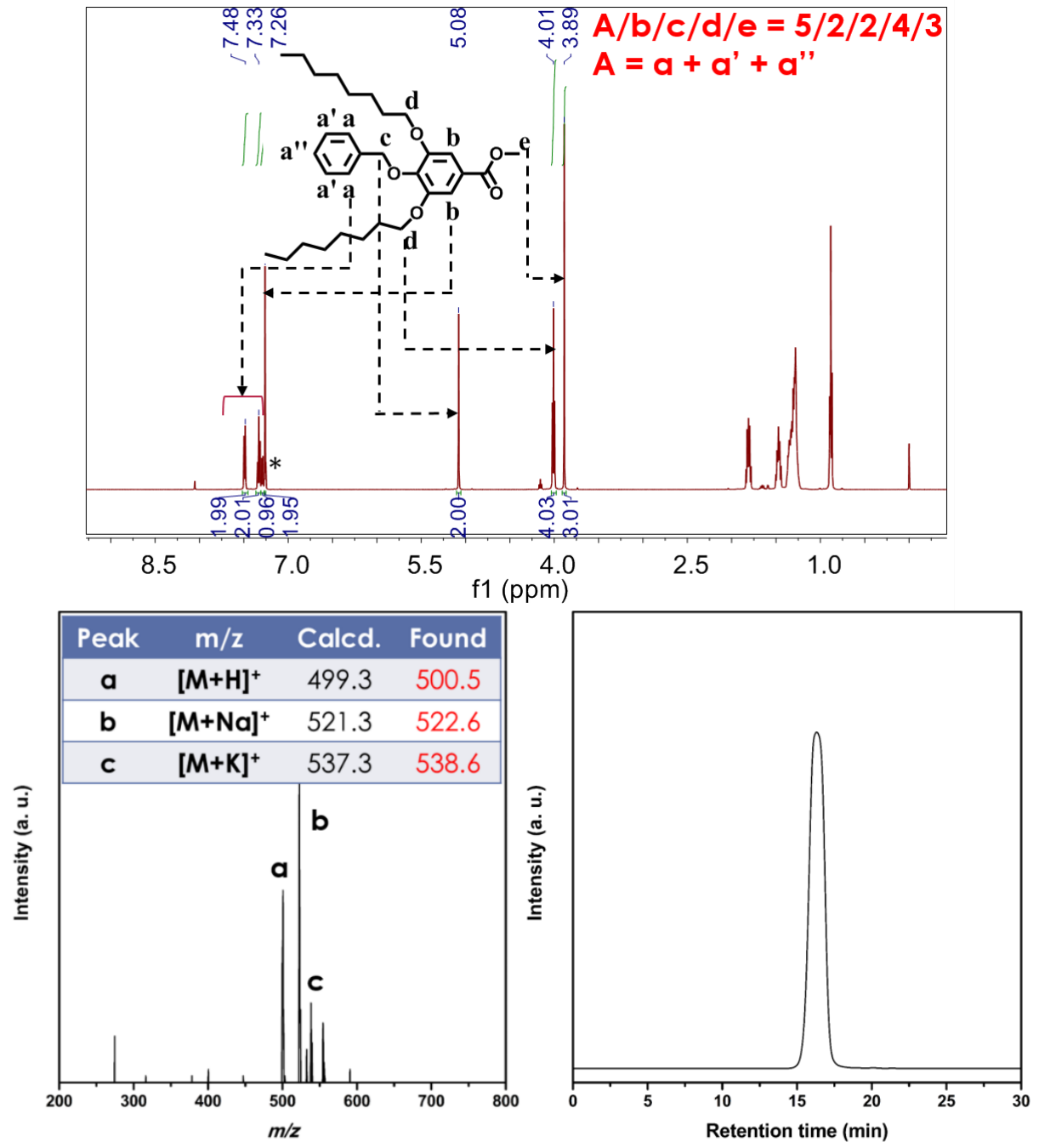

Figure S2. ${ }^{1} \mathrm{H}$ NMR spectra (top), MALDI-TOF MS spectra (bottom left) and HPLC trace (bottom right) of 3-8. Octyl groups have been successfully linked to the 3- and 5-positions of 3-8. 
<smiles>[R]CCCC(C)CCCC(C)C</smiles>

Methyl 4-(benzyloxy)-3,5-bis((3,7-(rac)-dimethyloctyl)oxy)benzoate (3-r). The synthesis procedure for 3-r is similar to that for 3-8. Starting from 2 (1.37 g, $5.0 \mathrm{mmol}), \mathrm{K}_{2} \mathrm{CO}_{3}(4.14 \mathrm{~g}, 30.0 \mathrm{mmol})$ and (rac)1-bromo-3,7-dimethyloctane $(2.2 \mathrm{~mL}, 10.5 \mathrm{mmol})$ in dry DMF $(20 \mathrm{~mL})$, 3-r was obtained as a light yellow oil after column chromatography $($ EA/Hexanes =1/24). Product: 2.41 g. Yield: 87\%. Purity: 99+\%. TLC $($ EA/Hexanes $=1 / 9): \mathrm{R}_{\mathrm{f}}=0.63$.

${ }^{1} \mathrm{H}$ NMR (500 MHz, $\left.\mathrm{CDCl}_{3}, \delta\right): 7.50-7.46$ (m, 2H, ArH ortho to $\mathrm{CH}_{2} \mathrm{OAr}$ ), 7.36-7.31 (m, 2H, ArH meta to $\mathrm{CH}_{2} \mathrm{OAr}$ ), 7.30-7.28 (m, 1H, ArH para to $\mathrm{CH}_{2} \mathrm{OAr}$ ), 7.28 (s, 2H, ArH ortho to $\left.\mathrm{CO}_{2} \mathrm{CH}_{3}\right), 5.07$ (s, $2 \mathrm{H}$, $\left.-\mathrm{OCH}_{2} \mathrm{Ar}\right), 4.10-4.00\left(\mathrm{~m}, 4 \mathrm{H},-\mathrm{OCH}_{2} \mathrm{CH}_{2}-\right), 3.90\left(\mathrm{~s}, 3 \mathrm{H},-\mathrm{CO}_{2} \mathrm{CH}_{3}\right), 1.93-1.82$ \& 1.66-1.57 (m, 4H, $\left.\mathrm{OCH}_{2} \mathrm{CH}_{2}-\right)$, 1.77-1.66 (m, 2H, $\left.-\mathrm{CH}\left(\mathrm{CH}_{3}\right)_{2}\right), 1.56-1.47\left(\mathrm{~m}, 2 \mathrm{H},-\mathrm{CH}\left(\mathrm{CH}_{3}\right) \mathrm{CH}_{2}-\right), 1.40-1.10(\mathrm{~m}, 12 \mathrm{H},-$ $\left.\left(\mathrm{CH}_{2}\right)_{3}-\right), 0.95\left(\mathrm{~d}, J=6.6 \mathrm{~Hz}, 6 \mathrm{H},-\mathrm{CH}_{2} \mathrm{CH}\left(\mathrm{CH}_{3}\right) \mathrm{CH}_{2}-\right), 0.87\left(\mathrm{~d}, J=6.6 \mathrm{~Hz}, 12 \mathrm{H},-\mathrm{CH}\left(\mathrm{CH}_{3}\right)_{2}\right) .{ }^{13} \mathrm{C} \mathrm{NMR}$ (125 MHz, $\left.\mathrm{CDCl}_{3}, \delta\right): 167.05,153.02,137.94,128.40,128.26,127.97,125.24,107.94,74.96,67.60$, $52.27,39.41,37.49,36.47,29.97,28.12,24.86,22.84,22.73,19.73$. MALDI-TOF MS $m / z$ of $[\mathrm{M}+\mathrm{H}]^{+}$ calculated for $\mathrm{C}_{35} \mathrm{H}_{54} \mathrm{O}_{5}$ : 555.4; Found: 556.2 .
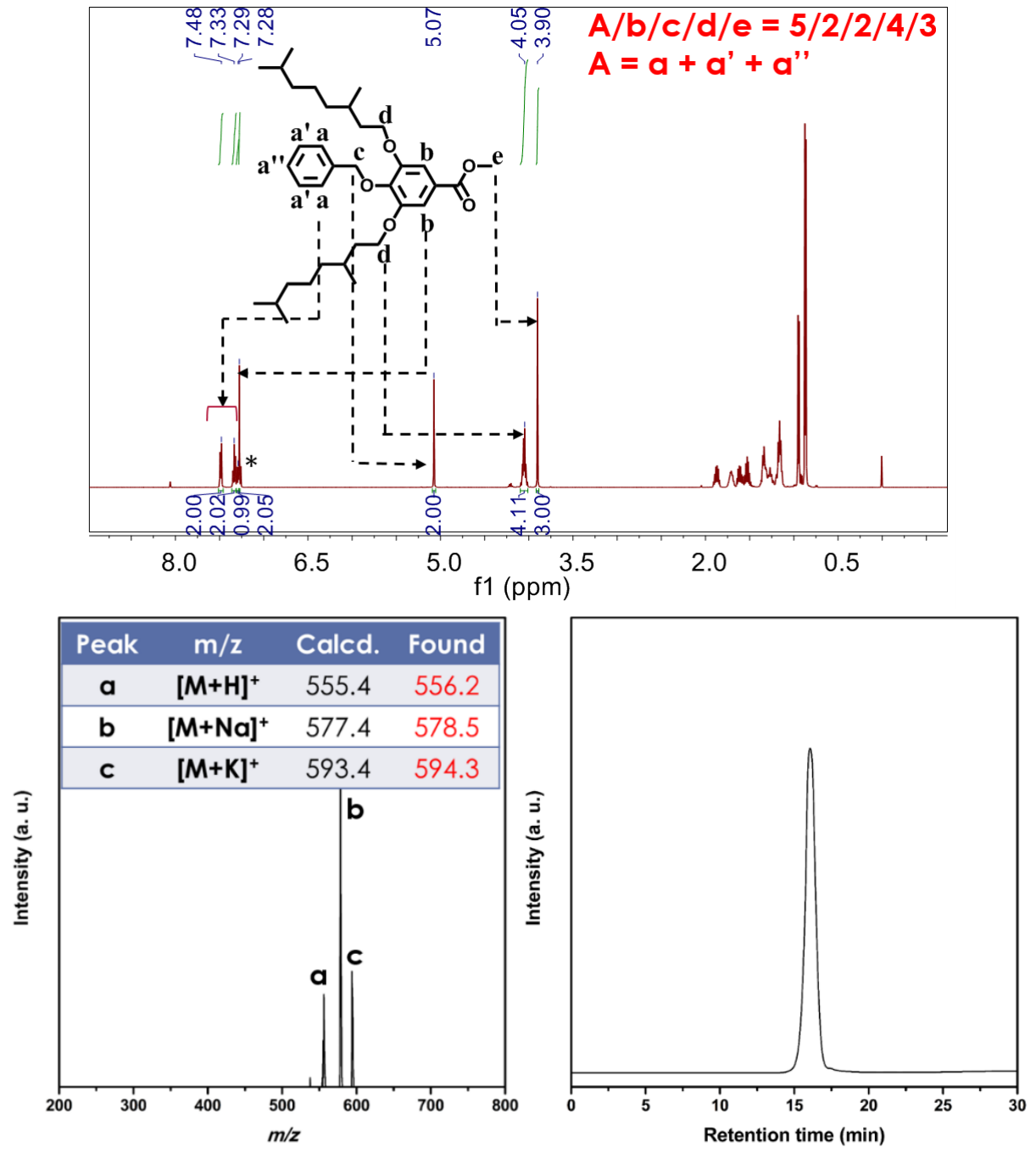

Figure S3. ${ }^{1} \mathrm{H}$ NMR spectra (top), MALDI-TOF MS spectra (bottom left) and HPLC trace (bottom right) of 3-r. Racemic dimethyloctyl groups have been successfully linked to the 3- and 5-positions of 3-r. 


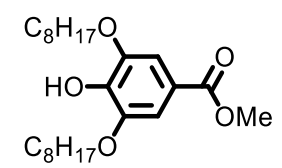

Methyl 4-hydroxy-3,5-bis(octyloxy)benzoate (4-8). Compound 4-8 was synthesized from 3-8 following a procedure previously reported by our group. ${ }^{5}$ Briefly, 3-8 $(2.49 \mathrm{~g}, 5.0 \mathrm{mmol})$, palladium on activated carbon catalyst (140 mg, $\mathrm{Pd} 10 \mathrm{wt} \%), \mathrm{CH}_{2} \mathrm{Cl}_{2}(20 \mathrm{~mL})$ and methanol $(20 \mathrm{~mL})$ were added into a $100 \mathrm{~mL}$ round-bottomed flask, which was sealed with a rubber septum under $\mathrm{N}_{2}$ atmosphere. Then the mixture was purged with $\mathrm{H}_{2}$ for 15 min and allowed to react under $\mathrm{H}_{2}$ atmosphere (balloon) at R. T. After $24 \mathrm{~h}$, the mixture was filtered through celite using $\mathrm{CH}_{2} \mathrm{Cl}_{2}$ as eluent and evacuated to dryness. Finally, the product was dried in a vacuum oven until constant weight was reached. Compound 4-8 was obtained as a white solid. Product: 1.97 g. Yield: 97\%. Purity: 99+\%. TLC (EA/Hexanes $=1 / 9): R_{f}=0.45$. M. P.: 46$47^{\circ} \mathrm{C}$.

${ }^{1} \mathrm{H}$ NMR $\left(500 \mathrm{MHz}, \mathrm{CDCl}_{3}, \delta\right): 7.29$ (s, 2H, $\left.\mathrm{Ar} H\right), 5.88$ (s, $\left.1 \mathrm{H}, \mathrm{ArOH}\right), 4.08$ (t, $J=6.7 \mathrm{~Hz}, 4 \mathrm{H},-\mathrm{OCH}_{2}-$ ), $3.89\left(\mathrm{~s}, 3 \mathrm{H},-\mathrm{CO}_{2} \mathrm{CH}_{3}\right), 1.88-1.78\left(\mathrm{~m}, 4 \mathrm{H},-\mathrm{OCH}_{2} \mathrm{CH}_{2}-\right), 1.50-1.41\left(\mathrm{~m}, 4 \mathrm{H},-\mathrm{O}\left(\mathrm{CH}_{2}\right)_{2} \mathrm{CH}_{2}-\right), 1.40-$ $1.21\left(\mathrm{~m}, 16 \mathrm{H},-\mathrm{O}\left(\mathrm{CH}_{2}\right)_{3}\left(\mathrm{CH}_{2}\right)_{4}-\right), 0.88\left(\mathrm{t}, J=6.9 \mathrm{~Hz}, 6 \mathrm{H},-\mathrm{CH}_{2} \mathrm{CH}_{3}\right) .{ }^{13} \mathrm{C} \mathrm{NMR}\left(125 \mathrm{MHz}, \mathrm{CDCl}_{3}, \delta\right)$ : $167.09,146.23,139.92,121.05,107.85,69.68,52.16,31.94,29.46,29.35,29.33,26.09,22.79,14.23$. MALDI-TOF MS $m / z$ of $[\mathrm{M}+\mathrm{H}]^{+}$calculated for $\mathrm{C}_{24} \mathrm{H}_{40} \mathrm{O}_{5}$ : 409.3; Found: 409.4.
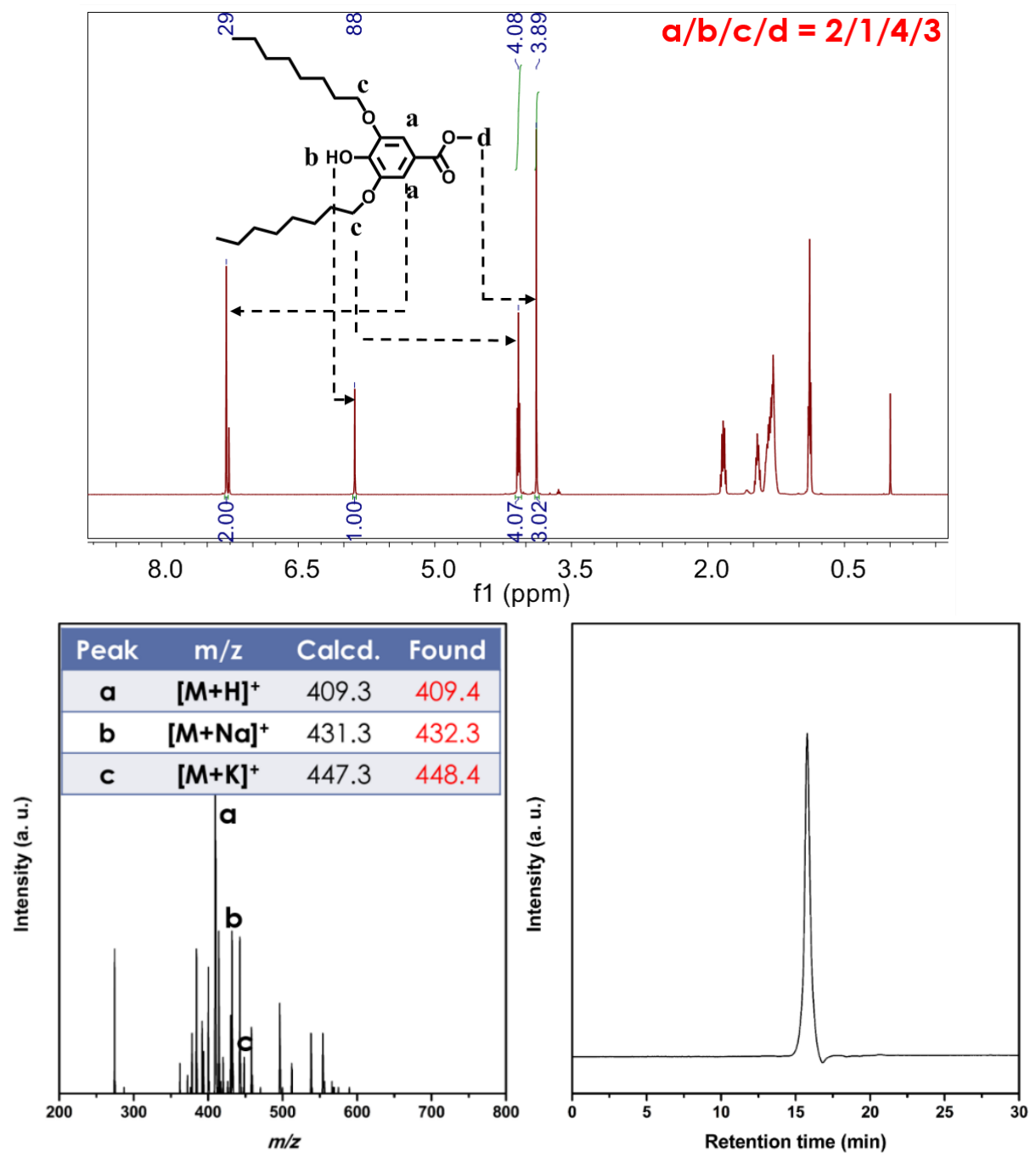

Figure S4. ${ }^{1} \mathrm{H}$ NMR spectra (top), MALDI-TOF MS spectra (bottom left) and HPLC trace (bottom right) of 4-8. The hydroxyl group in the 4-position has been successfully deprotected in 4-8. 


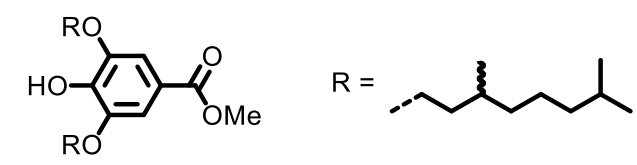

Methyl 4-hydroxy-3,5-bis((3,7-(rac)-dimethyloctyl)oxy)benzoate (4-r). A similar procedure as that for 4-8 was performed to synthesize 4-r. Starting from 3-r $(1.94 \mathrm{~g}, 3.5 \mathrm{mmol})$ and palladium on activated carbon catalyst $(100 \mathrm{mg}, \mathrm{Pd} 10 \mathrm{wt} \%)$ in $\mathrm{CH}_{2} \mathrm{Cl}_{2}(15 \mathrm{~mL}) /$ methanol $(15 \mathrm{~mL})$ mixture, 4-r was obtained as a light orange oil. Product: 1.63 g. Yield: 100\%. Purity by HPLC: $99+\%$. TLC (EA/Hexanes $=1 / 9): R_{f}=$ 0.55 .

${ }^{1} \mathrm{H}$ NMR (500 MHz, $\left.\mathrm{CDCl}_{3}, \delta\right): 7.30$ (s, 2H, ArH), 5.85 (s, 1H, $\left.\mathrm{ArOH}\right), 4.18-4.06\left(\mathrm{~m} \mathrm{4H},-\mathrm{OCH}_{2}-\right), 3.89$ $\left(\mathrm{s}, 3 \mathrm{H},-\mathrm{CO}_{2} \mathrm{CH}_{3}\right), 1.94-1.82\left(\mathrm{~m}, 2 \mathrm{H},-\mathrm{CH}\left(\mathrm{CH}_{3}\right)_{2}\right), 1.72-1.58\left(\mathrm{~m}, 4 \mathrm{H},-\mathrm{OCH}_{2} \mathrm{CH}_{2}-\right), 1.58-1.47(\mathrm{~m}, 2 \mathrm{H}$, $\left.-\mathrm{CH}\left(\mathrm{CH}_{3}\right) \mathrm{CH}_{2}-\right), 1.40-1.08\left(\mathrm{~m}, 12 \mathrm{H},-\left(\mathrm{CH}_{2}\right)_{3}-\right), 0.95\left(\mathrm{~d}, J=6.3 \mathrm{~Hz}, 6 \mathrm{H},-\mathrm{CH}_{2} \mathrm{CH}\left(\mathrm{CH}_{3}\right) \mathrm{CH}_{2}-\right), 0.86(\mathrm{~d}$, $\left.J=6.6 \mathrm{~Hz}, 12 \mathrm{H},-\mathrm{CH}\left(\mathrm{CH}_{3}\right)_{2}\right) .{ }^{13} \mathrm{C} \mathrm{NMR}\left(125 \mathrm{MHz} \mathrm{CDCl}_{3}, \delta\right): 167.06,146.24,139.97,120.97,107.82$, $67.98,52.11,39.33,37.38,36.24,29.99,28.08,24.77,22.80,22.70,19.79$. MALDI-TOF MS $\mathrm{m} / z$ of $[\mathrm{M}+\mathrm{H}]^{+}$calculated for $\mathrm{C}_{28} \mathrm{H}_{48} \mathrm{O}_{5}$ : 465.4; Found: 465.9 .
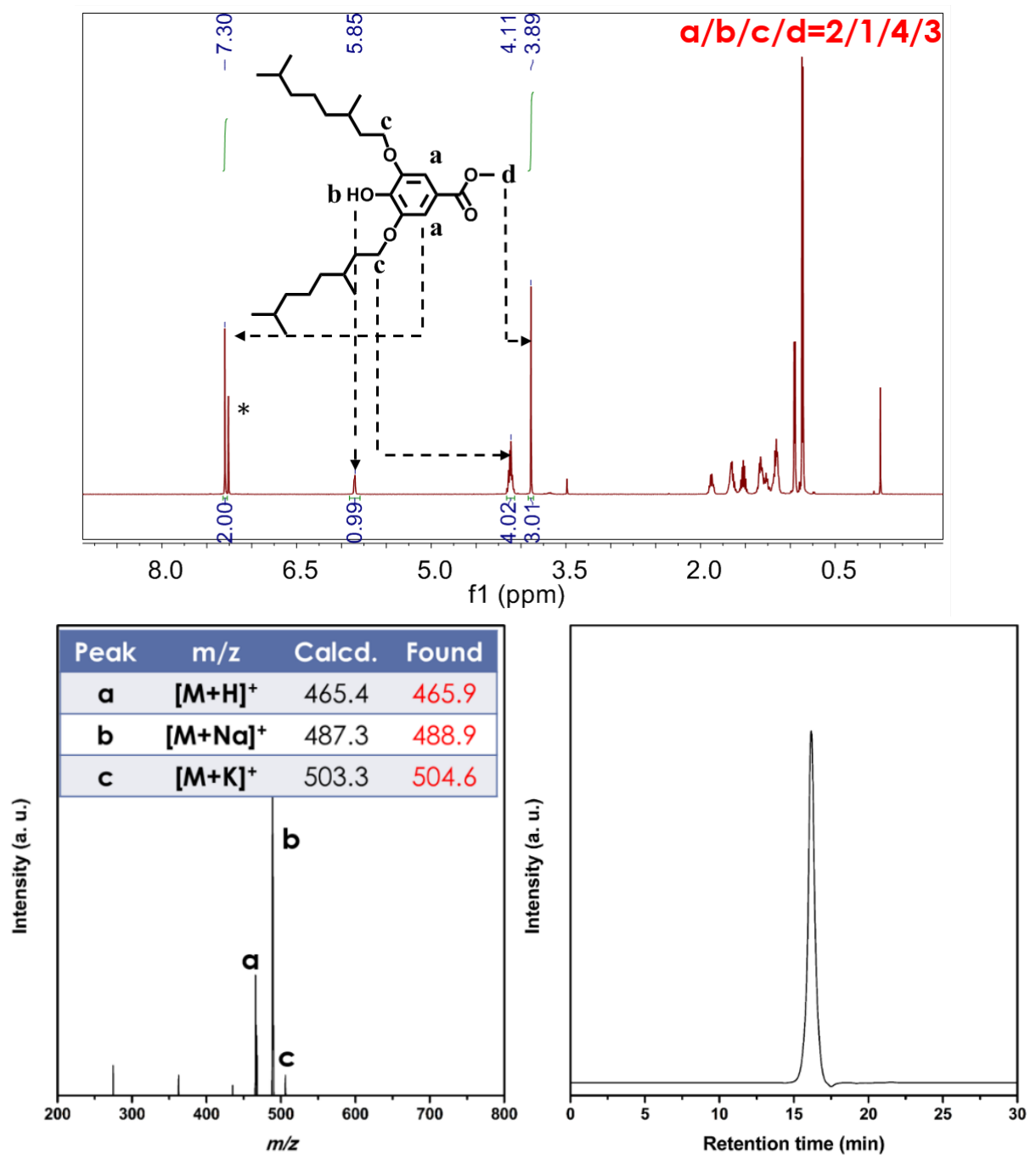

Figure S5. ${ }^{1} \mathrm{H}$ NMR spectra (top), MALDI-TOF MS spectra (bottom left) and HPLC trace (bottom right) of 4-r. The hydroxyl group in the 4-position has been successfully deprotected in 4-r. 


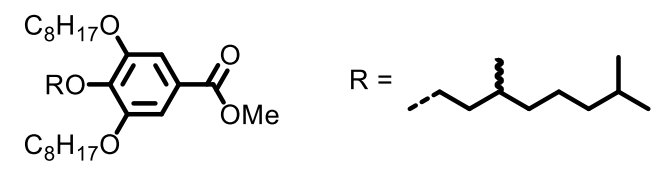

Methyl 4-(((rac)-3,7-dimethyloctyl)oxy)-3,5-bis(octyloxy)benzoate (5-8r8). Following a procedure similar to that for 3-8, reaction of 4-8 $(1.23 \mathrm{~g}, 3.0 \mathrm{mmol})$ and ( $\mathrm{rac})$-1-bromo-3,7-dimethyloctane $(0.65$ $\mathrm{mL}, 3.2 \mathrm{mmol})$ in the presence of $\mathrm{K}_{2} \mathrm{CO}_{3}(2.49 \mathrm{~g}, 18.0 \mathrm{mmol})$ gave 5-8r8 as a light yellow oil. Product: 1.67 g. Yield: $100 \%$. Purity by HPLC: $99+\%$. TLC $(\mathrm{EA} /$ Hexanes $=1 / 9): \mathrm{R}_{\mathrm{f}}=0.64$.

${ }^{1} \mathrm{H}$ NMR (500 MHz, $\left.\mathrm{CDCl}_{3}, \delta\right): 7.25(\mathrm{~s}, 2 \mathrm{H}, \mathrm{ArH}), 4.11-4.03\left(\mathrm{~m}, 2 \mathrm{H},-\mathrm{OCH}_{2} \mathrm{CH}_{2} \mathrm{CH}\left(\mathrm{CH}_{3}\right)-\right), 4.01(\mathrm{t}, J$ $\left.=6.5 \mathrm{~Hz}, 4 \mathrm{H},-\mathrm{OCH}_{2} \mathrm{CH}_{2} \mathrm{CH}_{2}-\right), 3.89\left(\mathrm{~s}, 3 \mathrm{H},-\mathrm{CO}_{2} \mathrm{CH}_{3}\right), 1.88-1.76\left(\mathrm{~m}, 5 \mathrm{H},-\mathrm{CH}_{\left(\mathrm{CH}_{3}\right)_{2} \text { and }-}\right.$

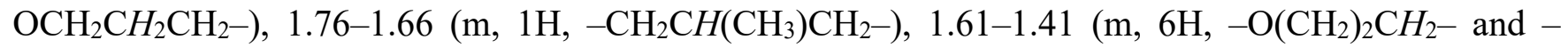
$\left.\mathrm{OCH}_{2} \mathrm{CH}_{2} \mathrm{CH}\left(\mathrm{CH}_{3}\right)^{-}\right), 1.42-1.08\left(\mathrm{~m}, 22 \mathrm{H},-\mathrm{O}\left(\mathrm{CH}_{2}\right)_{3}\left(\mathrm{CH}_{2}\right)_{4}-\right.$ and $\left.-\left(\mathrm{CH}_{2}\right)_{3} \mathrm{CH}\left(\mathrm{CH}_{3}\right)_{2}\right), 0.92(\mathrm{~d}, J=6.5 \mathrm{~Hz}$, $\left.3 \mathrm{H},-\mathrm{CH}_{2} \mathrm{CH}\left(\mathrm{CH}_{3}\right) \mathrm{CH}_{2}-\right), 0.91-0.82\left(\mathrm{~m}, 12 \mathrm{H},-\mathrm{CH}\left(\mathrm{CH}_{3}\right)_{2}\right.$ and $\left.-\mathrm{CH}_{2} \mathrm{CH}_{3}\right) .{ }^{13} \mathrm{C} \mathrm{NMR}\left(125 \mathrm{MHz}, \mathrm{CDCl}_{3}\right.$,

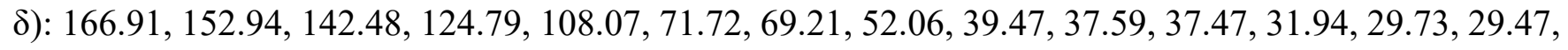
29.44, 29.40, 28.10, 26.21, 24.84, 22.77, 22.68, 19.63, 14.17. MALDI-TOF MS $m / z$ of $[\mathrm{M}+\mathrm{H}]^{+}$ calculated for $\mathrm{C}_{34} \mathrm{H}_{60} \mathrm{O}_{5}$ : 549.5; Found: 549.7 .
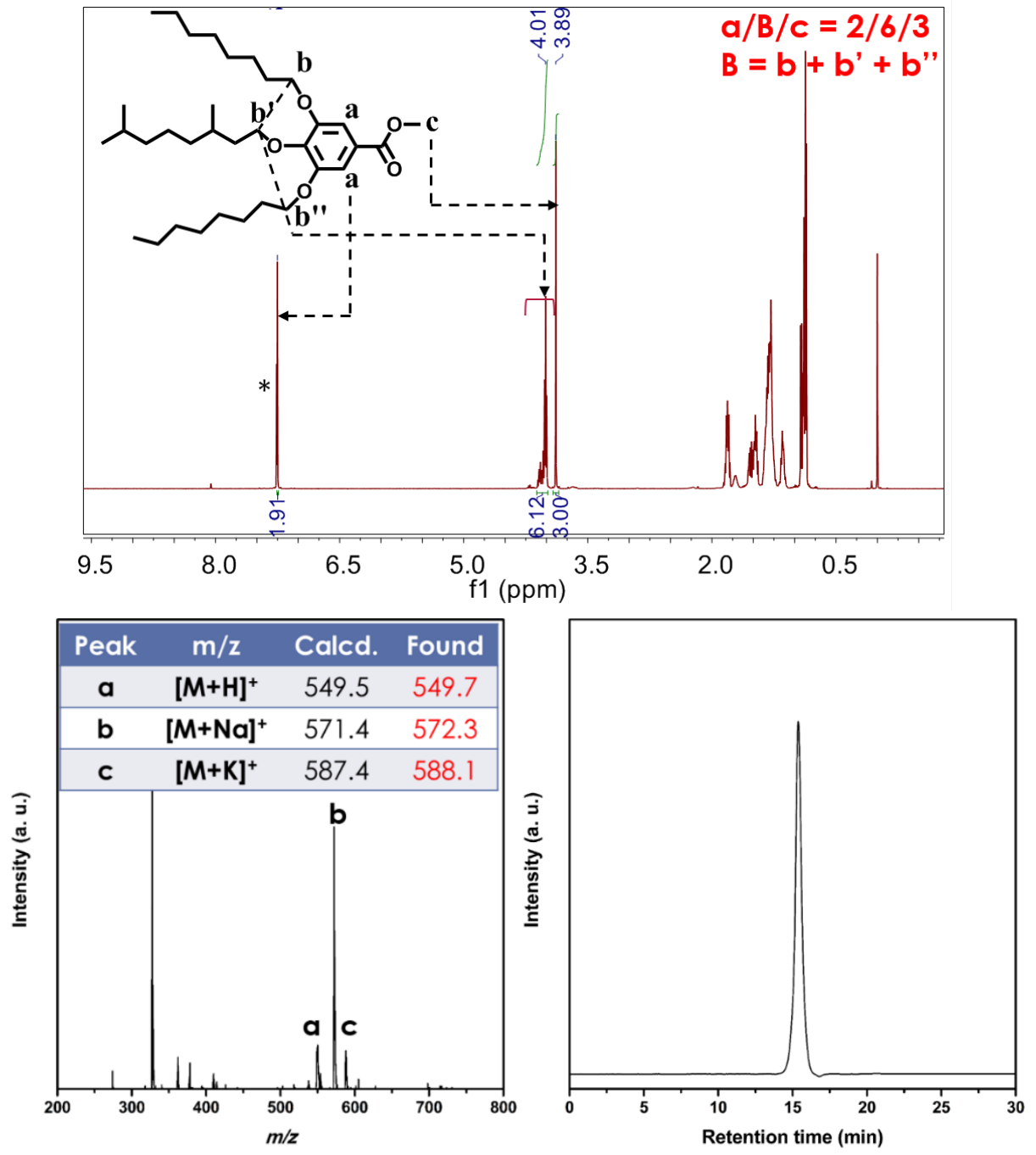

Figure S6. ${ }^{1} \mathrm{H}$ NMR spectra (top), MALDI-TOF MS spectra (bottom left) and HPLC trace (bottom right) of 5-8r8. Racemic dimethyloctyl group has been successfully linked to the 4-position of 5-8r8. 
COMe<smiles>[R][CH]CC[C@H](C)CCCC(C)C</smiles>

Methyl 3,5-bis(((rac)-3,7-dimethyloctyl)oxy)-4-(octyloxy)benzoate (5-r8r). The synthesis procedure for 5-r8r is similar to that for 3-8. Starting from 4-r (1.39 g, $3.0 \mathrm{mmol}), \mathrm{K}_{2} \mathrm{CO}_{3}(2.49 \mathrm{~g}, 18.0 \mathrm{mmol})$ and 1-bromooctane $(0.55 \mathrm{~mL}, 3.2 \mathrm{mmol})$ in dry DMF $(20 \mathrm{~mL})$, Compound 5-r8r was obtained as a light yellow oil. Product: 1.72 g. Yield: 100\%. Purity by HPLC: 99+\%. TLC (EA/Hexanes =1/9): $\mathrm{R}_{\mathrm{f}}=0.65$.

${ }^{1} \mathrm{H}$ NMR (500 MHz, $\left.\mathrm{CDCl}_{3}, \delta\right): 7.26$ (s, 2H, ArH), 4.09-3.96 (m, 6H, $\left.-\mathrm{OCH}_{2}-\right), 3.89\left(\mathrm{~s}, 3 \mathrm{H},-\mathrm{CO}_{2} \mathrm{CH}_{3}\right)$, 1.92-1.81 (m, 2H, $\left.-\mathrm{CH}\left(\mathrm{CH}_{3}\right)_{2}\right), 1.78-1.41\left(\mathrm{~m}, 10 \mathrm{H},-\mathrm{OCH}_{2} \mathrm{CH}_{2} \mathrm{CH}\left(\mathrm{CH}_{3}\right)-,-\mathrm{OCH}_{2} \mathrm{CH}_{2} \mathrm{CH}_{2}-\right.$, $\mathrm{CH}_{2} \mathrm{CH}\left(\mathrm{CH}_{3}\right) \mathrm{CH}_{2}-$ and $\left.-\mathrm{OCH}_{2} \mathrm{CH}_{2} \mathrm{CH}_{2}-\right), 1.40-1.08\left(\mathrm{~m}, 20 \mathrm{H},-\mathrm{CH}\left(\mathrm{CH}_{3}\right)\left(\mathrm{CH}_{2}\right)_{3}-\right.$ and $-\mathrm{O}\left(\mathrm{CH}_{2}\right)_{3}\left(\mathrm{CH}_{2}\right)_{4}-$ ), $0.94\left(\mathrm{~d}, J=6.6 \mathrm{~Hz}, 6 \mathrm{H}, \mathrm{CH}_{2} \mathrm{CH}\left(\mathrm{CH}_{3}\right) \mathrm{CH}_{2}-\right), 0.91-0.81\left(\mathrm{~m}, 15 \mathrm{H},-\mathrm{CH}\left(\mathrm{CH}_{3}\right)_{2}\right.$ and $\left.-\mathrm{CH}_{2} \mathrm{CH}_{3}\right) .{ }^{13} \mathrm{C} \mathrm{NMR}$ (125 MHz, $\left.\mathrm{CDCl}_{3}, \delta\right):$ 167.08, 153.00, 142.54, 124.84, 108.13, 73.60, 67.62, 52.23, 39.43, 37.49, 36.50, $32.08,30.52$, 29.99, 29.69, 29.54, 28.16, 26.24, 24.91, 22.87, 22.76, 19.78, 14.27. MALDI-TOF MS m/z of $[\mathrm{M}+\mathrm{H}]^{+}$calculated for $\mathrm{C}_{36} \mathrm{H}_{64} \mathrm{O}_{5}$ : 577.5; Found: 577.9 .
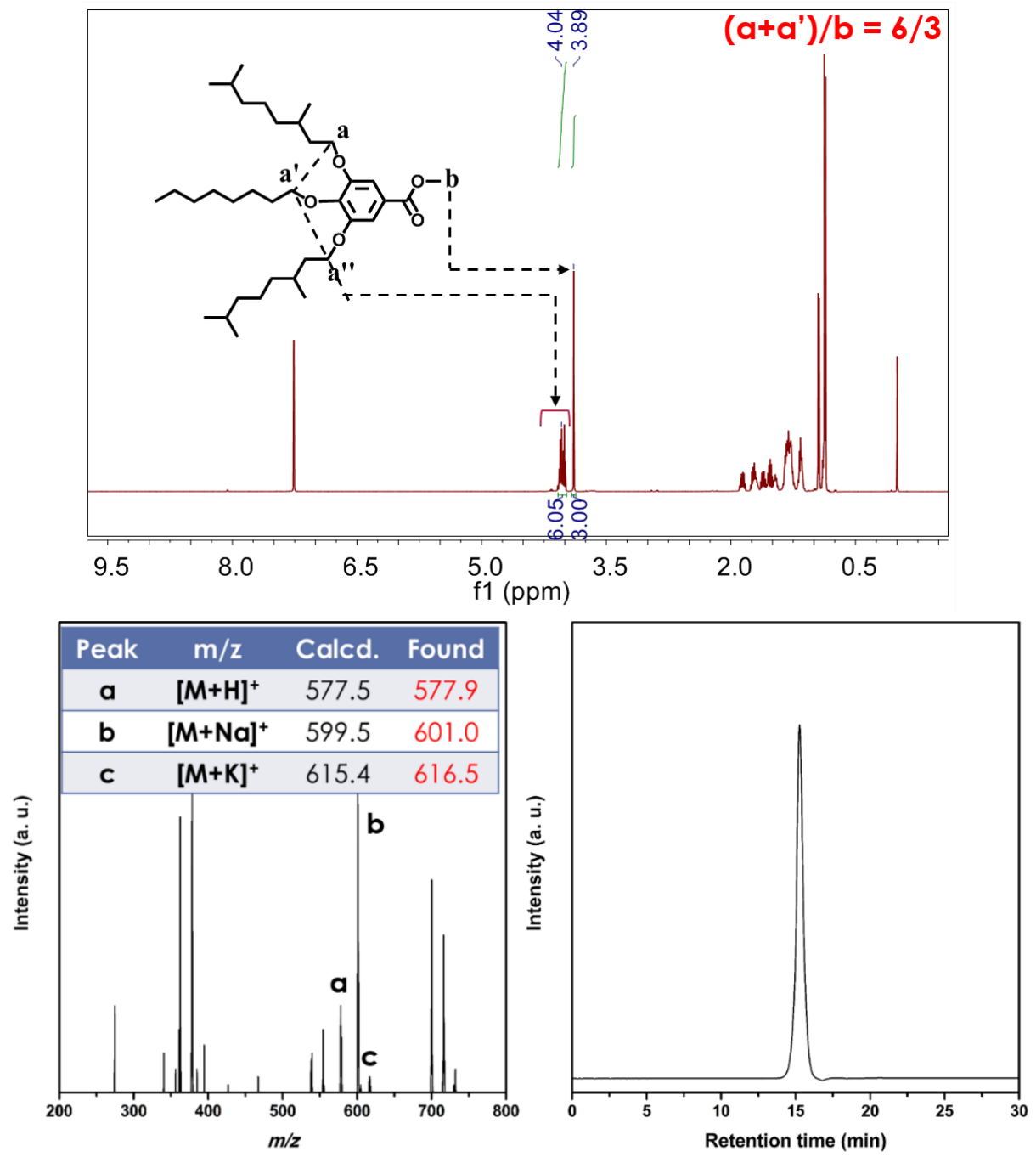

Figure S7. ${ }^{1} \mathrm{H}$ NMR spectra (top), MALDI-TOF MS spectra (bottom left) and HPLC trace (bottom right) of 5-r8r. Octyl group has been successfully linked to the 4-position of 5-r8r. 


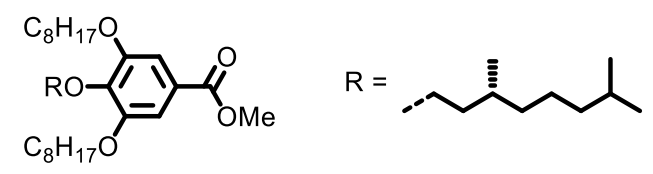

Methyl 4-(((3S)-3,7-dimethyloctyl)oxy)-3,5-bis(octyloxy)benzoate (5-8S8). Compound 5-8S8 was synthesized following a procedure similar to that for 3-8. From 4-8 (1.23 g, $3.0 \mathrm{mmol})$ and (3S)-1-bromo3,7-dimethyloctane $(0.65 \mathrm{~mL}, 3.2 \mathrm{mmol})$ in the presence of $\mathrm{K}_{2} \mathrm{CO}_{3}(2.49 \mathrm{~g}, 18.0 \mathrm{mmol})$ in dry DMF (20 mL), 5-8S8 was obtained as a light yellow oil. Product: $1.58 \mathrm{~g}$. Yield: 96\%. Purity by HPLC: 99+\%. TLC $(\mathrm{EA} /$ Hexanes $=1 / 9): \mathrm{R}_{\mathrm{f}}=0.64$.

${ }^{1} \mathrm{H}$ NMR (400 MHz, $\left.\mathrm{CDCl}_{3}, \delta\right): 7.25$ (s, 2H, $\left.\mathrm{ArH}\right), 4.13-4.03\left(\mathrm{~m}, 2 \mathrm{H},-\mathrm{OCH}_{2} \mathrm{CH}_{2} \mathrm{CH}\left(\mathrm{CH}_{3}\right)-\right), 4.01(\mathrm{t}, J=$

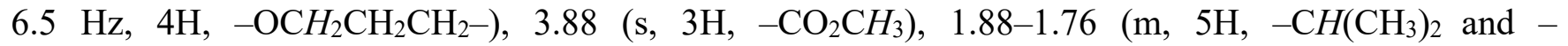

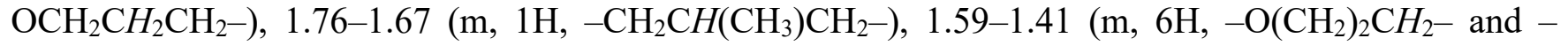
$\left.\mathrm{OCH}_{2} \mathrm{CH}_{2} \mathrm{CH}\left(\mathrm{CH}_{3}\right)-\right), 1.41-1.08\left(\mathrm{~m}, 22 \mathrm{H},-\mathrm{O}\left(\mathrm{CH}_{2}\right)_{3}\left(\mathrm{CH}_{2}\right)_{4}-\right.$ and $\left.-\left(\mathrm{CH}_{2}\right)_{3} \mathrm{CH}\left(\mathrm{CH}_{3}\right)_{2}\right), 0.92(\mathrm{~d}, J=6.6 \mathrm{~Hz}$, $\left.3 \mathrm{H},-\mathrm{CH}_{2} \mathrm{CH}\left(\mathrm{CH}_{3}\right) \mathrm{CH}_{2}-\right), 0.91-0.81\left(\mathrm{~m}, 12 \mathrm{H},-\mathrm{CH}\left(\mathrm{CH}_{3}\right)_{2}\right.$ and $\left.-\mathrm{CH}_{2} \mathrm{CH}_{3}\right) .{ }^{13} \mathrm{C} \mathrm{NMR}\left(100 \mathrm{MHz}, \mathrm{CDCl}_{3}\right.$,

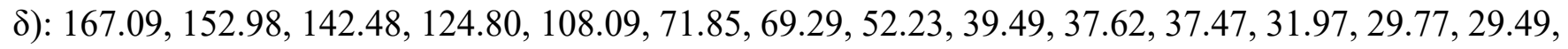
29.44, 29.42, 28.13, 26.22, 24.86, 22.83, 22.81, 22.74, 19.67, 14.23. MALDI-TOF MS $m / z$ of $[\mathrm{M}+\mathrm{H}]^{+}$ calculated for $\mathrm{C}_{34} \mathrm{H}_{60} \mathrm{O}_{5}$ : 549.5; Found: 550.4 .
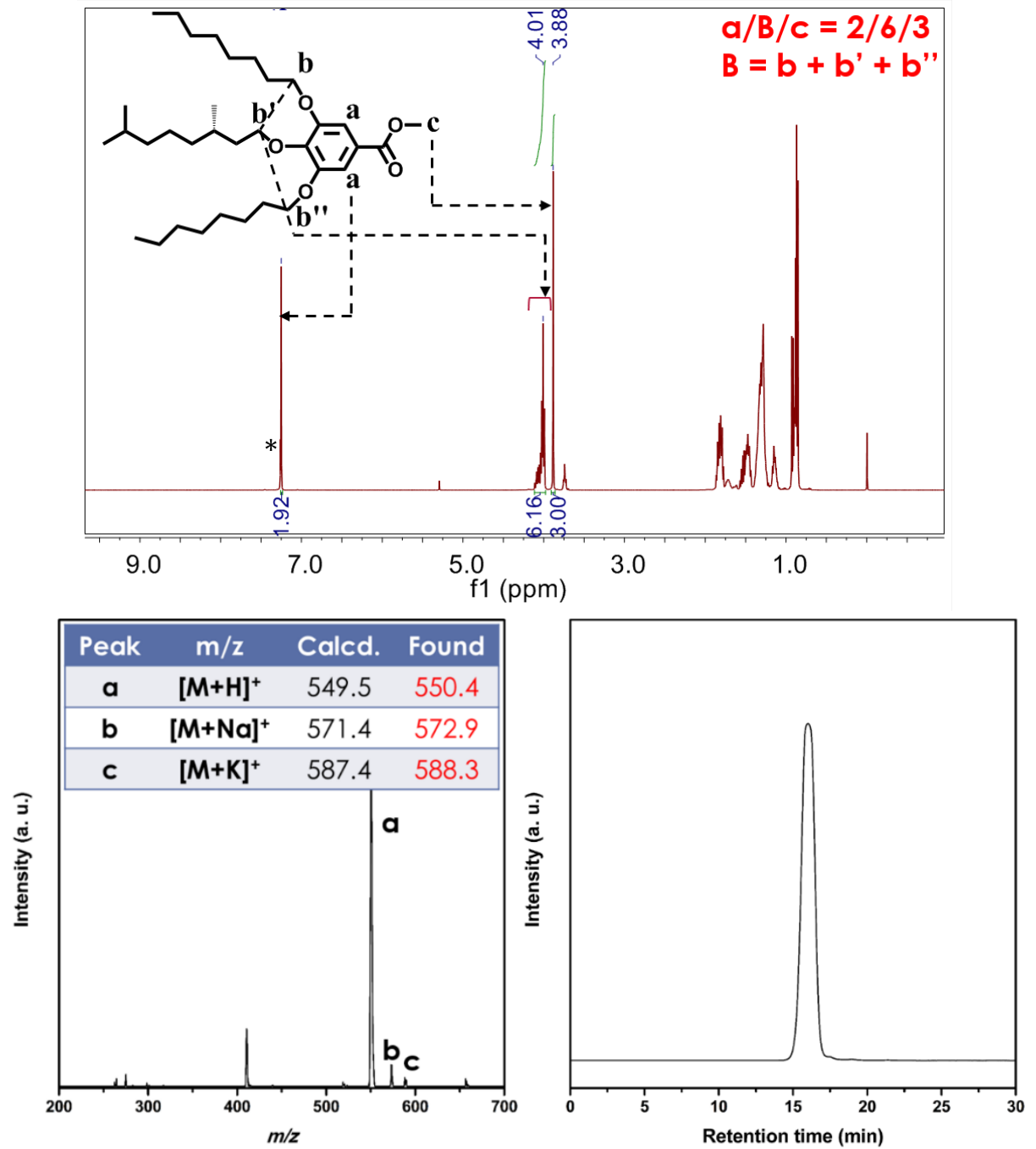

Figure S8. ${ }^{1} \mathrm{H}$ NMR spectra (top), MALDI-TOF MS spectra (bottom left) and HPLC trace (bottom right) of 5-8S8. Chiral dimethyloctyl group has been successfully linked to the 4-position of 5-8S8. 


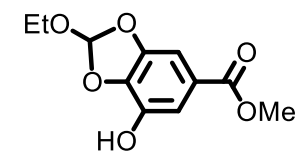

Methyl 2-ethoxy-7-hydroxybenzo $[\boldsymbol{d}][1,3]$ dioxole-5-carboxylate (6). Compound 6 was synthesized from 1 using a method previously reported by our group. ${ }^{5}$ A mixture of 1 (10.00 g, $\left.54.3 \mathrm{mmol}\right)$, triethyl orthoformate $(27.1 \mathrm{~mL}, 163 \mathrm{mmol})$ and Amberlyst-15(H) $(0.27 \mathrm{~g})$ was refluxed in toluene $(250 \mathrm{~mL})$ for $18 \mathrm{~h}$ under $\mathrm{N}_{2}$ protection. EtOH/toluene was removed azeotropically using a Dean-Stark trap. Then the reaction mixture was cooled, filtered through celite and evaporated to dryness. The product was subjected to column chromatography with EA/hexanes $(15 / 85)$ as eluent and $\mathbf{6}$ was obtained as white powder. Product: 11.41 g. Yield: 88\%. Purity by HPLC: 99+\%. TLC (EA/Hexanes = 1/2): $\mathrm{R}_{\mathrm{f}}=0.53$. M. P.: 96-97 ${ }^{\circ} \mathrm{C}$.

${ }^{1} \mathrm{H}$ NMR (500 MHz, $\left.\mathrm{CDCl}_{3}, \delta\right): 7.41$ (d, $J=1.5 \mathrm{~Hz}, 1 \mathrm{H}, \operatorname{ArH}$ ortho to $\left.-\mathrm{OH}\right), 7.18(\mathrm{~d}, J=1.5 \mathrm{~Hz}, 1 \mathrm{H}, \operatorname{ArH}$ para to $-\mathrm{OH}), 6.95(\mathrm{~s}, 1 \mathrm{H},-\mathrm{C}(H) \mathrm{OEt}), 5,73(\mathrm{~s}, 1 \mathrm{H},-\mathrm{OH}), 3.89\left(\mathrm{~s}, 3 \mathrm{H},-\mathrm{OCH}_{3}\right), 3.80-3.72(\mathrm{~m}, 2 \mathrm{H},-$ $\left.\mathrm{OCH}_{2} \mathrm{CH}_{3}\right), 1.27\left(\mathrm{t}, J=7.1 \mathrm{~Hz}, 3 \mathrm{H},-\mathrm{OCH}_{2} \mathrm{CH}_{3}\right) .{ }^{13} \mathrm{C} \mathrm{NMR}\left(125 \mathrm{MHz}, \mathrm{CDCl}_{3}, \delta\right)$ : 166.36, 147.23, 144.56, $139.70,124.18,119.83,113.84,109.34,59.44,51.91,14.72$.
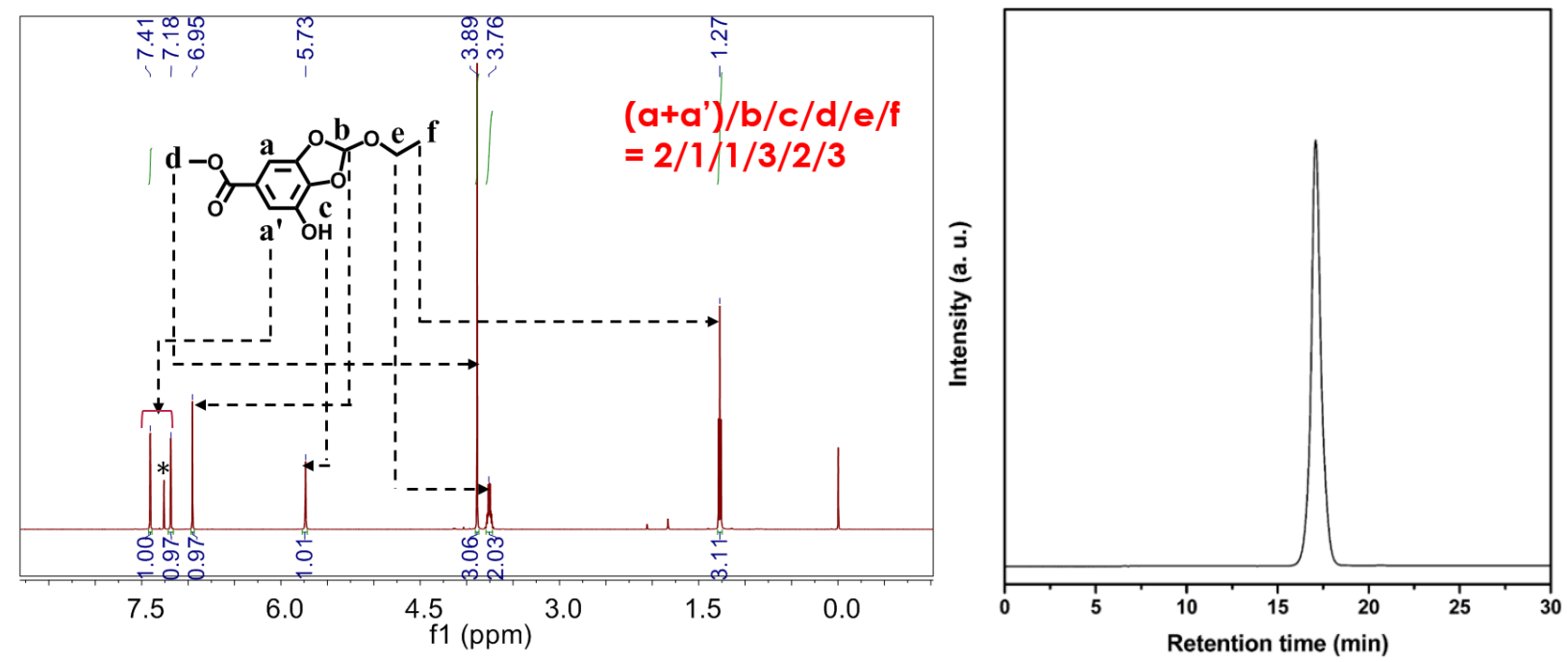

Figure S9. ${ }^{1} \mathrm{H}$ NMR spectra (left) and HPLC trace (right) of 6 . The hydroxyl groups in the 3- and 4positions have been successfully protected by triethyl orthoformate in 6 . 


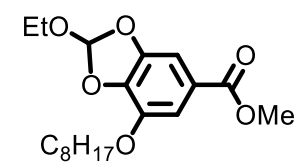

Methyl 2-ethoxy-7-(octyloxy)benzo $[d][1,3]$ dioxole-5-carboxylate (7-8). Compound $7-8$ was synthesized by Williamson etherification under conditions reported by our group. ${ }^{6}$ A mixture of 6 (2.40 $\mathrm{g}, 10.0 \mathrm{mmol}$ ) and $\mathrm{K}_{2} \mathrm{CO}_{3}(2.76 \mathrm{~g}, 20.0 \mathrm{mmol})$ was stirred in dry DMF (40 mL) under $\mathrm{N}_{2}$ protection. 1Bromooctane $(1.8 \mathrm{~mL}, 10.5 \mathrm{mmol})$ was added and the reaction mixture was heated at $65{ }^{\circ} \mathrm{C}$ for $4 \mathrm{~h}$. Then the mixture was cooled, filtered, poured into cold water $(200 \mathrm{~mL})$ and extracted with EA $(50 \mathrm{~mL} \times 4)$. The combined EA phase was washed with water $(50 \mathrm{~mL} \times 3)$, dried over anhydrous $\mathrm{MgSO}_{4}$, filtered and evaporated in vacuo. The crude product was subjected to column chromatography $(\mathrm{EA} / \mathrm{Hexanes}=1 / 19)$ and 7-8 was obtained as a yellow oil. Product: 3.54 g. Yield: 100\%. Purity, HPLC: 99+\%. TLC $(\mathrm{EA} /$ Hexanes $=1 / 9): \mathrm{R}_{\mathrm{f}}=0.55$.

${ }^{1} \mathrm{H}$ NMR $\left(500 \mathrm{MHz}, \mathrm{CDCl}_{3}, \delta\right): 7.33\left(\mathrm{~d}, J=1.4 \mathrm{~Hz}, 1 \mathrm{H}, \mathrm{ArH}\right.$ ortho to $\left.-\mathrm{OC}_{8} \mathrm{H}_{17}\right), 7.23(\mathrm{~d}, J=1.4 \mathrm{~Hz}, 1 \mathrm{H}$, ArH para to $\left.-\mathrm{OC}_{8} \mathrm{H}_{17}\right), 6.94(\mathrm{~s}, 1 \mathrm{H},-\mathrm{C}(H) \mathrm{OEt}), 4.11\left(\mathrm{t}, J=6.7 \mathrm{~Hz}, 2 \mathrm{H},-\mathrm{OCH}_{2} \mathrm{CH}_{2}-\right), 3.88(\mathrm{~s}, 3 \mathrm{H},-$ $\left.\mathrm{OCH}_{3}\right), 3.79-3.71\left(\mathrm{~m}, 2 \mathrm{H},-\mathrm{OCH}_{2} \mathrm{CH}_{3}\right), 1.86-1.76\left(\mathrm{~m}, 2 \mathrm{H},-\mathrm{OCH}_{2} \mathrm{CH}_{2}-\right), 1.50-1.41(\mathrm{~m}, 2 \mathrm{H},-$ $\left.\mathrm{O}\left(\mathrm{CH}_{2}\right)_{2} \mathrm{CH}_{2}-\right), 1.39-1.23\left(\mathrm{~m}, 11 \mathrm{H},-\left(\mathrm{CH}_{2}\right)_{3}\left(\mathrm{CH}_{2}\right)_{4}-\right.$ and $\left.-\mathrm{OCH}_{2} \mathrm{CH}_{3}\right), 0.89\left(\mathrm{t}, J=6.9 \mathrm{~Hz}, 3 \mathrm{H},-\mathrm{CH}_{3}\right) .{ }^{13} \mathrm{C}$ $\mathrm{NMR}\left(125 \mathrm{MHz}, \mathrm{CDCl}_{3}, \delta\right): 166.60,147.21,142.32,138.29,124.36,120.12,111.25,103.37,69.86$, 59.72, 52.30, 31.95, 29.46, 29.37, 26.02, 22.80, 14.94, 14.23.
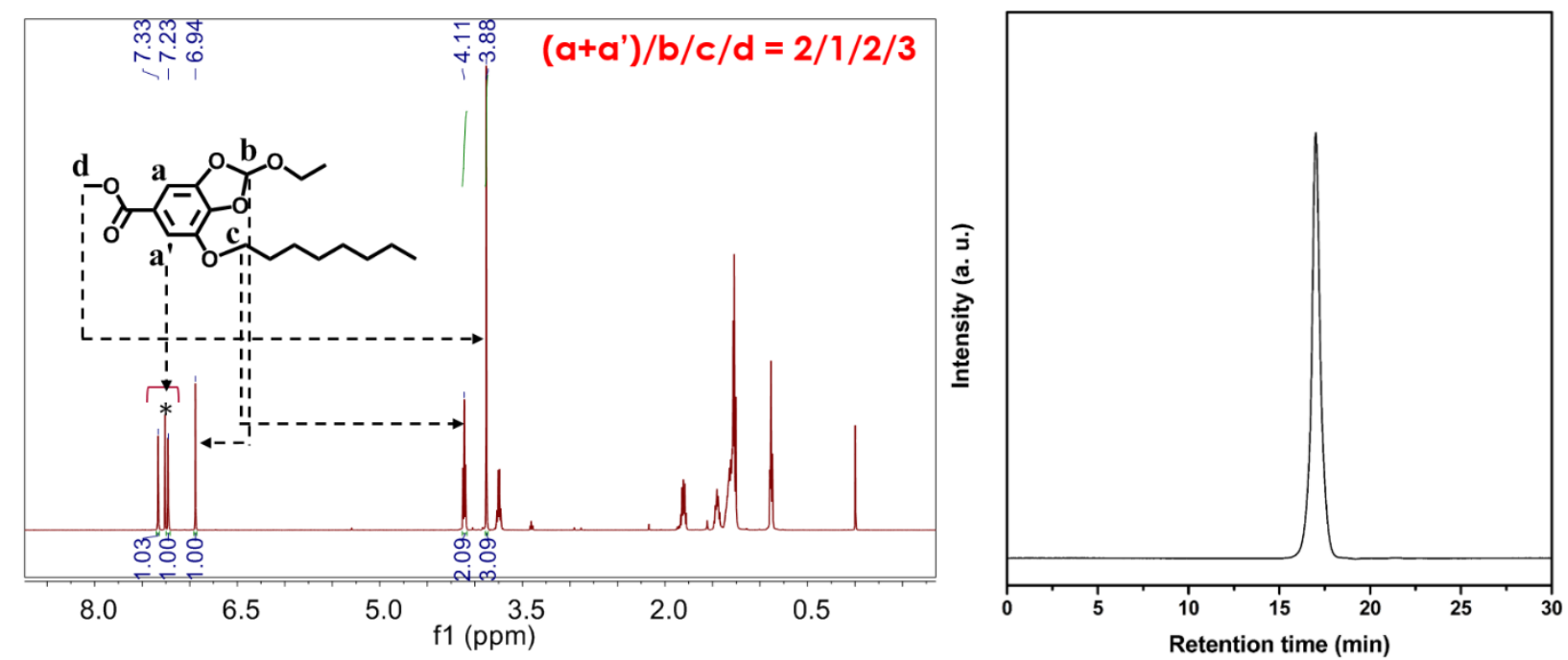

Figure S10. ${ }^{1} \mathrm{H}$ NMR spectra (left) and HPLC trace (right) of 7-8. Octyl group has been successfully linked to the 5-position of 7-8. 


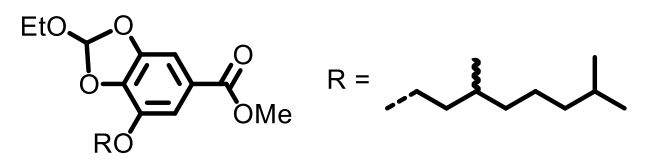

Methyl 7-(((rac)-3,7-dimethyloctyl)oxy)-2-ethoxybenzo[][1,3]dioxole-5-carboxylate (7-r). The synthesis procedure for 7-r is similar to that for 7-8. Starting from 6 (2.40 g, $10.0 \mathrm{mmol})$ and ( $\mathrm{rac})$-1bromo-3,7-dimethyloctane $(2.2 \mathrm{~mL}, 10.5 \mathrm{mmol})$ in the presence of $\mathrm{K}_{2} \mathrm{CO}_{3}(2.76 \mathrm{~g}, 20.0 \mathrm{mmol})$ in dry DMF (40 mL), 7-r was obtained as a yellow oil. Product: 3.73 g. Yield: 98\%. Purity by HPLC: 99+\%. TLC $($ EA/Hexanes $=1 / 9): \mathrm{R}_{\mathrm{f}}=0.60$.

${ }^{1} \mathrm{H} \mathrm{NMR}\left(500 \mathrm{MHz}, \mathrm{CDCl}_{3}, \delta\right): 7.34(\mathrm{~d}, J=1.4 \mathrm{~Hz}, 1 \mathrm{H}, \mathrm{ArH}$ ortho to para to $-\mathrm{OR}), 7.23(\mathrm{~d}, J=1.4 \mathrm{~Hz}$, 1H, ArH para to -OR), 6.94 (s, 1H, $-\mathrm{C}(\mathrm{H}) \mathrm{OEt}), 4.20-4.09\left(\mathrm{~m}, 2 \mathrm{H},-\mathrm{OCH}_{2} \mathrm{CH}_{2}-\right), 3.88\left(\mathrm{~s}, 3 \mathrm{H},-\mathrm{OCH}_{3}\right)$, $3.75\left(\mathrm{~m}, 2 \mathrm{H},-\mathrm{OCH}_{2} \mathrm{CH}_{3}\right), 1.90-1.80\left(\mathrm{~m}, 1 \mathrm{H},-\mathrm{CH}\left(\mathrm{CH}_{3}\right)_{2}\right), 1.73-1.57\left(\mathrm{~m}, 2 \mathrm{H},-\mathrm{OCH}_{2} \mathrm{CH}_{2}-\right), 1.57-1.47$ $\left(\mathrm{m}, 1 \mathrm{H},-\mathrm{CH}\left(\mathrm{CH}_{3}\right) \mathrm{CH}_{2}-\right), 1.40-1.21\left(\mathrm{~m}, 6 \mathrm{H},-\left(\mathrm{CH}_{2}\right)_{3}-\right), 1.16\left(\mathrm{t}, J=7.3 \mathrm{~Hz}, 3 \mathrm{H},-\mathrm{OCH}_{2} \mathrm{CH}_{3}\right), 0.95(\mathrm{~d}, J$ $\left.=6.5 \mathrm{~Hz}, 3 \mathrm{H},-\mathrm{CH}\left(\mathrm{CH}_{3}\right) \mathrm{CH}_{2}-\right), 0.87\left(\mathrm{~d}, J=6.6 \mathrm{~Hz}, 6 \mathrm{H},-\mathrm{CH}\left(\mathrm{CH}_{3}\right)_{2}\right) .{ }^{13} \mathrm{C} \mathrm{NMR}\left(125 \mathrm{MHz}, \mathrm{CDCl}_{3}, \delta\right)$ : 166.62 , 147.22, 142.33, 138.31, 124.36, 120.13, 111.23, 103.39, 68.21, 59.72, 52.32, 39.38, 37.41, 36.29, $29.92,28.12,24.78,22.84,22.74,19.82,14.95$.
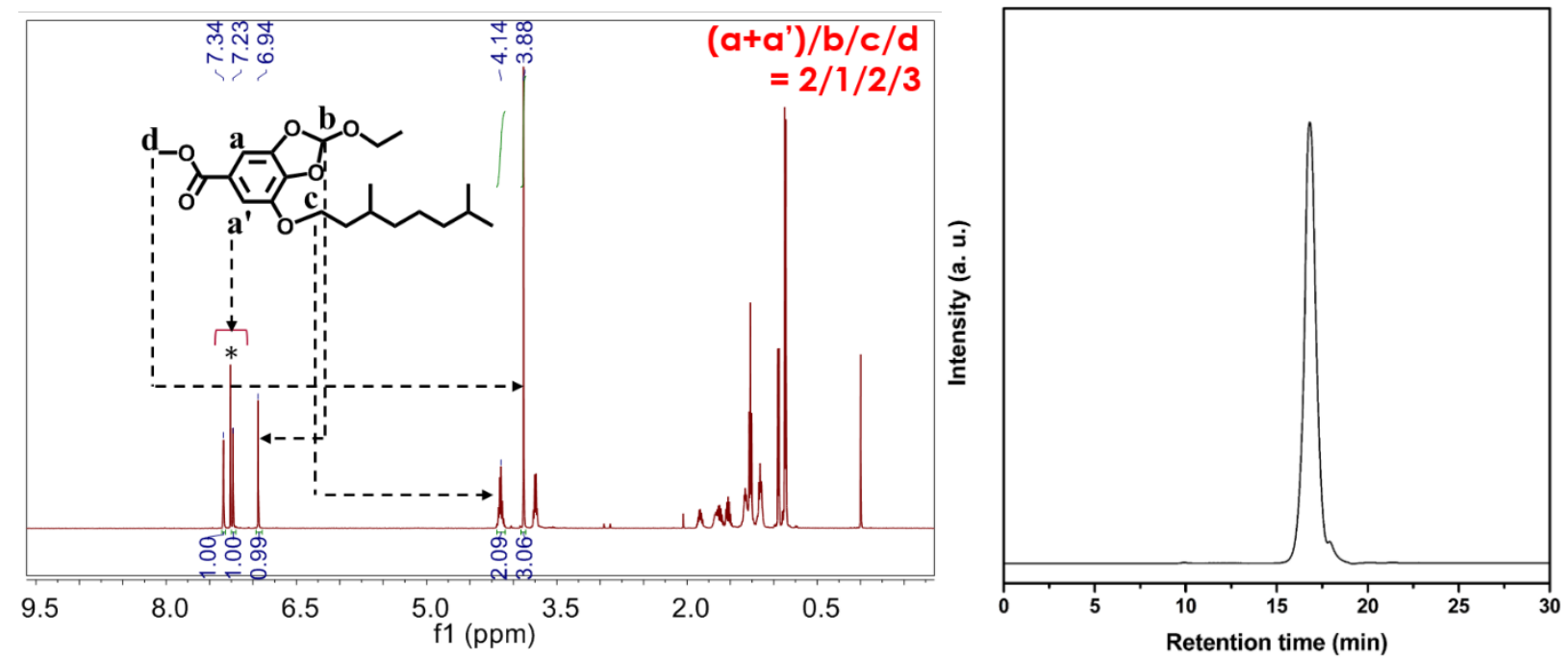

Figure S11. ${ }^{1}$ H NMR spectra (left) and HPLC trace (right) of 7-r. Racemic dimethyloctyl group has been successfully linked to the 5-position of 7-r. 
<smiles>COCCCCCCCCCCOc1cc(C(=O)OC)cc(O)c1O</smiles>

Methyl 3,4-dihydroxy-5-(octyloxy)benzoate (8-8). Compound 8-8 was synthesized by deprotecting the acetal-protected hydroxyl groups of $\mathbf{7 - 8}{ }^{3}$ Briefly, $\mathrm{HCl}$ aqueous solution $(2 \mathrm{M}, 15 \mathrm{~mL})$ was added to a methanol solution $(30 \mathrm{~mL})$ of 7-8 $(1.76 \mathrm{~g}, 5.0 \mathrm{mmol})$. The mixture was stirred at $\mathrm{R}$. T. for $7 \mathrm{~h}$ and then quenched by aqueous $\mathrm{NH}_{3}$ solution until $\mathrm{pH}>7$. Methanol was evaporated in vacuo and the precipitate was washed with a mixed solution of EA and hexanes (1/3). Compound 8-8 was obtained as white powder. Product: 1.26 g. Yield: 85\%. Purity by HPLC: 99+\%. TLC (EA/Hexanes = 1/2): $\mathrm{R}_{\mathrm{f}}=0.45$. M. P.: 121$122{ }^{\circ} \mathrm{C}$.

${ }^{1} \mathrm{H}$ NMR $\left(500 \mathrm{MHz}, \mathrm{CDCl}_{3}, \delta\right): 7.33\left(\mathrm{~s}, 1 \mathrm{H}, \mathrm{ArH}\right.$ para to $\left.-\mathrm{OC}_{8} \mathrm{H}_{17}\right), 7.20\left(\mathrm{~s}, 1 \mathrm{H}, \mathrm{ArH}\right.$ ortho to $\left.-\mathrm{OC}_{8} \mathrm{H}_{17}\right)$, 5.81 (br s, $1 \mathrm{H}, \mathrm{ArOH}), 5.35$ (br s, $1 \mathrm{H}, \mathrm{ArOH}), 4.09$ (t, $\left.J=6.5 \mathrm{~Hz}, 2 \mathrm{H},-\mathrm{OCH}_{2}-\right), 3.88\left(\mathrm{~s}, 3 \mathrm{H},-\mathrm{OCH}_{3}\right)$, 1.85-1.79 (m, 2H, $\left.-\mathrm{OCH}_{2} \mathrm{CH}_{2}-\right), 1.49-1.40\left(\mathrm{~m}, 2 \mathrm{H},-\mathrm{O}\left(\mathrm{CH}_{2}\right)_{2} \mathrm{CH}_{2}-\right), 1.40-1.23\left(\mathrm{~m}, 8 \mathrm{H},-\left(\mathrm{CH}_{2}\right)_{4}-\right), 0.89$ $\left(\mathrm{t}, J=6.9 \mathrm{~Hz}, 3 \mathrm{H},-\mathrm{CH}_{2} \mathrm{CH}_{3}\right) .{ }^{13} \mathrm{C} \mathrm{NMR}\left(125 \mathrm{MHz}, \mathrm{CDCl}_{3}, \delta\right): 166.65,146.12,143.88,137.80,120.87$, 110.48, 105.56, 69.04, 51.53, 31.51, 30.48, 29.05, 28.93, 28.90, 25.64, 22.34, 13.73.
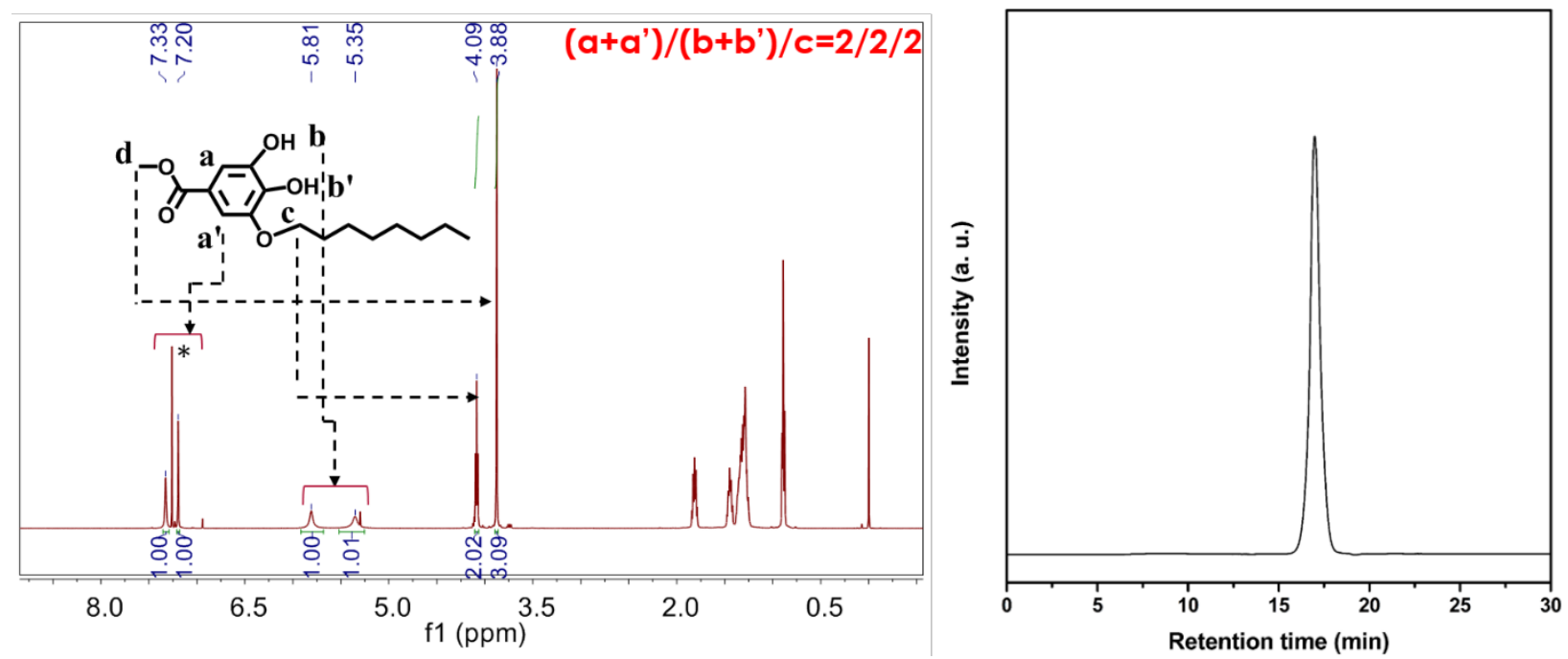

Figure S12. ${ }^{1} \mathrm{H}$ NMR spectra (left) and HPLC trace (right) of 8-8. The hydroxyl groups in the 3- and 4positions have been successfully deprotected in 8-8. 


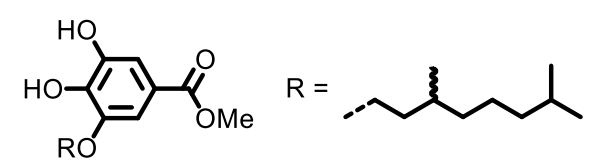

Methyl 3,4-dihydroxy-5-(((rac)-3,7-dimethyloctyl)oxy)benzoate (8-r). Following a procedure similar to that for 8-8, 8-r was obtained as a white solid by deprotecting 7-r (1.90 g, $5.0 \mathrm{mmol})$ in methanol (30 $\mathrm{mL}$ ) using $\mathrm{HCl}$ aqueous solution (2M, $15 \mathrm{~mL}$ ). Product: $1.57 \mathrm{~g}$. Yield: 97\%. Purity by HPLC: 99+\%. TLC $(\mathrm{EA} /$ Hexanes $=1 / 2): \mathrm{R}_{\mathrm{f}}=0.54$. M. P.: $52-53{ }^{\circ} \mathrm{C}$.

${ }^{1} \mathrm{H}$ NMR (500 MHz, $\left.\mathrm{CDCl}_{3}, \delta\right): 7.33$ (d, $J=1.7 \mathrm{~Hz}, 1 \mathrm{H}$, ArH para to -OR), 7.20 (d, J=1.7 Hz, 1H, ArH ortho to -OR), 5.79 (s, $1 \mathrm{H}, \mathrm{ArOH}), 5.35(\mathrm{~s}, 1 \mathrm{H}, \mathrm{ArOH}), 4.17-4.06\left(\mathrm{~m}, 2 \mathrm{H},-\mathrm{OCH}_{2}-\right), 3.88(\mathrm{~s}, 3 \mathrm{H},-$ $\left.\mathrm{OCH}_{3}\right), 1.90-1.80\left(\mathrm{~m}, 1 \mathrm{H},-\mathrm{CH}\left(\mathrm{CH}_{3}\right)_{2}\right), 1.70-1.57\left(\mathrm{~m}, 2 \mathrm{H},-\mathrm{OCH}_{2} \mathrm{CH}_{2}-\right), 1.57-1.48(\mathrm{~m}, 1 \mathrm{H},-$ $\left.\mathrm{CH}\left(\mathrm{CH}_{3}\right) \mathrm{CH}_{2}-\right), 1.39-1.10\left(\mathrm{~m}, 6 \mathrm{H},-\left(\mathrm{CH}_{2}\right)_{3}-\right), 0.95\left(\mathrm{~d}, J=6.3 \mathrm{~Hz}, 3 \mathrm{H},-\mathrm{CH}\left(\mathrm{CH}_{3}\right) \mathrm{CH}_{2}-\right), 0.87(\mathrm{~d}, J=6.6$ $\left.\mathrm{Hz}, 6 \mathrm{H},-\mathrm{CH}\left(\mathrm{CH}_{3}\right)_{2}\right) .{ }^{13} \mathrm{C} \mathrm{NMR}\left(125 \mathrm{MHz}, \mathrm{CDCl}_{3}, \delta\right): 166.87,145.74,143.28,136.85,121.65,110.71$, $105.51,67.72,51.98,39.10,37.12,36.01,29.79,27.87,24.56,22.58,22.48,19.55$.
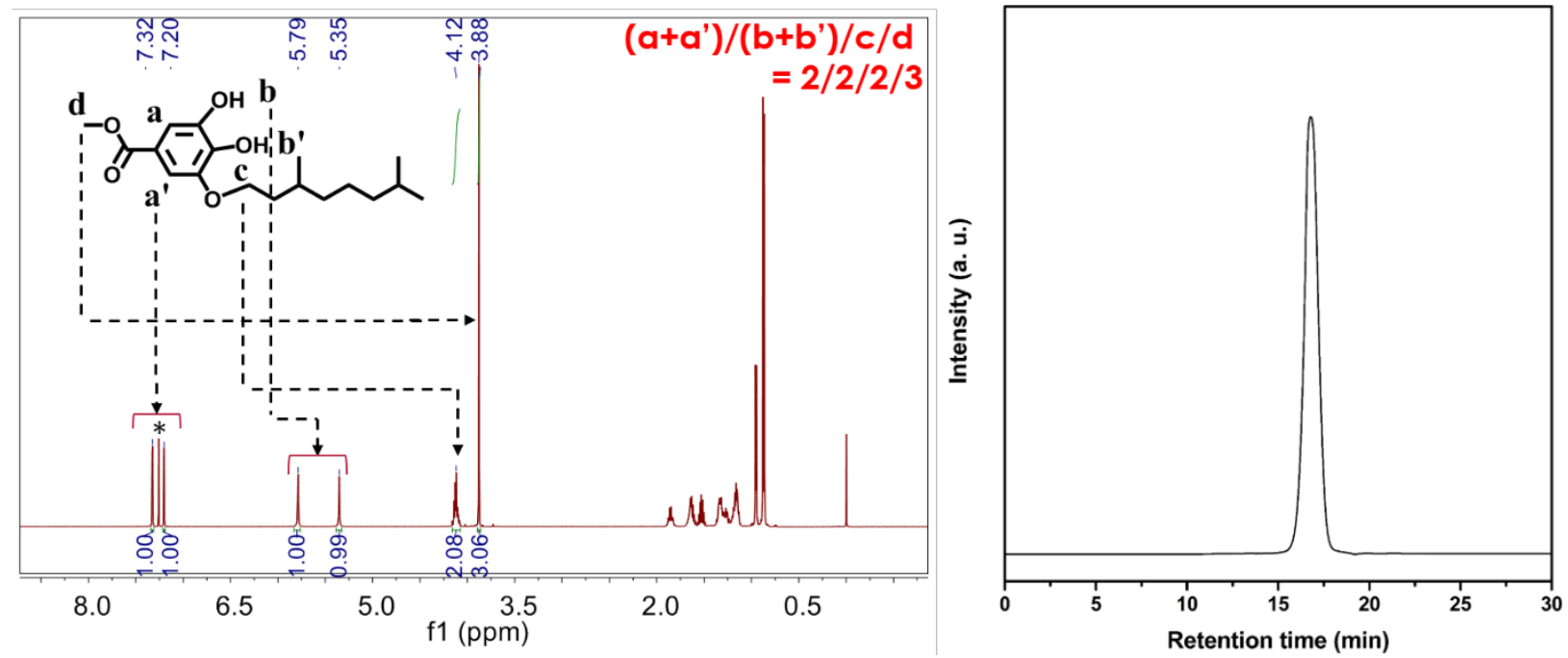

Figure S13. ${ }^{1}$ H NMR spectra (left) and HPLC trace (right) of 8-r. Racemic dimethyloctyl group has been successfully linked to the 5-position of 8-r.

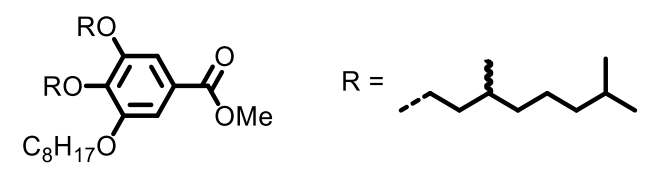

Methyl 3,4-bis(((rac)-3,7-dimethyloctyl)oxy)-5-(octyloxy)benzoate (5-rr8). Compound 5-rr8 was synthesized according to a procedure reported by our group. ${ }^{6}$ A mixture of 8-8 (1.19 g, $\left.4.0 \mathrm{mmol}\right)$ and $\mathrm{K}_{2} \mathrm{CO}_{3}(2.21 \mathrm{~g}, 16.0 \mathrm{mmol})$ was stirred in dry DMF $(20 \mathrm{~mL})$ under $\mathrm{N}_{2}$ protection. (Rac)-1-bromo-3,7dimethyloctane $(1.8 \mathrm{~mL}, 8.4 \mathrm{mmol})$ was added and the reaction mixture was heated at $70{ }^{\circ} \mathrm{C}$ for $4 \mathrm{~h}$. Then the mixture was cooled, filtered, poured into cold water $(100 \mathrm{~mL})$ and extracted with EA $(25 \mathrm{~mL} \times 4)$. The combined EA phase was washed with water $(25 \mathrm{~mL} \times 3)$, dried over anhydrous $\mathrm{MgSO}_{4}$, filtered and evaporated in vacuo. Column chromatography $(\mathrm{EA} / \mathrm{Hexane}=1 / 19)$ gave 5-rr8 as a colorless oil. Product: 2.06 g. Yield: 89\%. Purity by HPLC: 99+\%. TLC (EA/Hexanes =1/9): $\mathrm{R}_{\mathrm{f}}=0.61$. 
${ }^{1} \mathrm{H}$ NMR (500 MHz, $\left.\mathrm{CDCl}_{3}, \delta\right): 7.28-7.23(\mathrm{~m}, 2 \mathrm{H}, \mathrm{ArH}), 4.11-3.98\left(\mathrm{~m}, 6 \mathrm{H},-\mathrm{OCH}_{2}-\right), 3.89(\mathrm{~s}, 3 \mathrm{H},-$ $\left.\mathrm{CO}_{2} \mathrm{CH}_{3}\right), 1.92-1.77\left(\mathrm{~m}, 4 \mathrm{H},-\mathrm{CH}\left(\mathrm{CH}_{3}\right)_{2}\right.$ and $\left.-\mathrm{OCH}_{2} \mathrm{CH}_{2} \mathrm{CH}_{2}-\right), 1.75-1.66\left(\mathrm{~m}, 2 \mathrm{H},-\mathrm{CH}\left(\mathrm{CH}_{3}\right) \mathrm{CH}_{2}-\right)$, 1.66-1.42 (m, 6H, $-\mathrm{OCH}_{2} \mathrm{CH}_{2} \mathrm{CH}\left(\mathrm{CH}_{3}\right)-$ and $\left.-\mathrm{O}\left(\mathrm{CH}_{2}\right)_{2} \mathrm{CH}_{2}-\right), 1.40-1.08\left(\mathrm{~m}, 20 \mathrm{H},-\mathrm{CH}\left(\mathrm{CH}_{3}\right)\left(\mathrm{CH}_{2}\right)_{3}-\right.$ and $\left.-\left(\mathrm{CH}_{2}\right)_{3}\left(\mathrm{CH}_{2}\right)_{4}-\right), 0.95\left(\mathrm{~d}, J=6.2 \mathrm{~Hz}, 3 \mathrm{H},-\mathrm{CH}_{2} \mathrm{CH}\left(\mathrm{CH}_{3}\right) \mathrm{CH}_{2}-\right), 0.92(\mathrm{~d}, J=6.6 \mathrm{~Hz}, 3 \mathrm{H},-$ $\left.\mathrm{CH}_{2} \mathrm{CH}\left(\mathrm{CH}_{3}\right) \mathrm{CH}_{2}-\right), 0.90-0.84\left(\mathrm{~m}, 15 \mathrm{H},-\mathrm{CH}\left(\mathrm{CH}_{3}\right)_{2}-\right.$ and $\left.-\mathrm{CH}_{2} \mathrm{CH}_{3}\right) .{ }^{13} \mathrm{C} \mathrm{NMR}\left(125 \mathrm{MHz}, \mathrm{CDCl}_{3}, \delta\right)$ : 167.13, 153.04, 142.56, 124.87, 108.17, 108.13, 71.89, 69.35, 67.65, 52.28, 39.56, 39.47, 37.68, 37.54, $36.51,32.03,30.03,29.84,29.55,29.51,29.49,28.19,26.29,24.92,22.90,22.87,22.80,19.82,19.79$, 19.75, 14.29. MALDI-TOF MS $m / z$ of $[\mathrm{M}+\mathrm{H}]^{+}$calculated for $\mathrm{C}_{36} \mathrm{H}_{64} \mathrm{O}_{5}$ : 577.5 ; Found: 578.1 .
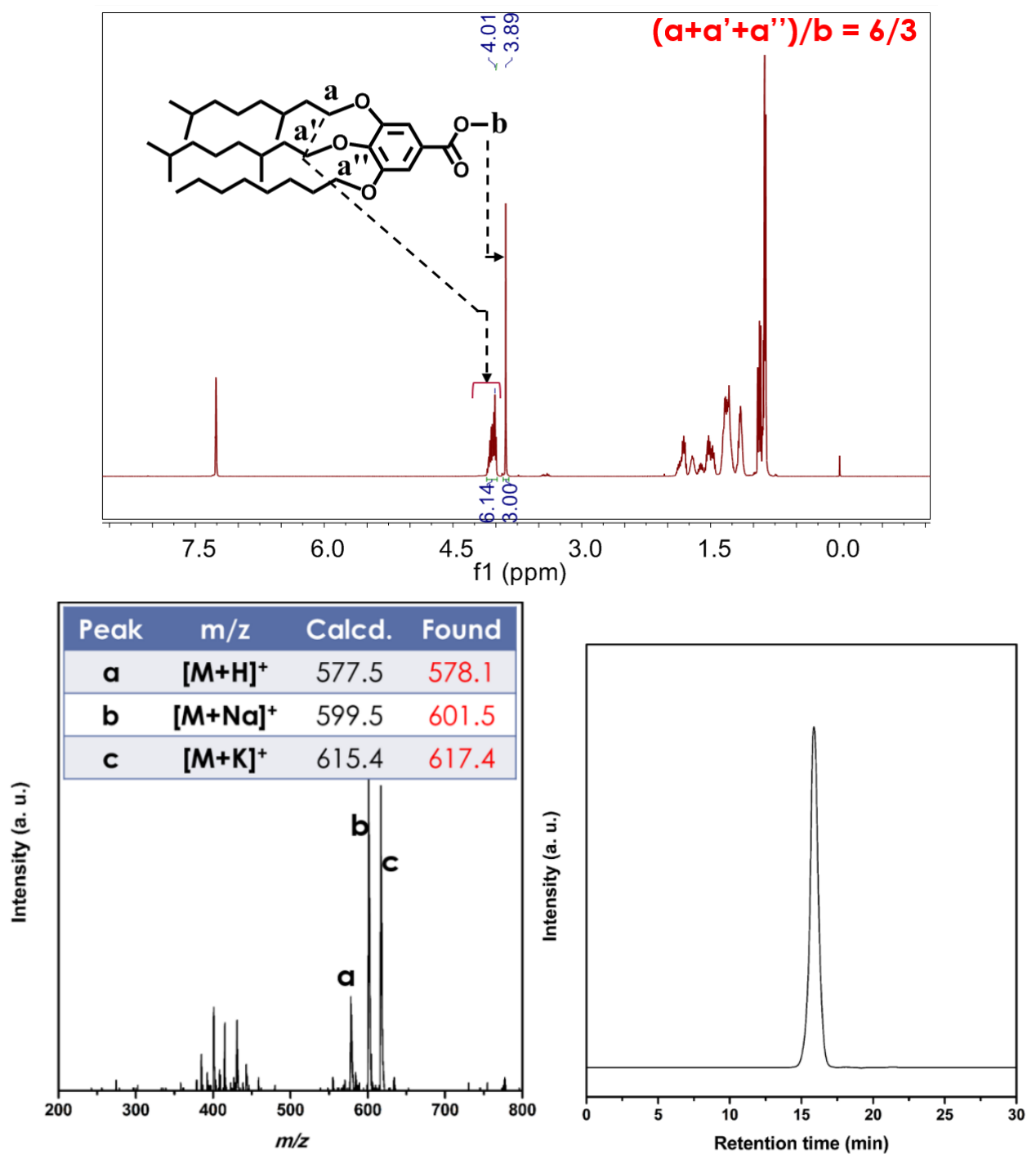

Figure S14. ${ }^{1}$ H NMR spectra (top), MALDI-TOF MS spectra (bottom left) and HPLC trace (bottom right) of 5-rr8. Racemic dimethyloctyl groups have been successfully linked to the 3- and 4-positions in 5-rr8. 


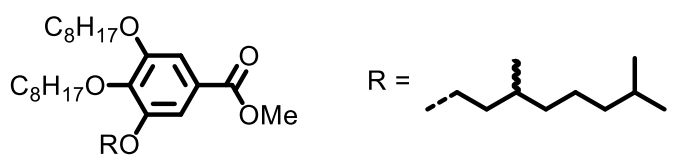

Methyl 3-(((rac)-3,7-dimethyloctyl)oxy)-4,5-bis(octyloxy)benzoate (5-88r). The synthesis procedure for 5-88r is similar to that for 5-rr8. Starting from 8-r $(1.30 \mathrm{~g}, 4.0 \mathrm{mmol})$ and 1-bromooctane $(1.5 \mathrm{~mL}$, $8.4 \mathrm{mmol})$ in the presence of $\mathrm{K}_{2} \mathrm{CO}_{3}(2.21 \mathrm{~g}, 16.0 \mathrm{mmol})$ in dry DMF $(20 \mathrm{~mL}), \mathbf{5 - 8 8} \mathbf{r}$ was obtained as a colorless oil.

${ }^{1} \mathrm{H}$ NMR (500 MHz, $\left.\mathrm{CDCl}_{3}, \delta\right): 7.28-7.23(\mathrm{~m}, 2 \mathrm{H}, \mathrm{Ar} H), 4.10-3.97$ (m, $\left.2 \mathrm{H},-\mathrm{OCH}_{2}-\right), 3.89(\mathrm{~s}, 3 \mathrm{H},-$ $\left.\mathrm{CO}_{2} \mathrm{CH}_{3}\right), 1.91-1.66\left(\mathrm{~m}, 6 \mathrm{H},-\mathrm{OCH}_{2} \mathrm{CH}_{2}-\right), 1.66-1.57\left(\mathrm{~m}, 1 \mathrm{H},-\mathrm{CH}\left(\mathrm{CH}_{3}\right)_{2}\right), 1.57-1.42(\mathrm{~m}, 5 \mathrm{H},-$ $\mathrm{CH}\left(\mathrm{CH}_{3}\right) \mathrm{CH}_{2}-$ and $\left.\mathrm{O}\left(\mathrm{CH}_{2}\right)_{2} \mathrm{CH}_{2}-\right), 1.41-1.19\left(\mathrm{~m}, 22 \mathrm{H},-\mathrm{CH}\left(\mathrm{CH}_{3}\right)\left(\mathrm{CH}_{2}\right)_{3}-\right.$ and $\left.-\left(\mathrm{CH}_{2}\right)_{3}\left(\mathrm{CH}_{2}\right)_{4}-\right), 0.94(\mathrm{~d}$, $\left.J=6.6 \mathrm{~Hz}, 3 \mathrm{H},-\mathrm{CH}_{2} \mathrm{CH}\left(\mathrm{CH}_{3}\right) \mathrm{CH}_{2}-\right), 0.92-0.81\left(\mathrm{~m}, 12 \mathrm{H},-\mathrm{CH}\left(\mathrm{CH}_{3}\right)_{2}\right.$ and $\left.-\mathrm{CH}_{2} \mathrm{CH}_{3}\right) .{ }^{13} \mathrm{C}$ NMR $(125$ $\left.\mathrm{MHz}, \mathrm{CDCl}_{3}, \delta\right): 167.14,153.03,142.57,124.86,108.20,108.15,73.66,69.36,67.67,52.28,39.46,37.52$, $36.52,32.10,32.03,30.54,30.03,29.72,29.57,29.55,29.51,29.49,28.19,26.28,26.26,24.94,22.90$, 22.87, 22.79, 19.81, 14.29. MALDI-TOF MS $m / z$ of $[\mathrm{M}+\mathrm{H}]^{+}$calculated for $\mathrm{C}_{34} \mathrm{H}_{60} \mathrm{O}_{5}$ : 549.5; Found: 550.4 .
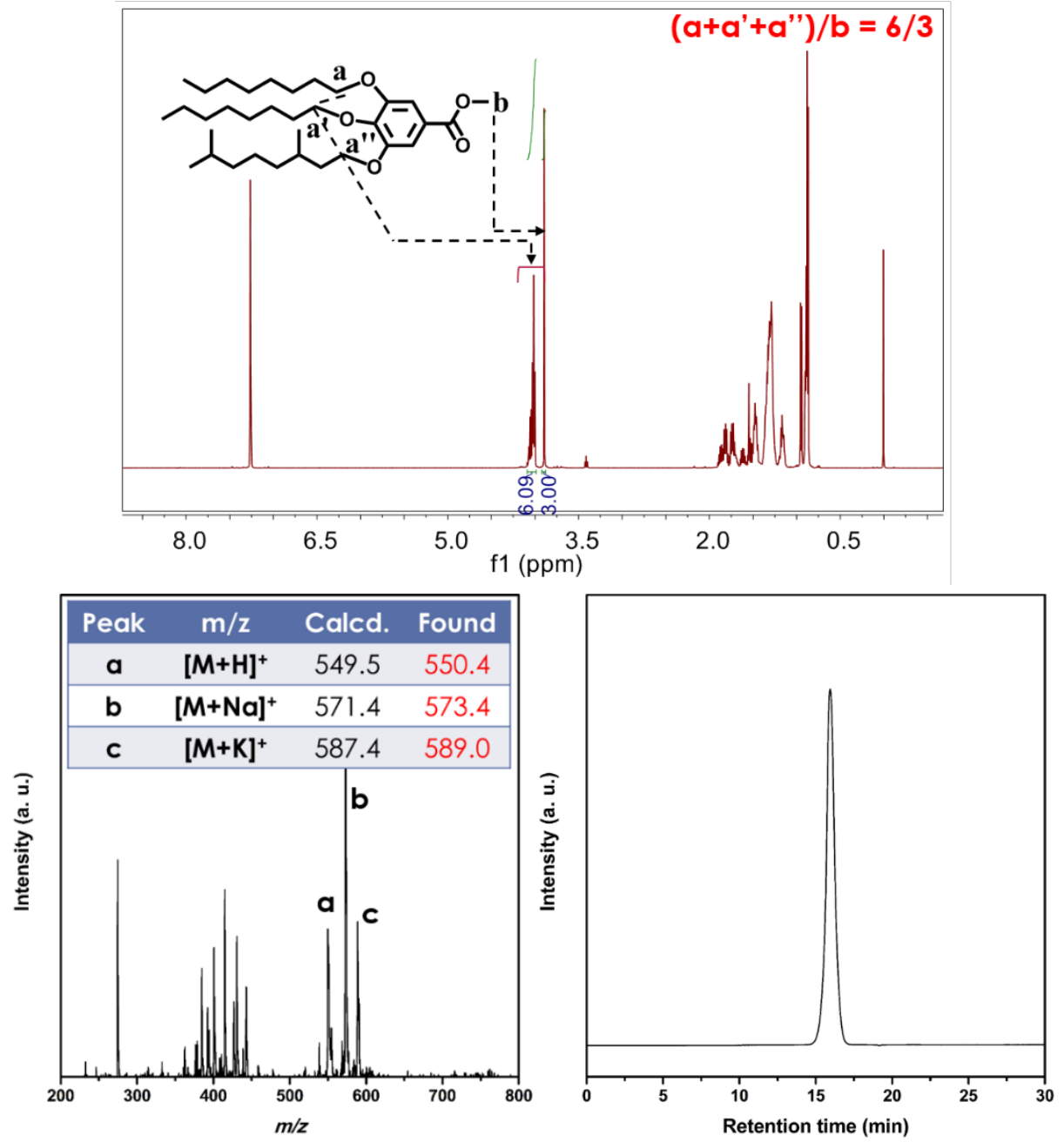

Figure S15. ${ }^{1} \mathrm{H}$ NMR spectra (top), MALDI-TOF MS spectra (bottom left) and HPLC trace (bottom right) of 5-88r. Octyl groups have been successfully linked to the 3- and 4-positions of 5-88r. 
General procedure for preparation of minidendron alcohols 9. The synthesis of minidendron alcohols 9 was conducted according to a procedure reported by our group. ${ }^{7}$ A general example is provided below.

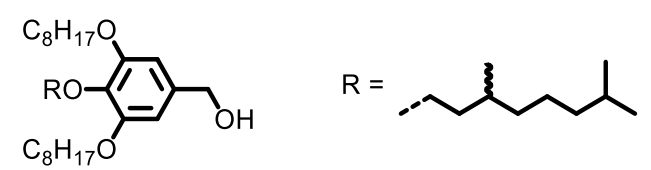

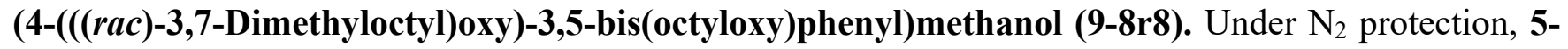
8 r8 $(1.37 \mathrm{~g}, 2.5 \mathrm{mmol})$ in dry THF $(4 \mathrm{~mL})$ was added dropwise to a slurry of $\mathrm{LiAlH}_{4}(0.19 \mathrm{~g}, 5.0 \mathrm{mmol})$ in dry THF $(6 \mathrm{~mL})$ at $0{ }^{\circ} \mathrm{C}$. The mixture was stirred at $\mathrm{R}$. T. for $1 \mathrm{~h}$. Afterwards, the reaction mixture was coolded to $0{ }^{\circ} \mathrm{C}$ and quenched by the successive addition of $\mathrm{H}_{2} \mathrm{O}(0.3 \mathrm{~mL}), 15 \% \mathrm{NaOH}$ aqueous solution $(0.3 \mathrm{~mL})$ and $\mathrm{H}_{2} \mathrm{O}(1.5 \mathrm{~mL})$. Then, the mixture was filtered through celite, washed with plenty of THF, dried over anhydrous $\mathrm{MgSO}_{4}$, filtered and evaporated in vacuo. Compound 9-8r8 was obtained as a white solid. Product: 1.13 g. Yield: 87\%. Purity by HPLC: 99\%. TLC (EA/Hexanes =1/9): $\mathrm{R}_{\mathrm{f}}=0.26$. This was used in the next step without further purification.

${ }^{1} \mathrm{H} \mathrm{NMR}\left(500 \mathrm{MHz}, \mathrm{CDCl}_{3}, \delta\right): 6.56(\mathrm{~s}, 2 \mathrm{H}, \mathrm{ArH}), 4.59$ (d, J=5.6 Hz, 2H, $\left.-\mathrm{CH}_{2} \mathrm{OH}\right), 4.03-3.91(\mathrm{~m}, 6 \mathrm{H}$, $\left.-\mathrm{OCH}_{2} \mathrm{CH}_{2}-\right), 1.88-1.76\left(\mathrm{~m}, 5 \mathrm{H},-\mathrm{CH}\left(\mathrm{CH}_{3}\right)_{2}\right.$ and $\left.-\mathrm{OCH}_{2} \mathrm{CH}_{2} \mathrm{CH}_{2}-\right), 1.75-1.66\left(\mathrm{~m}, 1 \mathrm{H},-\mathrm{CH}\left(\mathrm{CH}_{3}\right) \mathrm{CH}_{2}-\right.$ ), 1.58-1.44 (m, 6H, $-\mathrm{O}\left(\mathrm{CH}_{2}\right)_{2} \mathrm{CH}_{2}-$ and $\left.-\mathrm{OCH}_{2} \mathrm{CH}_{2} \mathrm{CH}\left(\mathrm{CH}_{3}\right)-\right), 1.40-1.09\left(\mathrm{~m}, 22 \mathrm{H},-\mathrm{CH}\left(\mathrm{CH}_{3}\right)\left(\mathrm{CH}_{2}\right)_{3}-\right.$ and $\left.-\left(\mathrm{CH}_{2}\right)_{3}\left(\mathrm{CH}_{2}\right)_{4}-\right), 0.92\left(\mathrm{~d}, J=6.6 \mathrm{~Hz}, 3 \mathrm{H},-\mathrm{CH}_{2} \mathrm{CH}\left(\mathrm{CH}_{3}\right) \mathrm{CH}_{2}-\right), 0.91-0.84\left(\mathrm{~m}, 12 \mathrm{H},-\mathrm{CH}\left(\mathrm{CH}_{3}\right)_{2}\right.$ and $\left.-\mathrm{CH}_{2} \mathrm{CH}_{3}\right) .{ }^{13} \mathrm{C}$ NMR $\left(125 \mathrm{MHz}, \mathrm{CDCl}_{3}, \delta\right): 153.43,137.68,136.34,105.44,71.87,69.25,65.77,39.57$, $37.71,37.53,32.03,29.90,29.61,29.57,29.49,28.19,26.30,24.92,22.89,22.86,22.80,19.75,14.28$. MALDI-TOF MS $m / z$ of $[\mathrm{M}+\mathrm{H}]^{+}$calculated for $\mathrm{C}_{33} \mathrm{H}_{60} \mathrm{O}_{4}$ : 521.5; Found: 521.8 .

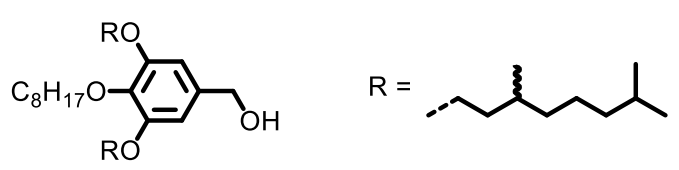

(3,5-bis(((rac)-3,7-Dimethyloctyl)oxy)-4-(octyloxy)phenyl)methanol (9-r8r). From 5-r8r (1.44 g, 2.5 mmol) and $\mathrm{LiAlH}_{4}(0.19 \mathrm{~g}, 5.0 \mathrm{mmol})$ in THF $(10 \mathrm{~mL})$, 9-r8r was obtained as a colorless oil. Product: 1.25 g. Yield: $91 \%$. Purity by HPLC: $99+\%$. TLC $(E A / H e x a n e s=1 / 9): R_{\mathrm{f}}=0.26$.

${ }^{1} \mathrm{H} \mathrm{NMR}\left(500 \mathrm{MHz}, \mathrm{CDCl}_{3}, \delta\right): 6.57$ (s, 2H, $\left.\mathrm{ArH}\right), 4.60$ (d, J=5.9 Hz, 2H, $\left.-\mathrm{CH}_{2} \mathrm{OH}\right), 4.06-3.95(\mathrm{~m}, 4 \mathrm{H}$,

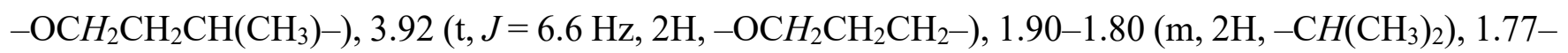
$1.65\left(\mathrm{~m}, 4 \mathrm{H},-\mathrm{CH}\left(\mathrm{CH}_{3}\right) \mathrm{CH}_{2}-\right.$ and $\left.-\mathrm{OCH}_{2} \mathrm{CH}_{2} \mathrm{CH}_{2}-\right), 1.65-1.40\left(\mathrm{~m}, 6 \mathrm{H},-\mathrm{OCH}_{2} \mathrm{CH}_{2} \mathrm{CH}\left(\mathrm{CH}_{3}\right)-\right.$ and $\left.\mathrm{OCH}_{2} \mathrm{CH}_{2} \mathrm{CH}_{2}-\right), 1.40-1.09\left(\mathrm{~m}, 20 \mathrm{H},-\mathrm{CH}\left(\mathrm{CH}_{3}\right)\left(\mathrm{CH}_{2}\right)_{3}-\right.$ and $\left.-\left(\mathrm{CH}_{2}\right)_{3}\left(\mathrm{CH}_{2}\right)_{4}-\right), 0.93(\mathrm{~d}, J=6.7 \mathrm{~Hz}, 6 \mathrm{H},-$ $\left.\mathrm{CH}_{2} \mathrm{CH}\left(\mathrm{CH}_{3}\right) \mathrm{CH}_{2}-\right), 0.91-0.83\left(\mathrm{~m}, 15 \mathrm{H},-\mathrm{CH}\left(\mathrm{CH}_{3}\right)_{2}\right.$ and $\left.-\mathrm{CH}_{2} \mathrm{CH}_{3}\right) .{ }^{13} \mathrm{C} \mathrm{NMR}\left(100 \mathrm{MHz}, \mathrm{CDCl}_{3}, \delta\right)$ : $153.40,137.62,136.33,105.41,73.58,67.54,65.73,39.46,37.51,36.63,32.10,30.50,30.00,29.75$, 29.56, 28.17, 26.31, 24.92, 22.88, 22.77, 19.77, 14.28. MALDI-TOF MS $m / z$ of $[\mathrm{M}+\mathrm{H}]^{+}$calculated for $\mathrm{C}_{35} \mathrm{H}_{64} \mathrm{O}_{4}:$ 549.5; Found: 549.6. 


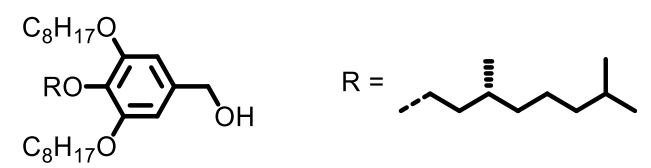

(4-(((S)-3,7-Dimethyloctyl)oxy)-3,5-bis(octyloxy)phenyl)methanol (9-8S8). From 5-8S8 (1.48 g, 2.7 mmol) and $\mathrm{LiAlH}_{4}(0.15 \mathrm{~g}, 4.1 \mathrm{mmol})$ in THF $(10 \mathrm{~mL})$, 9-8S8 was obtained as a white solid. Product: 1.41 g. Yield: $100 \%$. Purity by HPLC: $99+\%$. TLC (EA/Hexanes $=1 / 9): \mathrm{R}_{\mathrm{f}}=0.26$.

${ }^{1} \mathrm{H}$ NMR (400 MHz, $\mathrm{CDCl}_{3}, \delta$ ): 6.56 (s, 2H, $\left.\mathrm{ArH}\right), 4.59$ (s, 2H, $\left.-\mathrm{CH} \mathrm{H}_{2} \mathrm{OH}\right), 4.04-3.90\left(\mathrm{~m}, 6 \mathrm{H},-\mathrm{OCH}_{2}-\right)$, 1.89-1.74 (m, 5H, $-\mathrm{CH}\left(\mathrm{CH}_{3}\right)_{2}$ and $\left.-\mathrm{OCH}_{2} \mathrm{CH}_{2} \mathrm{CH}_{2}-\right), 1.74-1.66\left(\mathrm{~m}, 1 \mathrm{H},-\mathrm{CH}\left(\mathrm{CH}_{3}\right) \mathrm{CH}_{2}-\right), 1.60-1.40(\mathrm{~m}$, $6 \mathrm{H},-\mathrm{OCH}_{2} \mathrm{CH}_{2}-$ and $\left.-\mathrm{OCH}_{2} \mathrm{CH}_{2} \mathrm{CH}_{2}-\right), 1.40-1.07$ (m, 22H, $-\mathrm{CH}\left(\mathrm{CH}_{3}\right)\left(\mathrm{CH}_{2}\right)_{3}-$ and $\left.-\left(\mathrm{CH}_{2}\right)_{3}\left(\mathrm{CH}_{2}\right)_{4}-\right)$, $0.92\left(\mathrm{~d}, J=6.6 \mathrm{~Hz}, 3 \mathrm{H},-\mathrm{CH}_{2} \mathrm{CH}\left(\mathrm{CH}_{3}\right) \mathrm{CH}_{2}-\right), 0.91-0.82\left(\mathrm{~m}, 12 \mathrm{H},-\mathrm{CH}\left(\mathrm{CH}_{3}\right)_{2}\right.$ and $\left.-\mathrm{CH}_{2} \mathrm{CH}_{3}\right) .{ }^{13} \mathrm{C} \mathrm{NMR}$ (100 MHz, $\left.\mathrm{CDCl}_{3}, \delta\right): 155.44,137.74,136.18,105.46,71.82,69.24,65.83,39.52,37.66,37.49,31.98$, 29.86, 29.56, 29.52, 29.44, 28.14, 26.25, 24.87, 22.85, 22.81, 22.75, 19.71, 14.24. MALDI-TOF MS $m / z$ of $[\mathrm{M}+\mathrm{H}]^{+}$calculated for $\mathrm{C}_{33} \mathrm{H}_{60} \mathrm{O}_{4}: 521.5$; Found: 521.6 .

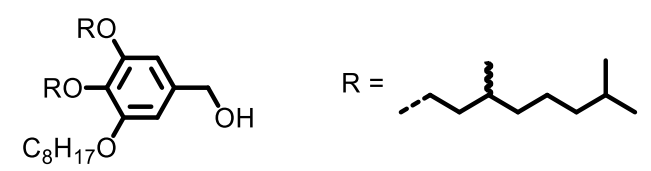

(3,4-Bis(((rac)-3,7-dimethyloctyl)oxy)-5-(octyloxy)phenyl)methanol (9-rr8). From 5-rr8 (1.44 g, 2.5 mmol) and $\mathrm{LiAlH}_{4}(0.19 \mathrm{~g}, 5.0 \mathrm{mmol})$ in THF $(10 \mathrm{~mL})$, 9-rr8 was obtained as a colorless oil. Product: 1.25 g. Yield: 91\%. Purity by HPLC: $99+\%$. TLC $(\mathrm{EA} /$ Hexanes $=1 / 9): \mathrm{R}_{\mathrm{f}}=0.18$.

${ }^{1} \mathrm{H}$ NMR (500 MHz, $\left.\mathrm{CDCl}_{3}, \delta\right): 6.56$ (s, 2H, $\left.\mathrm{ArH}\right), 4.59$ (s, 2H, $\left.-\mathrm{CH}_{2} \mathrm{OH}\right), 4.07-3.91\left(\mathrm{~m}, 6 \mathrm{H},-\mathrm{OCH}_{2}-\right)$, 1.93-1.65 (m, 6H, $-\mathrm{OCH}_{2} \mathrm{CH}_{2} \mathrm{CH}_{2}-,-\mathrm{CH}\left(\mathrm{CH}_{3}\right)_{2}$ and $\left.-\mathrm{CH}\left(\mathrm{CH}_{3}\right) \mathrm{CH}_{2}-\right), 1.65-1.43\left(\mathrm{~m}, 6 \mathrm{H},-\mathrm{O}\left(\mathrm{CH}_{2}\right)_{2} \mathrm{CH}_{2}-\right.$ and $\left.-\mathrm{OCH}_{2} \mathrm{CH}_{2} \mathrm{CH}\left(\mathrm{CH}_{3}\right)-\right), 1.43-1.09\left(\mathrm{~m}, 20 \mathrm{H},-\mathrm{CH}\left(\mathrm{CH}_{3}\right)\left(\mathrm{CH}_{2}\right)_{3}-\right.$ and $\left.-\left(\mathrm{CH}_{2}\right)_{3}\left(\mathrm{CH}_{2}\right)_{4}-\right), 1.00-0.84(\mathrm{~m}$, $\left.21 \mathrm{H},-\mathrm{CH}_{3}\right) .{ }^{13} \mathrm{C} \mathrm{NMR}\left(125 \mathrm{MHz}, \mathrm{CDCl}_{3}, \delta\right): 153.44,137.76,136.20,105.50,71.82,69.26,68.11,67.56$, $65.82,39.53,39.43,37.68,37.50,36.58,31.98,30.47,29.99,29.87,28.14,26.25,25.75,24.87,22.85$, $22.81,22.76,22.74,19.77,19.73,14.23$. MALDI-TOF MS $m / z$ of $[\mathrm{M}+\mathrm{H}]^{+}$calculated for $\mathrm{C}_{35} \mathrm{H}_{64} \mathrm{O}_{4}$ : 549.5; Found: 549.6.

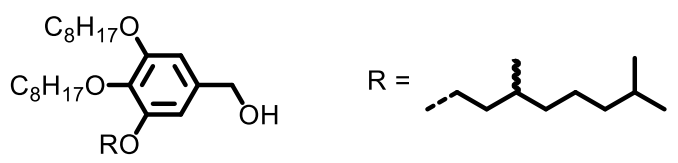

(3-(((rac)-3,7-Dimethyloctyl)oxy)-4,5-bis(octyloxy)phenyl)methanol (9-88r). From 5-88r (1.37 g, 2.5 mmol) and $\mathrm{LiAlH}_{4}(0.19 \mathrm{~g}, 5.0 \mathrm{mmol})$ in THF $(10 \mathrm{~mL}), \mathbf{9 - 8 8 r}$ was obtained as a colorless oil. Product: 1.15 g. Yield: $89 \%$. Purity by HPLC: $99+\%$. TLC $($ EA/Hexanes $=1 / 9): \mathrm{R}_{\mathrm{f}}=0.18$.

${ }^{1} \mathrm{H}$ NMR (500 MHz, $\left.\mathrm{CDCl}_{3}, \delta\right): 6.56(\mathrm{~d}, J=3.7 \mathrm{~Hz}, 2 \mathrm{H}, \mathrm{ArH}), 4.56\left(\mathrm{~s}, 2 \mathrm{H},-\mathrm{CH}_{2} \mathrm{OH}\right), 4.05-3.89(\mathrm{~m}, 6 \mathrm{H}$, $\left.-\mathrm{OCH}_{2}-\right)$, $1.89-1.65\left(\mathrm{~m}, 5 \mathrm{H},-\mathrm{CH}\left(\mathrm{CH}_{3}\right)_{2}\right.$ and $\left.-\mathrm{OCH}_{2} \mathrm{CH}_{2} \mathrm{CH}_{2}-\right), 1.65-1.40\left(\mathrm{~m}, 7 \mathrm{H},-\mathrm{CH}_{\left(\mathrm{CH}_{3}\right) \mathrm{CH}_{2}-,-}\right.$ $\mathrm{O}\left(\mathrm{CH}_{2}\right)_{2} \mathrm{CH}_{2}-$ and $\left.-\mathrm{OCH}_{2} \mathrm{CH}_{2} \mathrm{CH}\left(\mathrm{CH}_{3}\right)-\right), 1.40-1.08\left(\mathrm{~m}, 22 \mathrm{H},-\mathrm{CH}\left(\mathrm{CH}_{3}\right)\left(\mathrm{CH}_{2}\right)_{3}-\right.$ and $\left.-\left(\mathrm{CH}_{2}\right)_{3}\left(\mathrm{CH}_{2}\right)_{4}-\right)$, $0.94\left(\mathrm{~d}, J=6.6 \mathrm{~Hz}, 3 \mathrm{H},-\mathrm{CH}_{2} \mathrm{CH}\left(\mathrm{CH}_{3}\right) \mathrm{CH}_{2}-\right), 0.91-0.82\left(\mathrm{~m}, 12 \mathrm{H},-\mathrm{CH}\left(\mathrm{CH}_{3}\right)_{2}\right.$ and $\left.-\mathrm{CH}_{2} \mathrm{CH}_{3}\right) .{ }^{13} \mathrm{C} \mathrm{NMR}$ (125 MHz, $\left.\mathrm{CDCl}_{3}, \delta\right): 153.45,137.80,136.15,105.56,73.57,69.29,68.13,65.86,39.44,37.49,36.60$, $32.07,31.99,30.49,29.99,29.72,29.58,29.52,29.45,28.15,25.77,24.89,22.85,22.83,22.75,19.77$, 14.26. MALDI-TOF MS $m / z$ of $[\mathrm{M}+\mathrm{H}]^{+}$calculated for $\mathrm{C}_{33} \mathrm{H}_{60} \mathrm{O}_{4}: 521.5$; Found: 521.8 . 
General procedure for preparation of minidendron chlorides $\mathbf{1 0}$. The synthesis of minidendron chlorides 10 was conducted according to a procedure reported by our group. ${ }^{8}$ A general example is provided below.

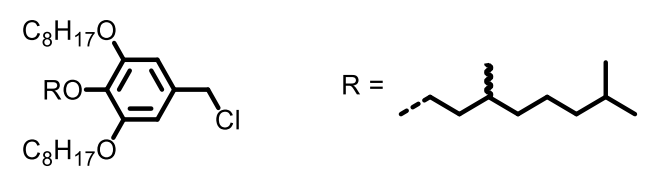

5-(Chloromethyl)-2-(((rac)-3,7-dimethyloctyl)oxy)-1,3-bis(octyloxy)benzene (10-8r8). To a solution of 9-8r8 (1.04 g, $2.0 \mathrm{mmol})$ in dry DCM $(10 \mathrm{~mL})$ was added a catalytic amount of DMF $(30 \mu \mathrm{L})$, followed by the dropwise addition of $\mathrm{SOCl}_{2}(0.22 \mathrm{~mL}, 3.0 \mathrm{mmol})$ with a syringe. Then the reaction mixture was stirred at R. T. for $30 \mathrm{~min}$. After the completion of reaction, the reaction mixture was evaporated in vacuo to remove DCM and excessive $\mathrm{SOCl}_{2}$, giving 10-8r8 as a light yellow oil. Product: $1.08 \mathrm{~g}$. Yield: $100 \%$. Purity by HPLC: $99 \%$. TLC (EA/Hexanes $=1 / 9)$ : $R_{f}=0.79$. This was used in the next step without further purification.

${ }^{1} \mathrm{H}$ NMR (500 MHz, $\left.\mathrm{CDCl}_{3}, \delta\right): 6.57$ (s, 2H, $\left.\mathrm{ArH}\right), 4.51$ (s, 2H, $\left.-\mathrm{CH}_{2} \mathrm{Cl}\right), 4.02-3.92\left(\mathrm{~m}, 6 \mathrm{H},-\mathrm{OCH}_{2}-\right)$, 1.87-1.75 (m, 5H, $-\mathrm{CH}\left(\mathrm{CH}_{3}\right)_{2}$ and $\left.-\mathrm{OCH}_{2} \mathrm{CH}_{2} \mathrm{CH}_{2}-\right), 1.75-1.66\left(\mathrm{~m}, 1 \mathrm{H},-\mathrm{CH}\left(\mathrm{CH}_{3}\right) \mathrm{CH}_{2}-\right), 1.58-1.42(\mathrm{~m}$, $6 \mathrm{H},-\mathrm{O}\left(\mathrm{CH}_{2}\right)_{2} \mathrm{CH}_{2}-$ and $\left.-\mathrm{OCH}_{2} \mathrm{CH}_{2} \mathrm{CH}\left(\mathrm{CH}_{3}\right)_{-}\right), 1.40-1.08\left(\mathrm{~m}, 22 \mathrm{H},-\mathrm{CH}\left(\mathrm{CH}_{3}\right)\left(\mathrm{CH}_{2}\right)_{3}-\right.$ and $\left.\left(\mathrm{CH}_{2}\right)_{3}\left(\mathrm{CH}_{2}\right)_{4}-\right), 0.92\left(\mathrm{~d}, J=6.6 \mathrm{~Hz}, 3 \mathrm{H},-\mathrm{CH}_{2} \mathrm{CH}\left(\mathrm{CH}_{3}\right) \mathrm{CH}_{2}-\right), 0.91-0.82\left(\mathrm{~m}, 12 \mathrm{H},-\mathrm{CH}\left(\mathrm{CH}_{3}\right)_{2}\right.$ and $\left.\mathrm{CH}_{2} \mathrm{CH}_{3}\right) .{ }^{13} \mathrm{C} \mathrm{NMR}\left(125 \mathrm{MHz}, \mathrm{CDCl}_{3}, \delta\right): 153.42,138.51,132.53,107.24,71.86,69.31,47.15,39.56$, $37.70,37.54,32.03,29.89,29.57,29.49,28.19,26.29,24.92,22.90,22.87,22.80,19.75,14.29$. MALDITOF MS $m / z$ of $[\mathrm{M}+\mathrm{H}]^{+}$calculated for $\mathrm{C}_{33} \mathrm{H}_{59} \mathrm{ClO}_{3}$ : 539.4; Found: 540.3 .

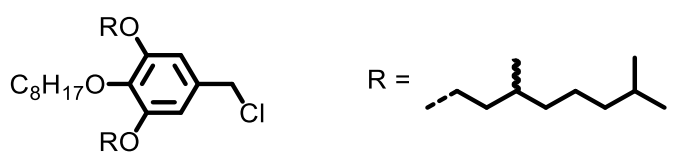

5-(Chloromethyl)-1,3-bis(((rac)-3,7-dimethyloctyl)oxy)-2-(octyloxy)benzene (10-r8r). From 9-r8r $(1.10 \mathrm{~g}, 2.0 \mathrm{mmol})$ and $\mathrm{SOCl}_{2}(0.22 \mathrm{~mL}, 3.0 \mathrm{mmol})$ with a catalytic amount of DMF $(30 \mu \mathrm{L})$ in dry DCM $(10 \mathrm{~mL}), \mathbf{1 0}-\mathbf{r} 8 \mathbf{r}$ was obtained as a light yellow oil. Product: 1.13 g. Yield: 100\%. Purity by HPLC: 99+\%. TLC $(E A / H e x a n e s=1 / 9): R_{f}=0.72$. This was used in the next step without further purification.

${ }^{1} \mathrm{H}$ NMR (500 MHz, $\left.\mathrm{CDCl}_{3}, \delta\right): 6.58(\mathrm{~s}, 2 \mathrm{H}, \mathrm{ArH}), 4.51$ (s, 2H, $\left.-\mathrm{CH}_{2} \mathrm{Cl}\right), 4.07-3.95$ (m, 4H, $\left.\mathrm{OCH}_{2} \mathrm{CH}_{2} \mathrm{CH}\left(\mathrm{CH}_{3}\right)-\right), 3.93\left(\mathrm{t}, J=6.6 \mathrm{~Hz}, 2 \mathrm{H},-\mathrm{OCH}_{2} \mathrm{CH}_{2} \mathrm{CH}_{2}-\right), 1.89-1.80\left(\mathrm{~m}, 2 \mathrm{H},-\mathrm{CH}\left(\mathrm{CH}_{3}\right)_{2}\right), 1.77-$ $1.65\left(\mathrm{~m}, 4 \mathrm{H},-\mathrm{CH}\left(\mathrm{CH}_{3}\right) \mathrm{CH}_{2}-\right.$ and $\left.-\mathrm{OCH}_{2} \mathrm{CH}_{2} \mathrm{CH}_{2}-\right), 1.64-1.42\left(\mathrm{~m}, 6 \mathrm{H},-\mathrm{OCH}_{2} \mathrm{CH}_{2} \mathrm{CH}\left(\mathrm{CH}_{3}\right)-\right.$ and $\left.\mathrm{OCH}_{2} \mathrm{CH}_{2} \mathrm{CH}_{2}-\right), 1.40-1.10\left(\mathrm{~m}, 20 \mathrm{H},-\mathrm{CH}\left(\mathrm{CH}_{3}\right)\left(\mathrm{CH}_{2}\right)_{3}-\right.$ and $\left.-\left(\mathrm{CH}_{2}\right)_{3}\left(\mathrm{CH}_{2}\right)_{4}-\right), 0.94(\mathrm{~d}, J=6.6 \mathrm{~Hz}, 6 \mathrm{H},-$ $\left.\mathrm{CH}_{2} \mathrm{CH}\left(\mathrm{CH}_{3}\right) \mathrm{CH}_{2}-\right), 0.91-0.83\left(\mathrm{~m}, 15 \mathrm{H},-\mathrm{CH}\left(\mathrm{CH}_{3}\right)_{2}\right.$ and $\left.-\mathrm{CH}_{2} \mathrm{CH}_{3}\right) .{ }^{13} \mathrm{C} \mathrm{NMR}\left(125 \mathrm{MHz}, \mathrm{CDCl}_{3}, \delta\right)$ : $153.41,138.51,132.51,107.25,73.60,67.62,47.16,39.47,37.52,36.59,32.11,30.53,30.01,29.75$, 29.57, 28.19, 26.32, 24.94, 22.90, 22.80, 19.81, 14.31. MALDI-TOF MS $m / z$ of $[\mathrm{M}+\mathrm{H}]^{+}$calculated for $\mathrm{C}_{35} \mathrm{H}_{63} \mathrm{ClO}_{3}$ : 567.5; Found: 567.7 . 


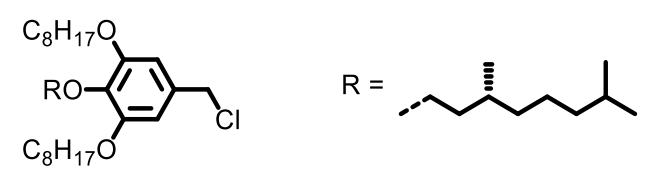

5-(Chloromethyl)-2-(((S)-3,7-dimethyloctyl)oxy)-1,3-bis(octyloxy)benzene (10-8S8). From 9-8S8 $(1.30 \mathrm{~g}, 2.5 \mathrm{mmol})$ and $\mathrm{SOCl}_{2}(0.22 \mathrm{~mL}, 3.0 \mathrm{mmol})$ with a catalytic amount of DMF $(30 \mu \mathrm{L})$ in dry DCM $(10 \mathrm{~mL}), \mathbf{1 0 - 8 S 8}$ was obtained as a light yellow oil. Product: 1.35 g. Yield: 100\%. Purity by HPLC: 99+\%. TLC $($ EA/Hexanes $=1 / 9): \mathrm{R}_{\mathrm{f}}=0.79$. This was used in the next step without further purification.

${ }^{1} \mathrm{H}$ NMR (400 MHz, $\left.\mathrm{CDCl}_{3}, \delta\right): 6.57$ (s, 2H, $\left.\mathrm{ArH}\right), 4.51$ (s, 2H, $\left.-\mathrm{CH}_{2} \mathrm{Cl}\right), 4.05-3.92\left(\mathrm{~m}, 6 \mathrm{H},-\mathrm{OCH}_{2}-\right)$, $1.87-1.76\left(\mathrm{~m}, 5 \mathrm{H},-\mathrm{CH}\left(\mathrm{CH}_{3}\right)_{2}\right.$ and $\left.-\mathrm{OCH}_{2} \mathrm{CH}_{2} \mathrm{CH}_{2}-\right), 1.76-1.66\left(\mathrm{~m}, 1 \mathrm{H},-\mathrm{CH}\left(\mathrm{CH}_{3}\right) \mathrm{CH}_{2}-\right), 1.58-1.41(\mathrm{~m}$, $6 \mathrm{H},-\mathrm{O}\left(\mathrm{CH}_{2}\right)_{2} \mathrm{CH}_{2}-$ and $\left.-\mathrm{OCH}_{2} \mathrm{CH}_{2} \mathrm{CH}\left(\mathrm{CH}_{3}\right)_{-}\right), 1.40-1.08\left(\mathrm{~m}, 22 \mathrm{H},-\mathrm{CH}\left(\mathrm{CH}_{3}\right)\left(\mathrm{CH}_{2}\right)_{3}-\right.$ and $\left.\left(\mathrm{CH}_{2}\right)_{3}\left(\mathrm{CH}_{2}\right)_{4}-\right), 0.92\left(\mathrm{~d}, J=6.6 \mathrm{~Hz}, 3 \mathrm{H},-\mathrm{CH}_{2} \mathrm{CH}\left(\mathrm{CH}_{3}\right) \mathrm{CH}_{2}-\right), 0.91-0.83\left(\mathrm{~m}, 12 \mathrm{H},-\mathrm{CH}\left(\mathrm{CH}_{3}\right)_{2}\right.$ and $\left.\mathrm{CH}_{2} \mathrm{CH}_{3}\right) .{ }^{13} \mathrm{C} \mathrm{NMR}\left(100 \mathrm{MHz}, \mathrm{CDCl}_{3}, \delta\right): 153.37,138.46,132.47,107.20,71.83,69.27,47.13,39.51$, $37.65,37.48,31.98,29.84,29.51,29.43,28.14,26.24,24.87,22.85,22.82,22.75,19.70,14.24$. MALDITOF MS $m / z$ of $[\mathrm{M}+\mathrm{H}]^{+}$calculated for $\mathrm{C}_{33} \mathrm{H}_{59} \mathrm{ClO}_{3}$ : 539.4 ; Found: 539.8 .

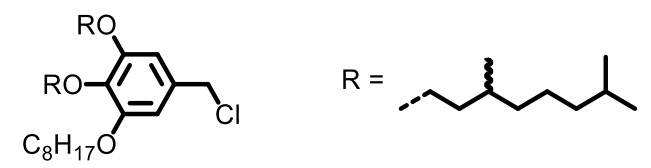

5-(Chloromethyl)-1,2-bis(((rac)-3,7-dimethyloctyl)oxy)-3-(octyloxy)benzene (10-rr8). From 9-rr8 $(1.10 \mathrm{~g}, 2.0 \mathrm{mmol})$ and $\mathrm{SOCl}_{2}(0.22 \mathrm{~mL}, 3.0 \mathrm{mmol})$ with a catalytic amount of DMF $(30 \mu \mathrm{L})$ in dry DCM $(10 \mathrm{~mL}), \mathbf{1 0}-\mathrm{rr8}$ was obtained as a light yellow oil. Product: 1.13 g. Yield: 100\%. Purity by HPLC: 99+\%. TLC $($ EA/Hexanes $=1 / 9): R_{\mathrm{f}}=0.72$. This was used in the next step without further purification.

${ }^{1} \mathrm{H}$ NMR (500 MHz, $\mathrm{CDCl}_{3}, \delta$ ): 6.59 (s, 2H, $\mathrm{ArH}$ ), 4.52 (s, 2H, $-\mathrm{CH}_{2} \mathrm{Cl}$ ), 4.09-3.92 (m, 6H, -OCH $\mathrm{O}_{2}-$ ), 1.93-1.66 (m, 6H, $-\mathrm{OCH}_{2} \mathrm{CH}_{2} \mathrm{CH}_{2}-,-\mathrm{CH}\left(\mathrm{CH}_{3}\right)_{2}$ and $\left.-\mathrm{CH}\left(\mathrm{CH}_{3}\right) \mathrm{CH}_{2}-\right), 1.66-1.43\left(\mathrm{~m}, 6 \mathrm{H},-\mathrm{O}\left(\mathrm{CH}_{2}\right)_{2} \mathrm{CH}_{2}-\right.$ and $\left.-\mathrm{OCH}_{2} \mathrm{CH}_{2} \mathrm{CH}\left(\mathrm{CH}_{3}\right)-\right), 1.43-1.09\left(\mathrm{~m}, 20 \mathrm{H},-\mathrm{CH}\left(\mathrm{CH}_{3}\right)\left(\mathrm{CH}_{2}\right)_{3}-\right.$ and $\left.-\left(\mathrm{CH}_{2}\right)_{3}\left(\mathrm{CH}_{2}\right)_{4}-\right), 1.00-0.82(\mathrm{~m}$, $\left.21 \mathrm{H},-\mathrm{CH}_{3}\right) .{ }^{13} \mathrm{C} \mathrm{NMR}\left(125 \mathrm{MHz}, \mathrm{CDCl}_{3}, \delta\right): 153.38,138.50,132.47,107.24,71.85,69.30,67.61,66.00$, 59.95, 47.14, 39.53, 39.43, 37.67, 37.50, 37.49, 36.54, 31.99, 29.99, 29.86, 28.14, 26.25, 24.87, 22.86, 22.82, 22.76, 22.75, 19.78, 19.73, 15.42, 14.24. MALDI-TOF MS $m / z$ of $[\mathrm{M}+\mathrm{H}]^{+}$calculated for $\mathrm{C}_{35} \mathrm{H}_{63} \mathrm{ClO}_{3}$ : 567.5; Found: 568.0.

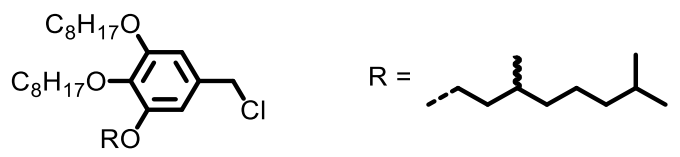

5-(Chloromethyl)-1-(((rac)-3,7-dimethyloctyl)oxy)-2,3-bis(octyloxy)benzene (10-88r). From 9-88r $(1.04 \mathrm{~g}, 2.0 \mathrm{mmol})$ and $\mathrm{SOCl}_{2}(0.22 \mathrm{~mL}, 3.0 \mathrm{mmol})$ with a catalytic amount of DMF $(30 \mu \mathrm{L})$ in dry DCM $(10 \mathrm{~mL}), \mathbf{1 0 - 8 8 r}$ was obtained as a light yellow oil. Product: 1.08 g. Yield: 100\%. Purity by HPLC: 99+\%. TLC $($ EA/Hexanes $=1 / 9): \mathrm{R}_{\mathrm{f}}=0.71$. This was used in the next step without further purification.

${ }^{1} \mathrm{H}$ NMR $\left(500 \mathrm{MHz}, \mathrm{CDCl}_{3}, \delta\right): 6.57(\mathrm{~d}, J=2.1 \mathrm{~Hz}, 2 \mathrm{H}, \mathrm{ArH}), 4.51\left(\mathrm{~s}, 2 \mathrm{H},-\mathrm{CH}_{2} \mathrm{Cl}\right), 4.06-3.90(\mathrm{~m}, 6 \mathrm{H}$, $\left.-\mathrm{OCH}_{2}-\right)$, 1.89-1.66 (m, 5H, $-\mathrm{CH}\left(\mathrm{CH}_{3}\right)_{2}$ and $\left.-\mathrm{OCH}_{2} \mathrm{CH}_{2} \mathrm{CH}_{2}-\right), 1.65-1.41\left(\mathrm{~m}, 7 \mathrm{H},-\mathrm{CH}_{\left(\mathrm{CH}_{3}\right) \mathrm{CH}_{2}-,-}\right.$ $\mathrm{O}\left(\mathrm{CH}_{2}\right)_{2} \mathrm{CH}_{2}-$ and $\left.-\mathrm{OCH}_{2} \mathrm{CH}_{2} \mathrm{CH}\left(\mathrm{CH}_{3}\right)-\right), 1.41-1.10\left(\mathrm{~m}, 22 \mathrm{H},-\mathrm{CH}\left(\mathrm{CH}_{3}\right)\left(\mathrm{CH}_{2}\right)_{3}-\right.$ and $\left.-\left(\mathrm{CH}_{2}\right)_{3}\left(\mathrm{CH}_{2}\right)_{4}-\right)$, $0.94\left(\mathrm{~d}, J=6.6 \mathrm{~Hz}, 3 \mathrm{H},-\mathrm{CH}_{2} \mathrm{CH}\left(\mathrm{CH}_{3}\right) \mathrm{CH}_{2}-\right), 0.93-0.82\left(\mathrm{~m}, 12 \mathrm{H},-\mathrm{CH}\left(\mathrm{CH}_{3}\right)_{2}\right.$ and $\left.-\mathrm{CH}_{2} \mathrm{CH}_{3}\right) .{ }^{13} \mathrm{C} \mathrm{NMR}$ (125 MHz, $\left.\mathrm{CDCl}_{3}, \delta\right): 153.38,138.50,132.46,107.27,73.59,69.31,67.62,66.00,47.14,39.42,37.48$, 
$36.55,32.06,31.98,30.48,29.98,29.70,28.14,26.26,26.24,24.88,22.85,22.82,22.74,19.77$. MALDITOF MS $m / z$ of $[\mathrm{M}+\mathrm{H}]^{+}$calculated for $\mathrm{C}_{33} \mathrm{H}_{59} \mathrm{ClO}_{3}$ : 539.4; Found: 540.4 .

General procedure for preparation of minidendron azides 11. The synthesis of minidendron azides 11 was conducted according to a procedure reported by our group. ${ }^{9}$ A general example is provided below.
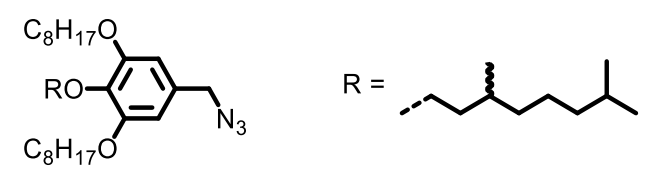

5-(Azidomethyl)-2-(((rac)-3,7-dimethyloctyl)oxy)-1,3-bis(octyloxy)benzene (11-8r8). $\mathrm{NaN}_{3}(0.14 \mathrm{~g}$, $2.2 \mathrm{mmol})$ was added to a solution of $\mathbf{1 0 - 8} 8 \mathbf{8}(0.97 \mathrm{~g}, 1.8 \mathrm{mmol})$ in DMF $(10 \mathrm{~mL})$ and the reaction mixture was stirred at R. T. for $9 \mathrm{~h}$. Then, the reaction mixture was poured into $50 \mathrm{~mL}$ cold water and extracted with EA $(25 \mathrm{~mL} \times 4)$. The combined EA phase was washed with water $(25 \mathrm{~mL} \times 3)$, dried over anhydrous $\mathrm{MgSO}_{4}$, filtered and evaporated in vacuo. Compound 11-8r8 was obtained as a light yellow oil. Product: 0.98 g. Yield: $100 \%$. Purity by HPLC: $99 \%$. TLC $(\mathrm{EA} /$ Hexanes $=1 / 9): \mathrm{R}_{\mathrm{f}}=0.70$. This was used in the next step without further purification.

${ }^{1} \mathrm{H}$ NMR (500 MHz, $\left.\mathrm{CDCl}_{3}, \delta\right): 6.49$ (s, 2H, $\left.\mathrm{ArH}\right), 4.24\left(\mathrm{~s}, 2 \mathrm{H},-\mathrm{CH}_{2} \mathrm{~N}_{3}\right), 4.04-3.93\left(\mathrm{~m}, 6 \mathrm{H},-\mathrm{OCH}_{2}-\right)$, 1.88-1.76 (m, 5H, $-\mathrm{CH}\left(\mathrm{CH}_{3}\right)_{2}$ and $\left.-\mathrm{OCH}_{2} \mathrm{CH}_{2} \mathrm{CH}_{2}-\right), 1.76-1.67\left(\mathrm{~m}, 1 \mathrm{H},-\mathrm{CH}\left(\mathrm{CH}_{3}\right) \mathrm{CH}_{2}-\right), 1.58-1.42(\mathrm{~m}$, $6 \mathrm{H},-\mathrm{O}\left(\mathrm{CH}_{2}\right)_{2} \mathrm{CH}_{2}-$ and $\left.-\mathrm{OCH}_{2} \mathrm{CH}_{2} \mathrm{CH}\left(\mathrm{CH}_{3}\right)_{-}\right), \quad 1.40-1.09\left(\mathrm{~m}, 22 \mathrm{H},-\mathrm{CH}\left(\mathrm{CH}_{3}\right)\left(\mathrm{CH}_{2}\right)_{3}-\right.$ and $\left.\left(\mathrm{CH}_{2}\right)_{3}\left(\mathrm{CH}_{2}\right)_{4}-\right), 0.92\left(\mathrm{~d}, J=6.6 \mathrm{~Hz}, 3 \mathrm{H},-\mathrm{CH}_{2} \mathrm{CH}\left(\mathrm{CH}_{3}\right) \mathrm{CH}_{2}-\right), 0.91-0.84\left(\mathrm{~m}, 12 \mathrm{H},-\mathrm{CH}\left(\mathrm{CH}_{3}\right)_{2}\right.$ and $\left.\mathrm{CH}_{2} \mathrm{CH}_{3}\right) .{ }^{13} \mathrm{C} \mathrm{NMR}\left(125 \mathrm{MHz}, \mathrm{CDCl}_{3}, \delta\right): 153.59,138.38,130.57,106.83,71.87,69.38,55.40,39.58$, 37.72, 37.56, 32.04, 29.92, 29.60, 29.58, 29.50, 28.21, 26.30, 24.94, 22.91, 22.88, 22.82, 19.78, 14.30. MALDI-TOF MS $m / z$ of $[\mathrm{M}+\mathrm{H}]^{+}$calculated for $\mathrm{C}_{33} \mathrm{H}_{59} \mathrm{~N}_{3} \mathrm{O}_{3}$ : 546.5; Found: 546.9.

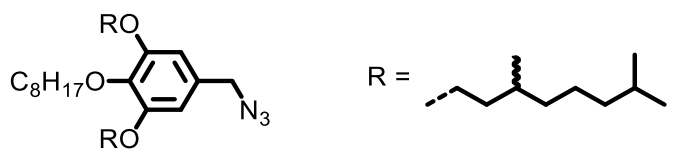

5-(Azidomethyl)-1,3-bis(((rac)-3,7-dimethyloctyl)oxy)-2-(octyloxy)benzene (11-r8r). From 10-r8r $(1.02 \mathrm{~g}, 1.8 \mathrm{mmol})$ and $\mathrm{NaN}_{3}(0.14 \mathrm{~g}, 2.2 \mathrm{mmol})$ in DMF $(10 \mathrm{~mL}), \mathbf{1 1}-\mathbf{r 8 r}$ was obtained as a light yellow oil. Product: 1.02 g. Yield: 99\%. Purity by HPLC: 99\%. TLC (EA/Hexanes $=1 / 9)$ : $\mathrm{R}_{\mathrm{f}}=0.68$. This was used in the next step without further purification.

${ }^{1} \mathrm{H}$ NMR (500 MHz, $\left.\mathrm{CDCl}_{3}, \delta\right): 6.50(\mathrm{~s}, 2 \mathrm{H}, \mathrm{Ar} H), 4.25\left(\mathrm{~s}, 2 \mathrm{H},-\mathrm{CH}_{2} \mathrm{~N}_{3}\right), 4.06-3.96(\mathrm{~m}, 4 \mathrm{H},-$ $\left.\mathrm{OCH}_{2} \mathrm{CH}_{2} \mathrm{CH}\left(\mathrm{CH}_{3}\right)_{-}\right), 3.94\left(\mathrm{t}, J=6.6 \mathrm{~Hz}, 2 \mathrm{H},-\mathrm{OCH}_{2} \mathrm{CH}_{2} \mathrm{CH}_{2}-\right), 1.90-1.81\left(\mathrm{~m}, 2 \mathrm{H},-\mathrm{CH}_{\left.\left(\mathrm{CH}_{3}\right)_{2}\right), 1.77-}\right.$ $1.66\left(\mathrm{~m}, 4 \mathrm{H},-\mathrm{CH}\left(\mathrm{CH}_{3}\right) \mathrm{CH}_{2}-\right.$ and $\left.-\mathrm{OCH}_{2} \mathrm{CH}_{2} \mathrm{CH}_{2}-\right), 1.64-1.41\left(\mathrm{~m}, 6 \mathrm{H},-\mathrm{OCH}_{2} \mathrm{CH}_{2} \mathrm{CH}_{\left(\mathrm{CH}_{3}\right)-\text { and }-}\right.$ $\left.\mathrm{OCH}_{2} \mathrm{CH}_{2} \mathrm{CH}_{2}-\right), 1.38-1.10\left(\mathrm{~m}, 20 \mathrm{H},-\mathrm{CH}\left(\mathrm{CH}_{3}\right)\left(\mathrm{CH}_{2}\right)_{3}-\right.$ and $\left.-\left(\mathrm{CH}_{2}\right)_{3}\left(\mathrm{CH}_{2}\right)_{4}-\right), 0.94(\mathrm{~d}, J=6.6 \mathrm{~Hz}, 6 \mathrm{H},-$ $\left.\mathrm{CH}_{2} \mathrm{CH}\left(\mathrm{CH}_{3}\right) \mathrm{CH}_{2}-\right), 0.91-0.84\left(\mathrm{~m}, 15 \mathrm{H},-\mathrm{CH}\left(\mathrm{CH}_{3}\right)_{2}\right.$ and $\left.-\mathrm{CH}_{2} \mathrm{CH}_{3}\right) .{ }^{13} \mathrm{C} \mathrm{NMR}\left(125 \mathrm{MHz}, \mathrm{CDCl}_{3}, \delta\right)$ : $153.55,138.35,130.56,106.79,73.57,67.65,55.38,39.46,37.52,36.59,32.11,30.53,30.00,29.75$, 29.57, 28.18, 26.31, 24.92, 22.88, 22.78, 19.80, 14.30. MALDI-TOF MS $m / z$ of $[\mathrm{M}+\mathrm{H}]^{+}$calculated for $\mathrm{C}_{35} \mathrm{H}_{63} \mathrm{~N}_{3} \mathrm{O}_{3}$ : 574.5; Found: 575.3. 


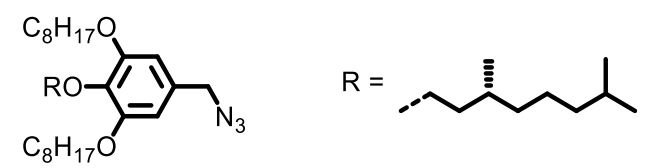

5-(Azidomethyl)-2-(((S)-3,7-dimethyloctyl)oxy)-1,3-bis(octyloxy)benzene (11-8S8). From 10-8S8 (1.24 g, $2.3 \mathrm{mmol})$ and $\mathrm{NaN}_{3}(0.18 \mathrm{~g}, 2.8 \mathrm{mmol})$ in DMF $(10 \mathrm{~mL})$, 11-8S8 was obtained as a light yellow oil. Product: 1.21 g. Yield: 96\%. Purity by HPLC: 99\%. TLC (EA/Hexanes $=1 / 9)$ : $\mathrm{R}_{\mathrm{f}}=0.70$. This was used in the next step without further purification.

${ }^{1} \mathrm{H}$ NMR (500 MHz, $\mathrm{CDCl}_{3}, \delta$ ): 6.53-6.46 (overlapping s, 2H, $\mathrm{ArH}$ ), 4.28-4.21 (overlapping s, 2H, $\left.\mathrm{CH}_{2} \mathrm{~N}_{3}\right), 4.06-3.93\left(\mathrm{~m}, 6 \mathrm{H},-\mathrm{OCH}_{2}-\right), 1.90-1.76\left(\mathrm{~m}, 5 \mathrm{H},-\mathrm{CH}\left(\mathrm{CH}_{3}\right)_{2}\right.$ and $\left.-\mathrm{OCH}_{2} \mathrm{CH}_{2} \mathrm{CH}_{2}-\right), 1.76-1.66$ $\left(\mathrm{m}, 1 \mathrm{H},-\mathrm{CH}\left(\mathrm{CH}_{3}\right) \mathrm{CH}_{2}-\right), 1.62-1.42\left(\mathrm{~m}, 6 \mathrm{H},-\mathrm{O}\left(\mathrm{CH}_{2}\right)_{2} \mathrm{CH}_{2}-\right.$ and $\left.-\mathrm{OCH}_{2} \mathrm{CH}_{2} \mathrm{CH}\left(\mathrm{CH}_{3}\right)-\right), 1.43-1.09(\mathrm{~m}$, $22 \mathrm{H},-\mathrm{CH}\left(\mathrm{CH}_{3}\right)\left(\mathrm{CH}_{2}\right)_{3}-$ and $\left.-\left(\mathrm{CH}_{2}\right)_{3}\left(\mathrm{CH}_{2}\right)_{4}-\right), 0.98-0.83\left(\mathrm{~m}, 15 \mathrm{H},-\mathrm{CH}_{3}\right) .{ }^{13} \mathrm{C} \mathrm{NMR}\left(126 \mathrm{MHz}, \mathrm{CDCl}_{3}\right.$, $\delta): 153.52,138.30,130.50,107.76,71.80,69.31,55.33,39.51,37.65,37.49,31.97,29.85,29.52,29.43$, $29.44,28.14,26.23,24.87,22.84,22.82,22.75,19.70,14.24$. MALDI-TOF MS $\mathrm{m} / z$ of $[\mathrm{M}+\mathrm{H}]^{+}$ calculated for $\mathrm{C}_{33} \mathrm{H}_{59} \mathrm{~N}_{3} \mathrm{O}_{3}$ : 546.5; Found: 546.6.

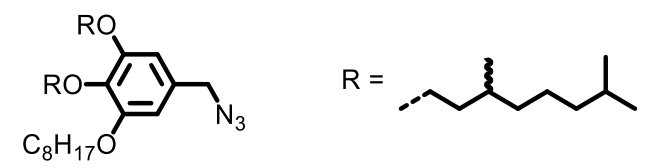

5-(Azidomethyl)-1,2-bis(((rac)-3,7-dimethyloctyl)oxy)-3-(octyloxy)benzene (11-rr8). From 10-rr8 $(1.02 \mathrm{~g}, 1.8 \mathrm{mmol})$ and $\mathrm{NaN}_{3}(0.14 \mathrm{~g}, 2.2 \mathrm{mmol})$ in DMF $(10 \mathrm{~mL}), 11-r r 8$ was obtained as a light yellow oil. Product: 0.98 g. Yield: 95\%. Purity by HPLC: 99\%. TLC (EA/Hexanes $=1 / 9)$ : $R_{f}=0.63$. This was used in the next step without further purification.

${ }^{1} \mathrm{H}$ NMR (500 MHz, $\mathrm{CDCl}_{3}, \delta$ ): 6.52-6.47 (overlapping s, 2H, $\left.\mathrm{ArH}\right), 4.24\left(\mathrm{~s}, 2 \mathrm{H},-\mathrm{CH}_{2} \mathrm{~N}_{3}\right), 4.08-3.92(\mathrm{~m}$, $\left.6 \mathrm{H},-\mathrm{OCH}_{2}-\right)$, 1.93-1.77 (m, 4H, $-\mathrm{OCH}_{2} \mathrm{CH}_{2} \mathrm{CH}_{2}-$ and $\left.-\mathrm{CH}\left(\mathrm{CH}_{3}\right)_{2}\right), 1.75-1.65\left(\mathrm{~m}, 2 \mathrm{H},-\mathrm{CH}_{\left(\mathrm{CH}_{3}\right) \mathrm{CH}_{2}-}\right.$ ), 1.64-1.42 (m, 6H, $-\mathrm{O}\left(\mathrm{CH}_{2}\right)_{2} \mathrm{CH}_{2}-$ and $\left.-\mathrm{OCH}_{2} \mathrm{CH}_{2} \mathrm{CH}\left(\mathrm{CH}_{3}\right)-\right), 1.42-1.08\left(\mathrm{~m}, 20 \mathrm{H},-\mathrm{CH}\left(\mathrm{CH}_{3}\right)\left(\mathrm{CH}_{2}\right)_{3}-\right.$ and $\left.-\left(\mathrm{CH}_{2}\right)_{3}\left(\mathrm{CH}_{2}\right)_{4}-\right), 1.00-0.81\left(\mathrm{~m}, 21 \mathrm{H},-\mathrm{CH}_{3}\right) .{ }^{13} \mathrm{C} \mathrm{NMR}\left(125 \mathrm{MHz}, \mathrm{CDCl}_{3}, \delta\right): 153.53,138.34,130.52$, $106.79,71.82,69.34,67.65,55.36,39.53,39.43,37.67,37.52,37.51,36.55,31.98,28.14,26.25,22.86$, 22.82, 22.75, 19.78, 19.73, 14.24. MALDI-TOF MS $m / z$ of $[\mathrm{M}+\mathrm{H}]^{+}$calculated for $\mathrm{C}_{35} \mathrm{H}_{63} \mathrm{~N}_{3} \mathrm{O}_{3}: 574.5$; Found: 574.7.

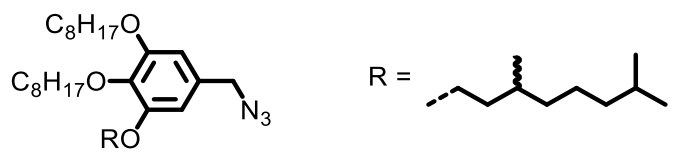

5-(Azidomethyl)-1-(((rac)-3,7-dimethyloctyl)oxy)-2,3-bis(octyloxy)benzene (11-88r). From 10-88r $(0.97 \mathrm{~g}, 1.8 \mathrm{mmol})$ and $\mathrm{NaN}_{3}(0.14 \mathrm{~g}, 2.2 \mathrm{mmol})$ in DMF $(10 \mathrm{~mL}), \mathbf{1 1 - 8 8 r}$ was obtained as a light yellow oil. Product: 0.90 g. Yield: 92\%. Purity by HPLC: 99\%. TLC (EA/Hexanes $=1 / 9)$ : $R_{f}=0.63$. This was used in the next step without further purification.

${ }^{1} \mathrm{H}$ NMR (500 MHz, $\left.\mathrm{CDCl}_{3}, \delta\right): 6.49$ (overlapping s, 2H, $\left.\mathrm{ArH}\right), 4.24\left(\mathrm{~s}, 2 \mathrm{H},-\mathrm{CH}_{2} \mathrm{~N}_{3}\right), 4.07-3.89(\mathrm{~m}, 6 \mathrm{H}$, $\left.-\mathrm{OCH}_{2}-\right)$, 1.91-1.65 (m, 5H, $-\mathrm{CH}\left(\mathrm{CH}_{3}\right)_{2}$ and $\left.-\mathrm{OCH}_{2} \mathrm{CH}_{2} \mathrm{CH}_{2}-\right)$, 1.64-1.42 (m, 7H, $-\mathrm{CH}\left(\mathrm{CH}_{3}\right) \mathrm{CH}_{2}-,-$ $\mathrm{O}\left(\mathrm{CH}_{2}\right)_{2} \mathrm{CH}_{2}-$ and $\left.-\mathrm{OCH}_{2} \mathrm{CH}_{2} \mathrm{CH}\left(\mathrm{CH}_{3}\right)-\right), 1.42-1.10\left(\mathrm{~m}, 22 \mathrm{H},-\mathrm{CH}\left(\mathrm{CH}_{3}\right)\left(\mathrm{CH}_{2}\right)_{3}-\right.$ and $\left.-\left(\mathrm{CH}_{2}\right)_{3}\left(\mathrm{CH}_{2}\right)_{4}-\right)$, $0.95\left(\mathrm{~d}, J=6.6 \mathrm{~Hz}, 3 \mathrm{H},-\mathrm{CH}_{2} \mathrm{CH}\left(\mathrm{CH}_{3}\right) \mathrm{CH}_{2}-\right), 0.93-0.81\left(\mathrm{~m}, 12 \mathrm{H},-\mathrm{CH}\left(\mathrm{CH}_{3}\right)_{2}\right.$ and $\left.-\mathrm{CH}_{2} \mathrm{CH}_{3}\right) .{ }^{13} \mathrm{C} \mathrm{NMR}$ (125 MHz, $\left.\mathrm{CDCl}_{3}, \delta\right): 153.51,130.50,106.82,100.14,73.57,69.35,67.66,55.36,39.42,37.49,36.56$, 
$32.07,31.99,30.49,29.98,28.15,26.27,26.24,24.89,22.85,22.82,22.75,19.77,14.25$. MALDI-TOF $\mathrm{MS} m / z$ of $[\mathrm{M}+\mathrm{H}]^{+}$calculated for $\mathrm{C}_{33} \mathrm{H}_{59} \mathrm{~N}_{3} \mathrm{O}_{3}$ : 546.5; Found: 546.3.

General procedure for preparation of minidendron amines 12. The synthesis of minidendron azides 12 was conducted according to a procedure reported by our group. ${ }^{9}$ A general example is provided below.

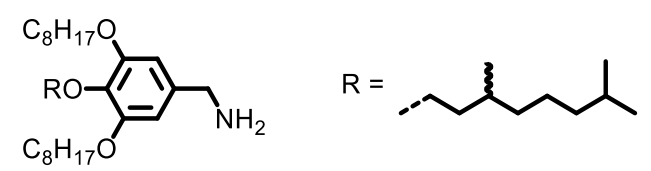

(4-((rac)-3,7-Dimethyloctyl)oxy)-3,5-bis(octyloxy)phenyl)methanamine (12-8r8). Under $\quad \mathrm{N}_{2}$ protection, 11-8r8 $(0.87 \mathrm{~g}, 1.6 \mathrm{mmol})$ in dry THF $(4 \mathrm{~mL})$ was added dropwise to a slurry of $\mathrm{LiAlH}_{4}(0.09$ $\mathrm{g}, 2.4 \mathrm{mmol})$ in dry THF $(6 \mathrm{~mL})$ at $0{ }^{\circ} \mathrm{C}$. The mixture was stirred at $\mathrm{R}$. T. for $1 \mathrm{~h}$. Afterwards, the reaction mixture was cooled to $0{ }^{\circ} \mathrm{C}$ and quenched by the successive addition of $\mathrm{H}_{2} \mathrm{O}(0.15 \mathrm{~mL}), 15 \% \mathrm{NaOH}$ aqueous solution $(0.15 \mathrm{~mL})$ and $\mathrm{H}_{2} \mathrm{O}(0.75 \mathrm{~mL})$. Then, the mixture was filtered through celite, washed with plenty of THF, dried over anhydrous $\mathrm{MgSO}_{4}$, filtered and evaporated in vacuo. Compound 12-8r8 was obtained as a light yellow oil. Product: 0.80 g. Yield: $96 \%$. This was used in the next step without further purification.

${ }^{1} \mathrm{H}$ NMR (500 MHz, $\left.\mathrm{CDCl}_{3}, \delta\right): 6.50(\mathrm{~s}, 2 \mathrm{H}, \mathrm{ArH}), 4.04-3.90\left(\mathrm{~m}, 6 \mathrm{H},-\mathrm{OCH}_{2}-\right), 3.78\left(\mathrm{~s}, 2 \mathrm{H},-\mathrm{CH}_{2} \mathrm{NH}_{2}\right)$, $1.87-1.60\left(\mathrm{~m}, 6 \mathrm{H},-\mathrm{CH}\left(\mathrm{CH}_{3}\right)_{2},-\mathrm{OCH}_{2} \mathrm{CH}_{2} \mathrm{CH}_{2}-\right.$ and $\left.-\mathrm{CH}\left(\mathrm{CH}_{3}\right) \mathrm{CH}_{2}-\right), 1.60-1.40\left(\mathrm{~m}, 6 \mathrm{H},-\mathrm{O}\left(\mathrm{CH}_{2}\right)_{2} \mathrm{CH}_{2}-\right.$ and $\left.-\mathrm{OCH}_{2} \mathrm{CH}_{2} \mathrm{CH}\left(\mathrm{CH}_{3}\right)-\right), 1.40-1.09\left(\mathrm{~m}, 22 \mathrm{H},-\mathrm{CH}\left(\mathrm{CH}_{3}\right)\left(\mathrm{CH}_{2}\right)_{3}-\right.$ and $\left.-\left(\mathrm{CH}_{2}\right)_{3}\left(\mathrm{CH}_{2}\right)_{4}-\right), 0.92(\mathrm{~d}, J=6.6$ $\left.\mathrm{Hz}, 3 \mathrm{H},-\mathrm{CH}_{2} \mathrm{CH}\left(\mathrm{CH}_{3}\right) \mathrm{CH}_{2}-\right), 0.90-0.82\left(\mathrm{~m}, 12 \mathrm{H},-\mathrm{CH}\left(\mathrm{CH}_{3}\right)_{2}\right.$ and $\left.-\mathrm{CH}_{2} \mathrm{CH}_{3}\right) .{ }^{13} \mathrm{C} \mathrm{NMR}(125 \mathrm{MHz}$, $\left.\mathrm{CDCl}_{3}, \delta\right): 153.15,138.43,136.97,105.39,71.58,69.05,46.69,39.29,37.44,37.27,31.75,30.23,29.64$, 27.91, 26.02, 24.63, 22.61, 22.58, 22.51, 19.48, 13.99. MALDI-TOF MS $m / z$ of $[\mathrm{M}+\mathrm{H}]^{+}$calculated for $\mathrm{C}_{33} \mathrm{H}_{61} \mathrm{NO}_{3}$ : 520.5; Found: 520.6.

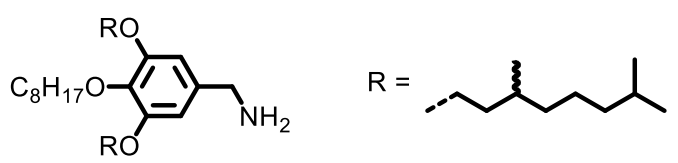

(3,5-bis(((rac)-3,7-Dimethyloctyl)oxy)-4-(octyloxy)phenyl)methanamine (12-r8r). From 11-r8r (0.92 g, $1.6 \mathrm{mmol})$ and $\mathrm{LiAlH}_{4}(0.09 \mathrm{~g}, 2.4 \mathrm{mmol})$ in THF $(10 \mathrm{~mL}), \mathbf{1 2}-\mathbf{r} 8 \mathrm{r}$ was obtained as a colorless oil. Product: 0.87 g. Yield: $96 \%$.

${ }^{1} \mathrm{H}$ NMR (500 MHz, $\left.\mathrm{CDCl}_{3}, \delta\right): 6.51(\mathrm{~s}, 2 \mathrm{H}, \mathrm{ArH}), 4.05-3.90\left(\mathrm{~m}, 6 \mathrm{H},-\mathrm{OCH}_{2}-\right), 3.79\left(\mathrm{~s}, 2 \mathrm{H},-\mathrm{CH}_{2} \mathrm{NH}_{2}\right)$, 1.90-1.80 (m, 2H, $\left.-\mathrm{CH}\left(\mathrm{CH}_{3}\right)_{2}\right), 1.78-1.65\left(\mathrm{~m}, 4 \mathrm{H},-\mathrm{CH}\left(\mathrm{CH}_{3}\right) \mathrm{CH}_{2}-\right.$ and $\left.-\mathrm{OCH}_{2} \mathrm{CH}_{2} \mathrm{CH}_{2}-\right), 1.65-1.41(\mathrm{~m}$, $6 \mathrm{H},-\mathrm{OCH}_{2} \mathrm{CH}_{2} \mathrm{CH}\left(\mathrm{CH}_{3}\right)-$ and $\left.-\mathrm{OCH}_{2} \mathrm{CH}_{2} \mathrm{CH}_{2}-\right), 1.40-1.10\left(\mathrm{~m}, 20 \mathrm{H},-\mathrm{CH}\left(\mathrm{CH}_{3}\right)\left(\mathrm{CH}_{2}\right)_{3}-\right.$ and $\left.\left(\mathrm{CH}_{2}\right)_{3}\left(\mathrm{CH}_{2}\right)_{4}-\right), 0.93\left(\mathrm{~d}, J=6.5 \mathrm{~Hz}, 6 \mathrm{H},-\mathrm{CH}_{2} \mathrm{CH}\left(\mathrm{CH}_{3}\right) \mathrm{CH}_{2}-\right), 0.91-0.80\left(\mathrm{~m}, 15 \mathrm{H},-\mathrm{CH}\left(\mathrm{CH}_{3}\right)_{2}\right.$ and $\left.\mathrm{CH}_{2} \mathrm{CH}_{3}\right) .{ }^{13} \mathrm{C} \mathrm{NMR}\left(125 \mathrm{MHz}, \mathrm{CDCl}_{3}, \delta\right): 153.43,138.58,137.20,105.64,73.59,67.61,46.93,39.47$, $37.53,36.67,32.11,30.53,30.01,29.76,29.57,28.18,28.34,24.93,22.89,22.79,19.81,14.30$. MALDITOF MS $m / z$ of $[\mathrm{M}+\mathrm{H}]^{+}$calculated for $\mathrm{C}_{35} \mathrm{H}_{65} \mathrm{NO}_{3}$ : 548.5; Found: 549.2 . 


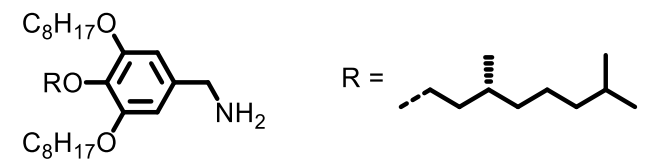

(4-((S)-3,7-Dimethyloctyl)oxy)-3,5-bis(octyloxy)phenyl)methanamine (12-8S8). From 11-8S8 (1.09 $\mathrm{g}, 2.0 \mathrm{mmol})$ and $\mathrm{LiAlH}_{4}(0.11 \mathrm{~g}, 3.0 \mathrm{mmol})$ in THF $(10 \mathrm{~mL}), \mathbf{1 2 - 8 S 8}$ was obtained as a white solid. Product: 1.03 g. Yield: $99 \%$.

${ }^{1} \mathrm{H}$ NMR (500 MHz, $\left.\mathrm{CDCl}_{3}, \delta\right): 6.51(\mathrm{~s}, 2 \mathrm{H}, \mathrm{ArH}), 4.03-3.89\left(\mathrm{~m}, 6 \mathrm{H},-\mathrm{OCH}_{2}-\right), 3.78\left(\mathrm{~s}, 2 \mathrm{H},-\mathrm{CH}_{2} \mathrm{NH}_{2}\right)$, 1.87-1.75 (m, 5H, $-\mathrm{CH}\left(\mathrm{CH}_{3}\right)_{2}$ and $\left.-\mathrm{OCH}_{2} \mathrm{CH}_{2} \mathrm{CH}_{2}-\right), 1.75-1.65\left(\mathrm{~m}, 1 \mathrm{H},-\mathrm{CH}\left(\mathrm{CH}_{3}\right) \mathrm{CH}_{2}-\right), 1.59-1.39(\mathrm{~m}$,

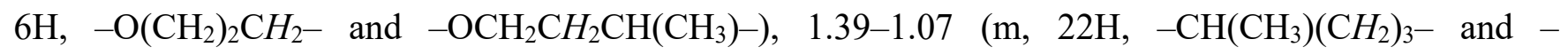
$\left.\left(\mathrm{CH}_{2}\right)_{3}\left(\mathrm{CH}_{2}\right)_{4}-\right), 0.92\left(\mathrm{~d}, J=6.6 \mathrm{~Hz}, 3 \mathrm{H},-\mathrm{CH}_{2} \mathrm{CH}\left(\mathrm{CH}_{3}\right) \mathrm{CH}_{2}-\right), 0.90-0.82\left(\mathrm{~m}, 12 \mathrm{H},-\mathrm{CH}\left(\mathrm{CH}_{3}\right)_{2}\right.$ and $\left.\mathrm{CH}_{2} \mathrm{CH}_{3}\right) .{ }^{13} \mathrm{C}$ NMR $\left(125 \mathrm{MHz}, \mathrm{CDCl}_{3}, \delta\right): 153.37,138.58,137.21,105.64,71.79,69.27,68.08,46.88$, 39.51, 37.67, 37.50, 31.97, 30.46, 29.87, 29.61, 29.52, 29.43, 28.13, 26.25, 25.74, 24.86, 22.83, 22.80, 22.74, 19.71, 14.21. MALDI-TOF MS m/z of $[\mathrm{M}+\mathrm{H}]^{+}$calculated for $\mathrm{C}_{33} \mathrm{H}_{61} \mathrm{NO}_{3}$ : 520.5; Found: 520.6.

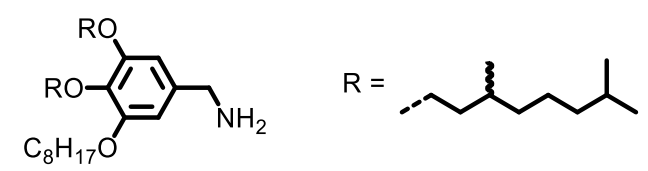

(3,4-Bis(((rac)-3,7-dimethyloctyl)oxy)-5-(octyloxy)phenyl)methanamine (12-rr8). From 11-rr8 (0.92 g, $1.6 \mathrm{mmol})$ and $\mathrm{LiAlH}_{4}(0.09 \mathrm{~g}, 2.4 \mathrm{mmol})$ in THF $(10 \mathrm{~mL})$, 12-rr8 was obtained as a light yellow oil. Product: 0.84 g. Yield: $96 \%$.

${ }^{1} \mathrm{H}$ NMR (500 MHz, $\left.\mathrm{CDCl}_{3}, \delta\right): 6.51(\mathrm{~s}, 2 \mathrm{H}, \mathrm{ArH}), 4.07-3.90\left(\mathrm{~m}, 6 \mathrm{H},-\mathrm{OCH}_{2}-\right), 3.78\left(\mathrm{~s}, 2 \mathrm{H},-\mathrm{CH}_{2} \mathrm{NH}_{2}\right)$, 1.90-1.76 (m, 4H, $-\mathrm{OCH}_{2} \mathrm{CH}_{2} \mathrm{CH}_{2}-$ and $\left.-\mathrm{CH}\left(\mathrm{CH}_{3}\right)_{2}\right), 1.76-1.65\left(\mathrm{~m}, 2 \mathrm{H},-\mathrm{CH}\left(\mathrm{CH}_{3}\right) \mathrm{CH}_{2}-\right), 1.65-1.40(\mathrm{~m}$, $6 \mathrm{H},-\mathrm{O}\left(\mathrm{CH}_{2}\right)_{2} \mathrm{CH}_{2}-$ and $\left.-\mathrm{OCH}_{2} \mathrm{CH}_{2} \mathrm{CH}\left(\mathrm{CH}_{3}\right)-\right), 1.40-1.10\left(\mathrm{~m}, 20 \mathrm{H},-\mathrm{CH}\left(\mathrm{CH}_{3}\right)\left(\mathrm{CH}_{2}\right)_{3}-\right.$ and $\left.\left(\mathrm{CH}_{2}\right)_{3}\left(\mathrm{CH}_{2}\right)_{4}-\right), 0.97-0.80\left(\mathrm{~m}, 21 \mathrm{H},-\mathrm{CH}_{3}\right)$.

${ }^{13} \mathrm{C} \mathrm{NMR}\left(125 \mathrm{MHz}, \mathrm{CDCl}_{3}, \delta\right): 153.40,138.61,137.21,105.64,105.60,105.60,71.82,69.29,68.12$, $67.58,46.91,39.54,39.45,37.69,37.68,37.52,37.50,36.64,32.00,30.48,30.00,29.89,29.62,29.54$, $29.45,28.15,26.27,25.77,25.03,24.89,24.88,22.86,22.82,22.77,22.75,19.79,19.75,14.25$. MALDITOF MS $m / z$ of $[\mathrm{M}+\mathrm{H}]^{+}$calculated for $\mathrm{C}_{35} \mathrm{H}_{65} \mathrm{NO}_{3}$ : 548.5; Found: 548.8 .

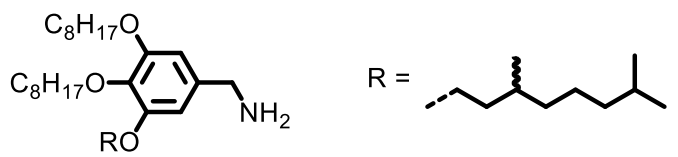

(3-((rac)-3,7-Dimethyloctyl)oxy)-4,5-bis(octyloxy)phenyl)methanamine (12-88r). From 11-88r (0.92 g, $1.6 \mathrm{mmol})$ and $\mathrm{LiAlH}_{4}(0.09 \mathrm{~g}, 2.4 \mathrm{mmol})$ in THF $(10 \mathrm{~mL})$, 12-88r was obtained as a light yellow oil. Product: 0.84 g. Yield: $96 \%$.

${ }^{1} \mathrm{H}$ NMR (500 MHz, $\left.\mathrm{CDCl}_{3}, \delta\right): 6.51(\mathrm{~s}, 2 \mathrm{H}, \mathrm{ArH}), 4.05-3.88\left(\mathrm{~m}, 6 \mathrm{H},-\mathrm{OCH}_{2}-\right), 3.78\left(\mathrm{~s}, 2 \mathrm{H},-\mathrm{CH}_{2} \mathrm{NH}_{2}\right)$, 1.89-1.64 (m, 5H, $-\mathrm{CH}\left(\mathrm{CH}_{3}\right)_{2}$ and $\left.-\mathrm{OCH}_{2} \mathrm{CH}_{2} \mathrm{CH}_{2}-\right), 1.64-1.40\left(\mathrm{~m}, 7 \mathrm{H},-\mathrm{CH}\left(\mathrm{CH}_{3}\right) \mathrm{CH}_{2}-,-\mathrm{O}_{\left(\mathrm{CH}_{2}\right)_{2} \mathrm{CH}_{2}-}\right.$ and $\left.-\mathrm{OCH}_{2} \mathrm{CH}_{2} \mathrm{CH}\left(\mathrm{CH}_{3}\right)-\right), 1.40-1.09\left(\mathrm{~m}, 22 \mathrm{H},-\mathrm{CH}\left(\mathrm{CH}_{3}\right)\left(\mathrm{CH}_{2}\right)_{3}-\right.$ and $\left.-\left(\mathrm{CH}_{2}\right)_{3}\left(\mathrm{CH}_{2}\right)_{4}-\right), 0.94(\mathrm{~d}, J=6.6$ $\left.\mathrm{Hz}, 3 \mathrm{H},-\mathrm{CH}_{2} \mathrm{CH}\left(\mathrm{CH}_{3}\right) \mathrm{CH}_{2}-\right), 0.91-0.81\left(\mathrm{~m}, 12 \mathrm{H},-\mathrm{CH}\left(\mathrm{CH}_{3}\right)_{2}\right.$ and $\left.-\mathrm{CH}_{2} \mathrm{CH}_{3}\right) .{ }^{13} \mathrm{C} \mathrm{NMR}(125 \mathrm{MHz}$, $\left.\mathrm{CDCl}_{3}, \delta\right):$ 13C NMR (125 MHz, CDCl3) $\delta 153.37,138.64,137.19,105.61,73.56,69.30,67.60,46.92$, $39.43,37.49$, 36.64, 32.06, 31.99, 30.49, 30.47, 29.98, 29.72, 29.62, 29.53, 29.45, 28.14, 26.30, 26.26, 
24.88, 22.85, 22.82, 22.74, 19.77, 14.25. MALDI-TOF MS $m / z$ of $[\mathrm{M}+\mathrm{H}]^{+}$calculated for $\mathrm{C}_{33} \mathrm{H}_{61} \mathrm{NO}_{3}$ : 520.5; Found: 520.7.

General procedure for preparation of dendronized PBIs. The synthesis of dendronized PBIs was conducted according to a procedure reported by our group. ${ }^{9}$ A general example is provided below.

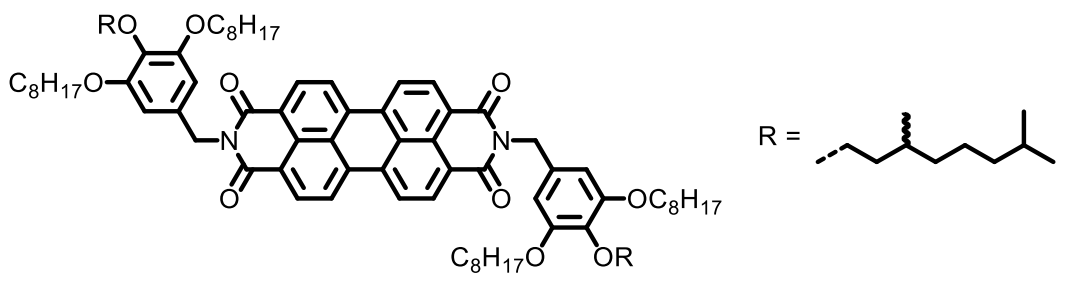

8r8-PBI. A mixture of 12-8r8 (0.62 g, $1.2 \mathrm{mmol})$, PTCDA (0.21 g, $0.54 \mathrm{mmol}), \mathrm{Zn}(\mathrm{OAc})_{2} .2 \mathrm{H}_{2} \mathrm{O}(0.12 \mathrm{~g}$, $0.54 \mathrm{mmol})$ and quinoline $(8 \mathrm{~mL})$ was stirred at $180{ }^{\circ} \mathrm{C}$ for $6 \mathrm{~h}$ under $\mathrm{N}_{2}$ protection. Then the reaction mixture was cooled to $\mathrm{R}$. T. and poured into $1 \mathrm{M} \mathrm{HCl}(45 \mathrm{~mL})$. The precipitate was filtered, washed with water $(30 \mathrm{~mL})$ and methanol $(30 \mathrm{~mL})$ and dried. The crude product was subjected to column chromatography twice using DCM as eluent. The product was purified further by being dissolved in THF and precipitated by the addition of methanol. Target compound 8r8-PBI was obtained as red powder. Product: 0.21 g. Yield: $25 \%$. Purity by HPLC: $99+\%$.

${ }^{1} \mathrm{H}$ NMR (500 MHz, $\mathrm{CDCl}_{3}, \delta$ ): 8.63 (d, $J=8.0 \mathrm{~Hz}, 4 \mathrm{H}, \mathrm{PBI}, 1,6,7,12$-positions), 8.50 (d, $J=8.1 \mathrm{~Hz}, 4 \mathrm{H}$, PBI, 2,5,8,11-positions), 6.84 (s, 4H, $\mathrm{ArH}$ of dendrons), 5.29 (s, 4H, -NCH $\mathrm{N}_{2}$ ), 4.06-3.87 (m, 12H, $\left.\mathrm{OCH}_{2}-\right), 1.85-1.60\left(\mathrm{~m}, 12 \mathrm{H},-\mathrm{CH}\left(\mathrm{CH}_{3}\right)_{2},-\mathrm{OCH}_{2} \mathrm{CH}_{2} \mathrm{CH}_{2}-\right.$ and $\left.-\mathrm{CH}\left(\mathrm{CH}_{3}\right) \mathrm{CH}_{2}-\right), 1.57-1.40(\mathrm{~m}, 12 \mathrm{H},-$ $\mathrm{O}\left(\mathrm{CH}_{2}\right)_{2} \mathrm{CH}_{2}-$ and $\left.-\mathrm{OCH}_{2} \mathrm{CH}_{2} \mathrm{CH}\left(\mathrm{CH}_{3}\right)-\right), 1.39-1.04\left(\mathrm{~m}, 44 \mathrm{H},-\mathrm{CH}\left(\mathrm{CH}_{3}\right)\left(\mathrm{CH}_{2}\right)_{3}-\right.$ and $\left.-\left(\mathrm{CH}_{2}\right)_{3}\left(\mathrm{CH}_{2}\right)_{4}-\right)$, 0.93-0.76 (m, 30H, $\left.-\mathrm{CH}_{3}\right) .{ }^{13} \mathrm{C} \mathrm{NMR}\left(125 \mathrm{MHz}, \mathrm{CDCl}_{3}, \delta\right): 163.13,153.20,134.27,132.28,131.38$, $128.95,125.96,123.16,122.90,108.55,71.84,69.37$, 43.98, 39.49, 37.66, 32.00, 29.89, 29.64, 29.59, $29.47,28.11,26.32,24.84,22.82,22.72,19.70,14.25$. MALDI-TOF MS $m / z$ of $[\mathrm{M}+\mathrm{Na}]^{+}$calculated for $\mathrm{C}_{90} \mathrm{H}_{126} \mathrm{~N}_{2} \mathrm{O}_{10}$ : 1417.9; Found: 1418.5 . 

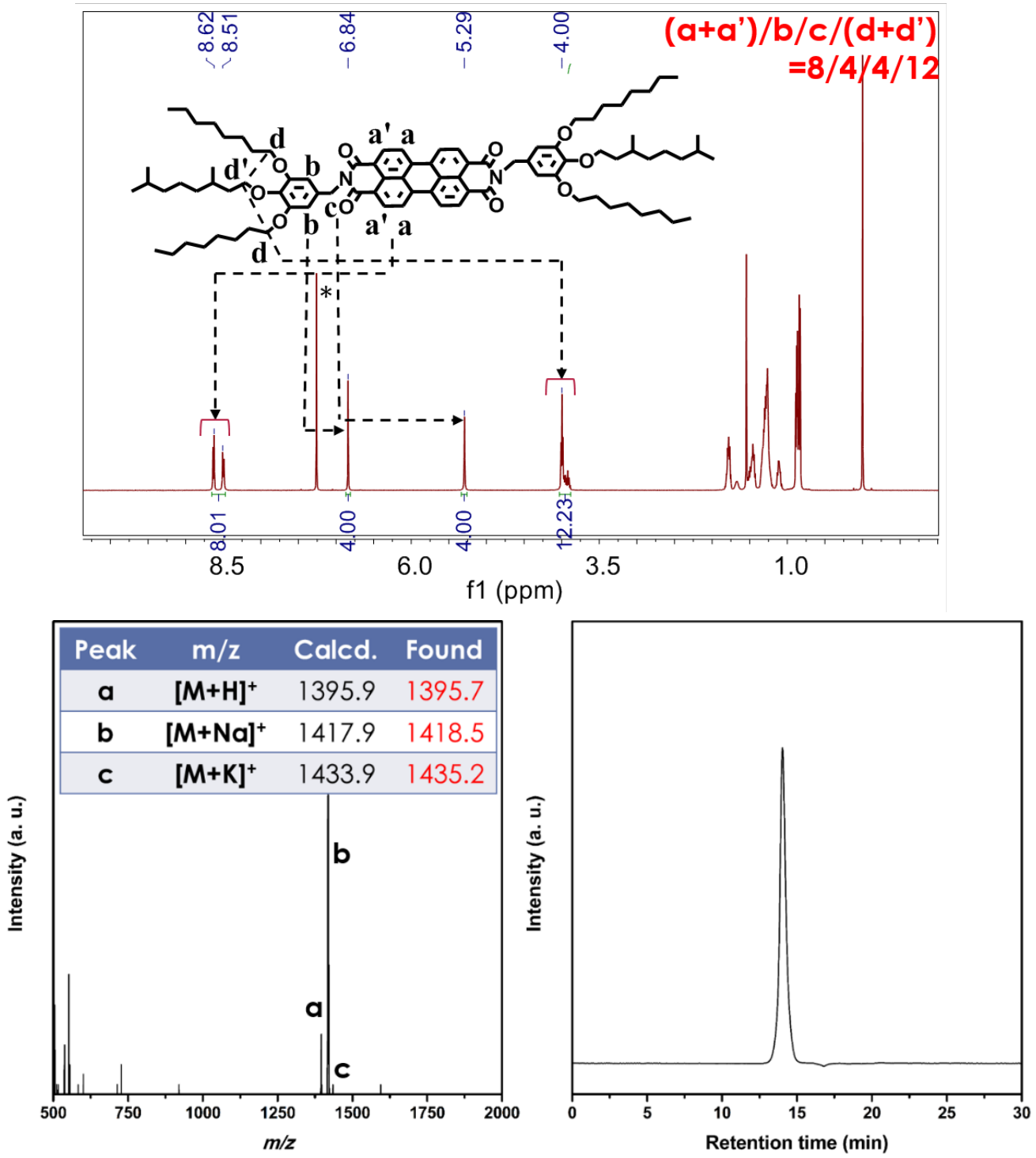

Figure S16. ${ }^{1} \mathrm{H}$ NMR spectra (top), MALDI-TOF MS spectra (bottom left) and HPLC trace (bottom right) of 8r8-PBI. Target molecule 8r8-PBI has been successfully synthesized. 


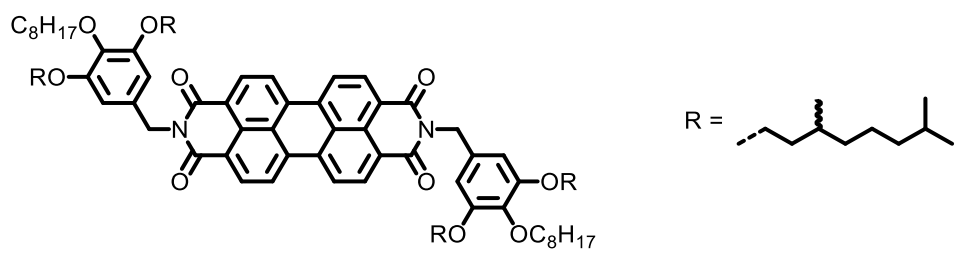

r8r-PBI. From 12-r8r (0.66 g, $1.2 \mathrm{mmol})$, PTCDA (0.21 g, $0.54 \mathrm{mmol})$ and $\mathrm{Zn}(\mathrm{OAc})_{2} .2 \mathrm{H}_{2} \mathrm{O}(0.12 \mathrm{~g}$, $0.54 \mathrm{mmol})$ in quinoline $(8 \mathrm{~mL}), \mathbf{r 8 r}-\mathbf{P B I}$ was obtained as red powder. Product: $0.50 \mathrm{~g}$. Yield: $57 \%$.

Purity by HPLC: $99+\%$.

${ }^{1} \mathrm{H}$ NMR (500 MHz, $\left.\mathrm{CDCl}_{3}, \delta\right): 8.52$ (d, J=7.9 Hz, 4H, PBI, 1,6,7,12-positions), 8.34 (d, J= 7.6 Hz, 4H, PBI, 2,5,8,11-positions), 6.87 (s, 4H, $\mathrm{ArH}$ of dendrons), 5.29 (s, 4H, -NCH $\mathrm{N}_{2}$ ), 4.10-3.98 (m, 8H, -

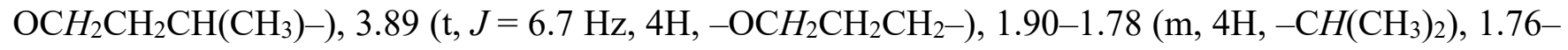
$1.65\left(\mathrm{~m}, 8 \mathrm{H},-\mathrm{CH}\left(\mathrm{CH}_{3}\right) \mathrm{CH}_{2}-\right.$ and $\left.-\mathrm{OCH}_{2} \mathrm{CH}_{2} \mathrm{CH}_{2}-\right), 1.64-1.38\left(\mathrm{~m}, 12 \mathrm{H},-\mathrm{OCH}_{2} \mathrm{CH}_{2} \mathrm{CH}_{\left(\mathrm{CH}_{3}\right)-\text { and }-}\right.$ $\left.\mathrm{OCH}_{2} \mathrm{CH}_{2} \mathrm{CH}_{2}-\right)$, 1.37-1.07 (m, 40H, $-\mathrm{CH}\left(\mathrm{CH}_{3}\right)\left(\mathrm{CH}_{2}\right)_{3}-$ and $\left.-\left(\mathrm{CH}_{2}\right)_{3}\left(\mathrm{CH}_{2}\right)_{4}-\right), 0.94(\mathrm{~d}, \mathrm{~J}=6.6 \mathrm{~Hz}, 12 \mathrm{H}$, $\left.-\mathrm{CH}_{2} \mathrm{CH}\left(\mathrm{CH}_{3}\right) \mathrm{CH}_{2}-\right)$, 0.88-0.81 (m, 30H, $-\mathrm{CH}\left(\mathrm{CH}_{3}\right)_{2}$ and $\left.-\mathrm{CH}_{2} \mathrm{CH}_{3}\right) .{ }^{13} \mathrm{C} \mathrm{NMR}\left(125 \mathrm{MHz}, \mathrm{CDCl}_{3}, \delta\right)$ : 163.25, 153.18, 138.10, 134.44, 132.25, 131.50, 123.28, 123.01, 108.52, 73.54, 67.67, 39.44, 37.55, 36.66, $32.04,30.48,29.98,29.69,29.49,28.14,26.26,24.89,22.85,22.81,22.74,19.78,14.22$. MALDI-TOF MS $m / z$ of $[\mathrm{M}+\mathrm{Na}]^{+}$calculated for $\mathrm{C}_{94} \mathrm{H}_{134} \mathrm{~N}_{2} \mathrm{O}_{10}$ : 1474.0; Found: 1474.8 .
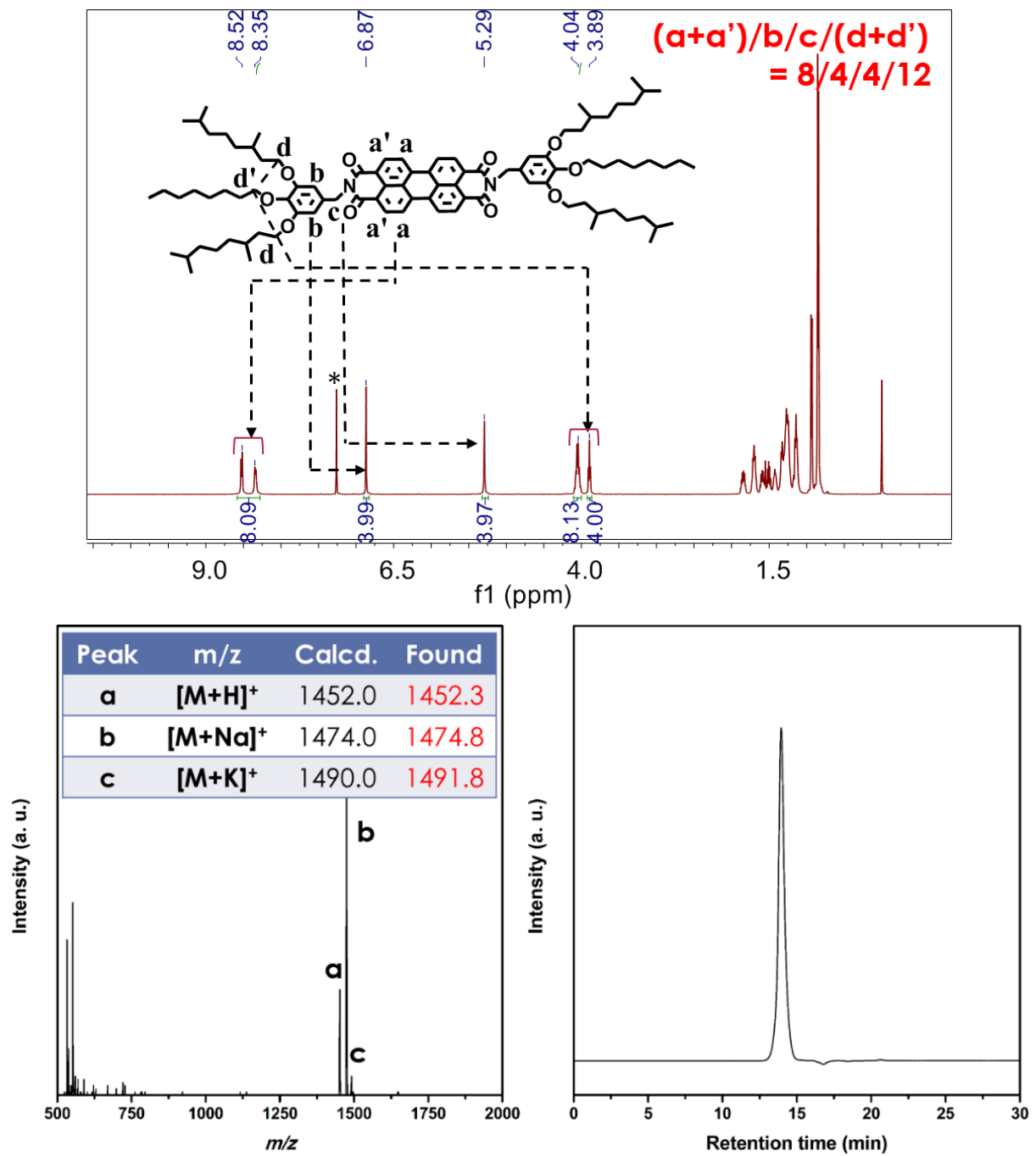

Figure S17. ${ }^{1} \mathrm{H}$ NMR spectra (top), MALDI-TOF MS spectra (bottom left) and HPLC trace (bottom right) of r8r-PBI. Target molecule r8r-PBI has been successfully synthesized. 


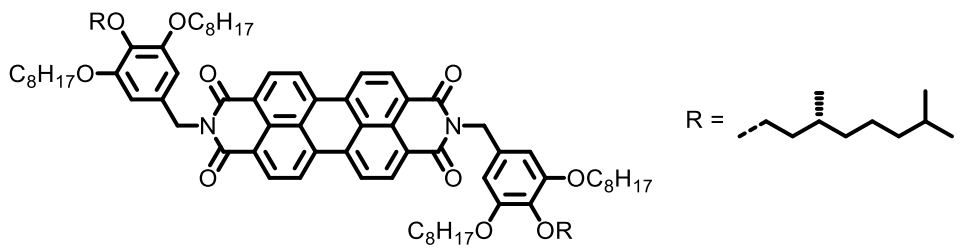

8S8-PBI. From 12-8S8 (0.94 g, $1.8 \mathrm{mmol})$, PTCDA $(0.32 \mathrm{~g}, 0.81 \mathrm{mmol})$ and $\mathrm{Zn}(\mathrm{OAc})_{2} .2 \mathrm{H}_{2} \mathrm{O}(0.18 \mathrm{~g}$, $0.81 \mathrm{mmol})$ in quinoline $(12 \mathrm{~mL}), \mathbf{8 S 8}-\mathbf{P B I}$ was obtained as red powder. Product: $0.93 \mathrm{~g}$. Yield: $82 \%$. Purity by HPLC: $99+\%$.

${ }^{1} \mathrm{H}$ NMR (400 MHz, $\mathrm{CDCl}_{3}, \delta$ ): 8.57 (d, $J=8.0 \mathrm{~Hz}, 4 \mathrm{H}, \mathrm{PBI}, 1,6,7,12$-positions), 8.41 (d, $J=8.1 \mathrm{~Hz}, 4 \mathrm{H}$, PBI, 2,5,8,11-positions), 6.85 (s, 4H, $\mathrm{Ar} H$ of dendrons), 5.29 (s, 4H, $-\mathrm{NCH}_{2}-$ ), 4.01 (t, $J=6.5 \mathrm{~Hz}, 8 \mathrm{H},-$ $\left.\mathrm{OCH}_{2} \mathrm{CH}_{2} \mathrm{CH}_{2}-\right), 4.00-3.88\left(\mathrm{~m}, 4 \mathrm{H},-\mathrm{OCH}_{2} \mathrm{CH}_{2} \mathrm{CH}\left(\mathrm{CH}_{3}\right)-\right), 1.86-1.73\left(\mathrm{~m}, 10 \mathrm{H},-\mathrm{CH}\left(\mathrm{CH}_{3}\right)_{2}\right.$ and $\left.\mathrm{OCH}_{2} \mathrm{CH}_{2} \mathrm{CH}_{2}-\right)$, 1.73-1.60 (m, $\left.2 \mathrm{H},-\mathrm{CH}\left(\mathrm{CH}_{3}\right) \mathrm{CH}_{2}-\right), 1.55-1.40\left(\mathrm{~m}, 12 \mathrm{H},-\mathrm{O}\left(\mathrm{CH}_{2}\right)_{2} \mathrm{CH}_{2}-\right.$ and $\left.\mathrm{OCH}_{2} \mathrm{CH}_{2} \mathrm{CH}\left(\mathrm{CH}_{3}\right)^{-}\right), 1.39-1.02\left(\mathrm{~m}, 44 \mathrm{H},-\mathrm{CH}\left(\mathrm{CH}_{3}\right)\left(\mathrm{CH}_{2}\right)_{3}-\right.$ and $\left.-\left(\mathrm{CH}_{2}\right)_{3}\left(\mathrm{CH}_{2}\right)_{4}-\right), 0.91-0.80(\mathrm{~m}, 30 \mathrm{H}$, $\left.{ }^{-} \mathrm{CH}_{3}\right) .{ }^{13} \mathrm{C} \mathrm{NMR}\left(125 \mathrm{MHz}, \mathrm{CDCl}_{3}, \delta\right): 163.28,153.20,144.18,143.14,140.49,138.09,134.48,131.53$, 123.29, 123.04, 108.49, 71.83, 69.35, 39.50, 37.66, 37.51, 32.00, 29.88, 29.63, 29.59, 29.46, 28.11, 26.31, 24.84, 22.82, 22.73, 19.70, 14.25. MALDI-TOF MS $m / z$ of $[\mathrm{M}+\mathrm{Na}]^{+}$calculated for $\mathrm{C}_{90} \mathrm{H}_{126} \mathrm{~N}_{2} \mathrm{O}_{10}$ : 1417.9; Found: 1419.1.
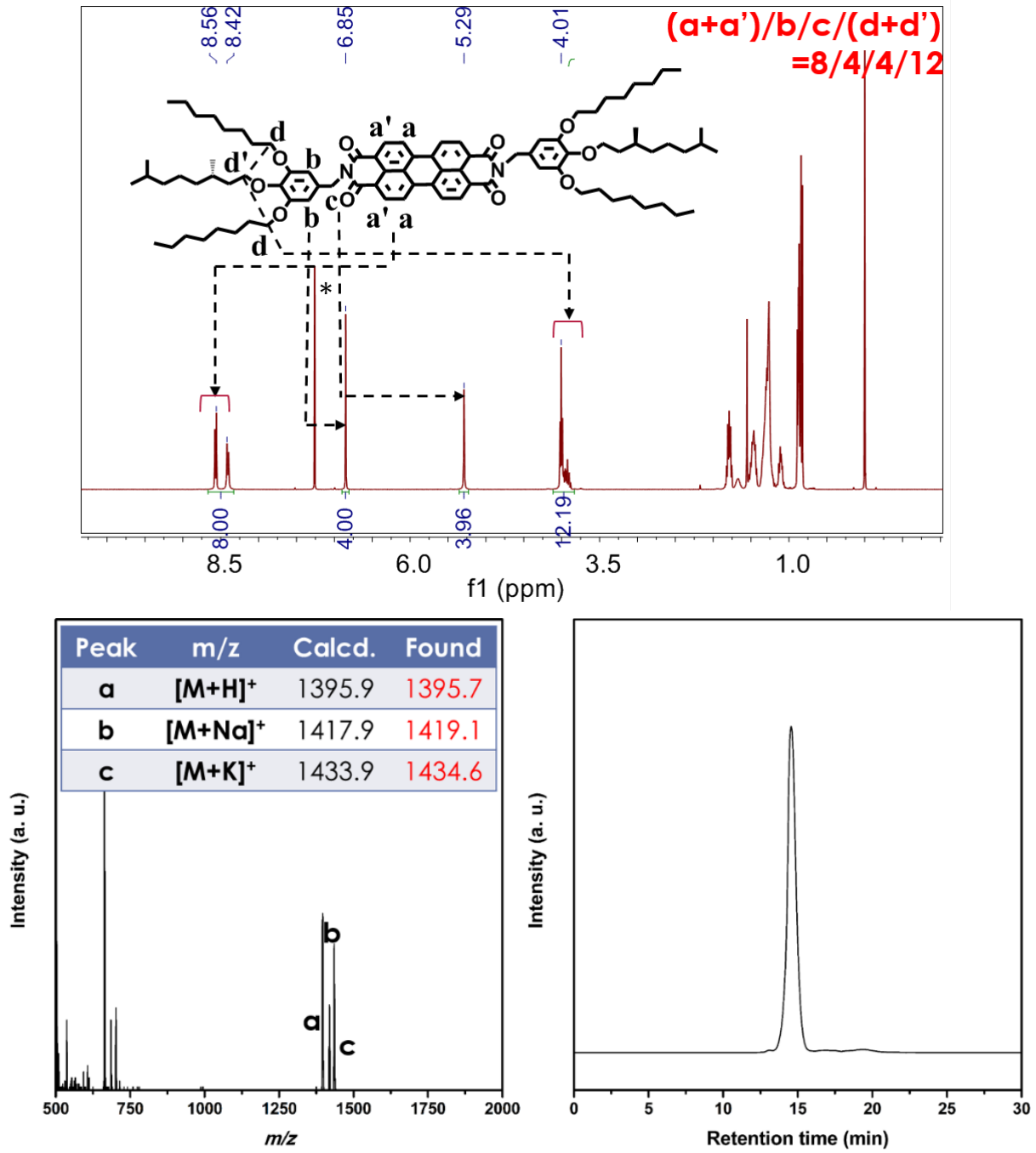

Figure S18. ${ }^{1} \mathrm{H}$ NMR spectra (top), MALDI-TOF MS spectra (bottom left) and HPLC trace (bottom right) of 8S8-PBI. Target molecule 8S8-PBI has been successfully synthesized. 


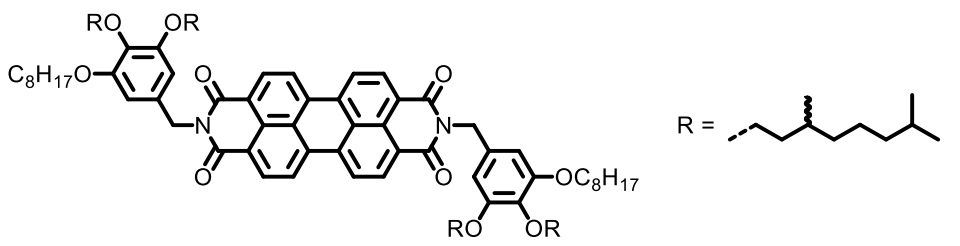

rr8-PBI. From 12-rr8 (0.66 g, $1.2 \mathrm{mmol})$, PTCDA $(0.21 \mathrm{~g}, 0.54 \mathrm{mmol})$ and $\mathrm{Zn}(\mathrm{OAc})_{2} .2 \mathrm{H}_{2} \mathrm{O}(0.12 \mathrm{~g}, 0.54$ $\mathrm{mmol}$ ) in quinoline $(8 \mathrm{~mL})$, rr8-PBI was obtained as red powder. Product: $0.55 \mathrm{~g}$. Yield: $63 \%$. Purity by HPLC: $99+\%$.

${ }^{1} \mathrm{H}$ NMR (500 MHz, $\left.\mathrm{CDCl}_{3}, \delta\right): 8.58$ (d, $J=7.9 \mathrm{~Hz}, 4 \mathrm{H}, \mathrm{PBI}, 1,6,7,12$-positions), 8.43 (d, $J=8.1 \mathrm{~Hz}, 4 \mathrm{H}$, PBI, 2,5,8,11-positions), 6.85 (overlapping s, $4 \mathrm{H}, \mathrm{Ar} H$ of dendrons), 5.29 (s, $4 \mathrm{H},-\mathrm{NCH}_{2}-$ ), 4.10-3.85 (m, $\left.12 \mathrm{H},-\mathrm{OCH}_{2}-\right), 1.89-1.74\left(\mathrm{~m}, 8 \mathrm{H},-\mathrm{OCH}_{2} \mathrm{CH}_{2} \mathrm{CH}_{2}-\right.$ and $\left.-\mathrm{CH}\left(\mathrm{CH}_{3}\right)_{2}\right), 1.74-1.63\left(\mathrm{~m}, 4 \mathrm{H},-\mathrm{CH}_{\left(\mathrm{CH}_{3}\right) \mathrm{CH}_{2}-}\right.$ ), $1.63-1.41\left(\mathrm{~m}, 12 \mathrm{H},-\mathrm{O}\left(\mathrm{CH}_{2}\right)_{2} \mathrm{CH}_{2}-\right.$ and $\left.-\mathrm{OCH}_{2} \mathrm{CH}_{2} \mathrm{CH}\left(\mathrm{CH}_{3}\right)-\right), 1.38-1.03\left(\mathrm{~m}, 40 \mathrm{H},-\mathrm{CH}\left(\mathrm{CH}_{3}\right)\left(\mathrm{CH}_{2}\right)_{3}-\right.$ and $\left.-\left(\mathrm{CH}_{2}\right)_{3}\left(\mathrm{CH}_{2}\right)_{4}-\right), 0.93\left(\mathrm{~d}, J=6.5 \mathrm{~Hz}, 6 \mathrm{H},-\mathrm{CH}_{2} \mathrm{CH}\left(\mathrm{CH}_{3}\right) \mathrm{CH}_{2}-\right), 0.90-0.79\left(\mathrm{~m}, 36 \mathrm{H},-\mathrm{CH}\left(\mathrm{CH}_{3}\right)_{2}\right.$ and $\left.-\mathrm{CH}_{2} \mathrm{CH}_{3}\right) .{ }^{13} \mathrm{C}$ NMR $\left(125 \mathrm{MHz}, \mathrm{CDCl}_{3}, \delta\right): 162.91,152.96,137.86,134.06,132.04,131.16,128.74$, 125.74, 122.94, 108.29, 71.59, 69.12, 67.44, 39.25, 39.21, 37.42, 37.35, 37.27, 36.41, 31.76, 29.76, 29.64, $29.40,29.35,29.22,27.89,27.86,26.08,24.66,24.59,22.62,22.58,22.51,22.48,19.55,19.52,19.47$, 14.00. MALDI-TOF MS $m / z$ of $[\mathrm{M}+\mathrm{Na}]^{+}$calculated for $\mathrm{C}_{94} \mathrm{H}_{134} \mathrm{~N}_{2} \mathrm{O}_{10}$ : 1474.0 ; Found: 1475.0 .
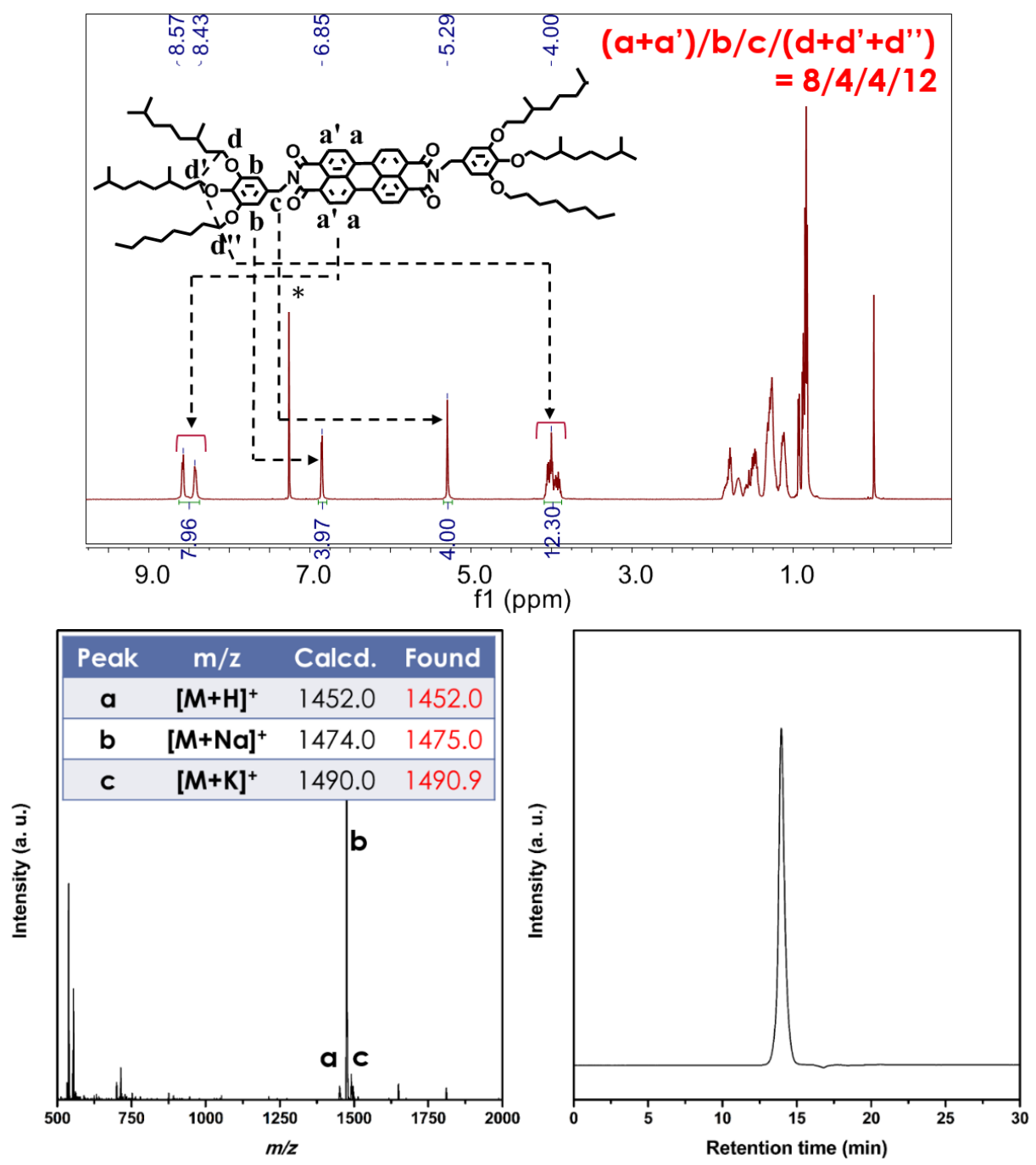

Figure S19. ${ }^{1}$ H NMR spectra (top), MALDI-TOF MS spectra (bottom left) and HPLC trace (bottom right) of rr8-PBI. Target molecule rr8-PBI has been successfully synthesized. 


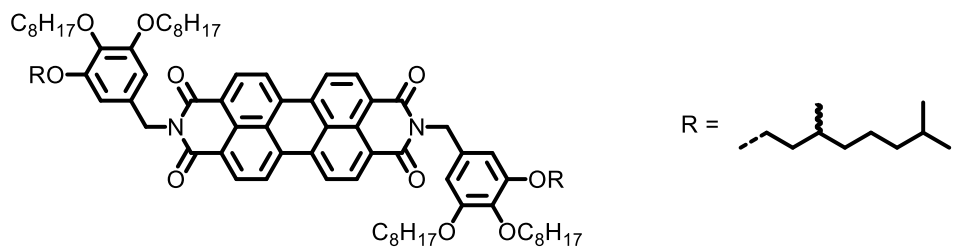

88r-PBI. From 12-88r (0.62 g, $1.2 \mathrm{mmol})$, PTCDA $(0.21 \mathrm{~g}, 0.54 \mathrm{mmol})$ and $\mathrm{Zn}(\mathrm{OAc})_{2} .2 \mathrm{H}_{2} \mathrm{O}(0.12 \mathrm{~g}$, $0.54 \mathrm{mmol})$ in quinoline $(8 \mathrm{~mL}), \mathbf{8 8 r}-\mathbf{P B I}$ was obtained as red powder. Product: $0.55 \mathrm{~g}$. Yield: $62 \%$. Purity by HPLC: $99+\%$.

${ }^{1} \mathrm{H}$ NMR $\left(500 \mathrm{MHz}, \mathrm{CDCl}_{3}, \delta\right): 8.55$ (d, $J=7.9 \mathrm{~Hz}, 4 \mathrm{H}, \mathrm{PBI}, 1,6,7,12$-positions), 8.38 (d, $J=8.1 \mathrm{~Hz}, 4 \mathrm{H}$, PBI, 2,5,8,11-positions), 6.86 (overlapping s, $4 \mathrm{H}, \mathrm{Ar} H$ of dendrons), 5.29 (s, $4 \mathrm{H},-\mathrm{NCH}_{2}-$ ), 4.10-3.97 (m, $8 \mathrm{H},-\mathrm{OCH}_{2} \mathrm{CH}_{2} \mathrm{CH}_{2}-$ and $\left.-\mathrm{OCH}_{2} \mathrm{CH}_{2} \mathrm{CH}(\mathrm{CH})_{3}-\right), 3.90\left(\mathrm{t}, J=6.6 \mathrm{~Hz}, 4 \mathrm{H},-\mathrm{OCH}_{2} \mathrm{CH}_{2} \mathrm{CH}_{2-}\right), 1.89-1.65$ $\left(\mathrm{m}, 10 \mathrm{H},-\mathrm{CH}\left(\mathrm{CH}_{3}\right)_{2}\right.$ and $\left.-\mathrm{OCH}_{2} \mathrm{CH}_{2} \mathrm{CH}_{2}-\right), 1.63-1.38\left(\mathrm{~m}, 14 \mathrm{H},-\mathrm{CH}\left(\mathrm{CH}_{3}\right) \mathrm{CH}_{2}-,-\mathrm{O}\left(\mathrm{CH}_{2}\right)_{2} \mathrm{CH}_{2}-\right.$ and $\left.\mathrm{OCH}_{2} \mathrm{CH}_{2} \mathrm{CH}\left(\mathrm{CH}_{3}\right)-\right), 1.38-1.08\left(\mathrm{~m}, 44 \mathrm{H},-\mathrm{CH}\left(\mathrm{CH}_{3}\right)\left(\mathrm{CH}_{2}\right)_{3}-\right.$ and $\left.-\left(\mathrm{CH}_{2}\right)_{3}\left(\mathrm{CH}_{2}\right)_{4}-\right), 0.93(\mathrm{~d}, J=6.6 \mathrm{~Hz}$, $\left.6 \mathrm{H},-\mathrm{CH}_{2} \mathrm{CH}\left(\mathrm{CH}_{3}\right) \mathrm{CH}_{2}-\right), 0.90-0.80\left(\mathrm{~m}, 24 \mathrm{H},-\mathrm{CH}\left(\mathrm{CH}_{3}\right)_{2}\right.$ and $\left.-\mathrm{CH}_{2} \mathrm{CH}_{3}\right) .{ }^{13} \mathrm{C} \mathrm{NMR}\left(125 \mathrm{MHz}, \mathrm{CDCl}_{3}\right.$,

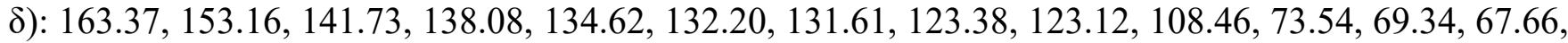
$39.43,37.54,36.64,32.03,31.99,30.47,29.97,28.13,26.29,26.25,24.88,22.85,22.81,22.74,19.77$, 14.22. MALDI-TOF MS $m / z$ of [M $+\mathrm{Na}]^{+}$calculated for $\mathrm{C}_{90} \mathrm{H}_{126} \mathrm{~N}_{2} \mathrm{O}_{10}$ : 1417.9; Found: 1418.8 .
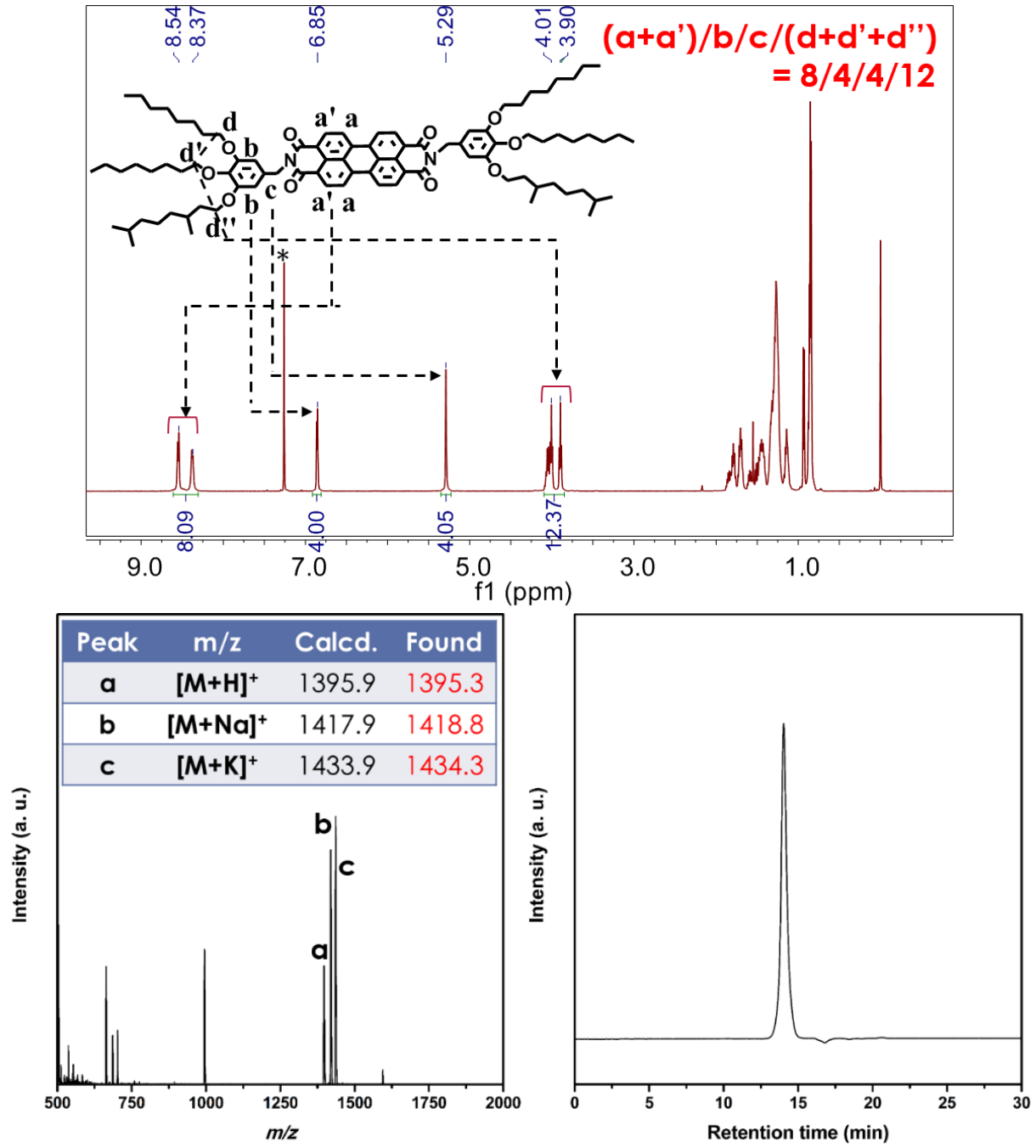

Figure S20. ${ }^{1} \mathrm{H}$ NMR spectra (top), MALDI-TOF MS spectra (bottom left) and HPLC trace (bottom right) of 88r-PBI. Target molecule 88r-PBI has been successfully synthesized. 
4. Thermal Analysis of Linear-Racemic Hybrid PBIs
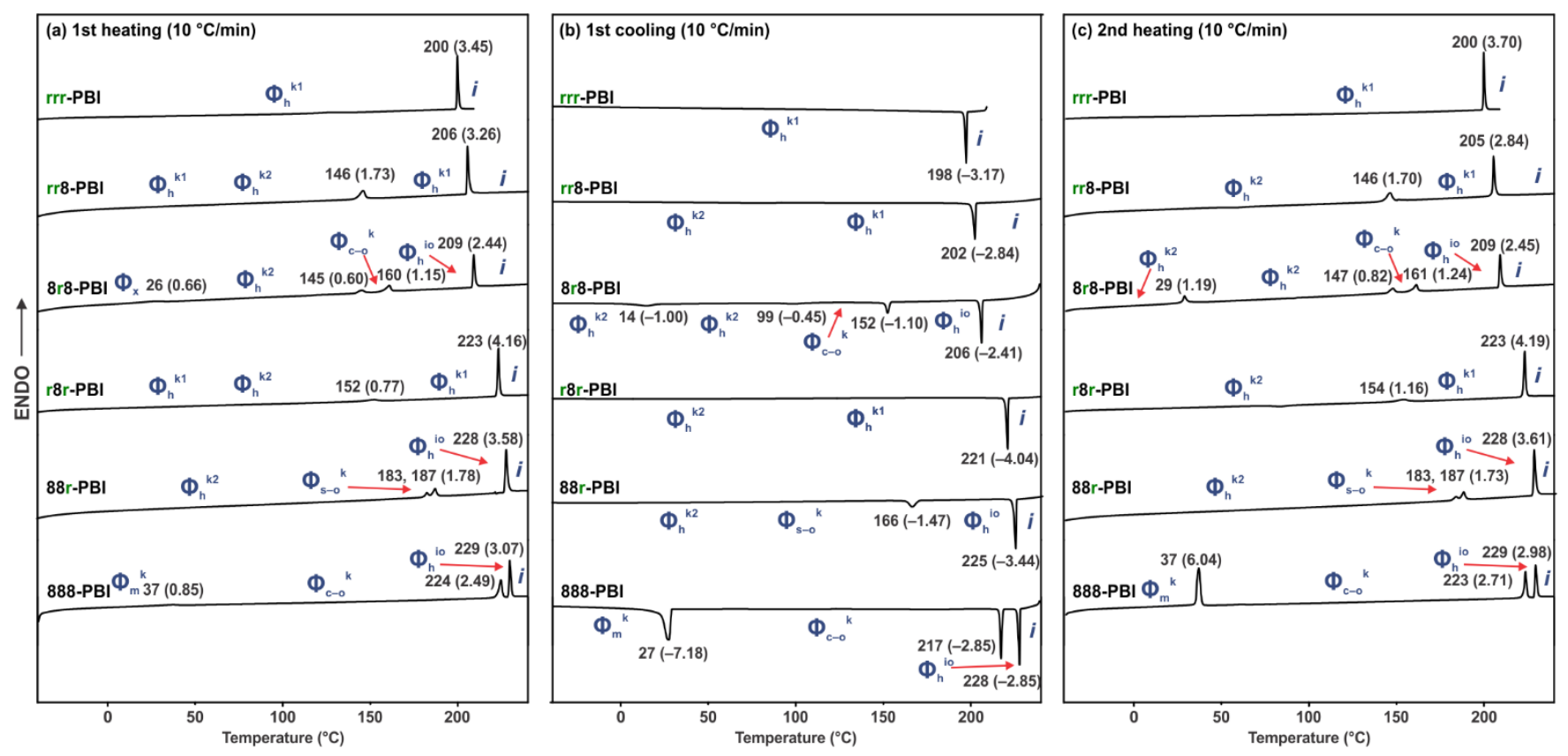

Figure S21. DSC traces of PBIs with hybrid dm $8 * / \mathrm{C} 8$ dendrons. a) First heating, (b) first cooling, and (c) second heating scans recorded with heating and cooling rates of $10^{\circ} \mathrm{C} / \mathrm{min}$. Phases determined by X-ray diffraction (to be discussed later), transition temperatures (in ${ }^{\circ} \mathrm{C}$ ), and associated enthalpy changes (in parentheses, in $\mathrm{kcal} / \mathrm{mol}$ ) are indicated. Phase notation: $\boldsymbol{\Phi}_{\mathbf{h}}{ }^{\mathbf{k}}{ }^{-}$columnar hexagonal crystalline phase with offset dimers ${ }^{31} ; \boldsymbol{\Phi}_{\mathbf{h}}{ }^{\mathbf{k} 2}$ - columnar hexagonal crystalline phase with cogwheel assembly ${ }^{31} ; \boldsymbol{\Phi}_{\mathbf{x}}-$ unknown columnar phase; $\boldsymbol{\Phi}_{\mathbf{c}-\mathbf{0}}{ }^{\mathbf{k}}$ - columnar centered orthorhombic crystalline phase; $\boldsymbol{\Phi}_{\mathbf{m}}{ }^{\mathbf{k}}-$ columnar monoclinic crystalline phase; $\boldsymbol{\Phi}_{\mathbf{h}}{ }^{\text {io }}-2 \mathrm{D}$ columnar hexagonal phase with short range intracolumnar order; $\boldsymbol{i}$ - isotropic. Note: quantitative uncertainties are $\pm 1{ }^{\circ} \mathrm{C}$ for thermal transition temperatures and $\sim 2 \%$ for the associated enthalpy changes reported in $\mathrm{kcal} / \mathrm{mol}$. 
Table ST1. Transition Temperatures and Associated Enthalpy Changes of Sequence-Defined Hybrid Linear-Racemic PBIs and Linear-Chiral PBIs Determined by DSC and Phases Determined by XRD

\begin{tabular}{|c|c|c|}
\hline & \multicolumn{2}{|c|}{ Thermal transition temperature $\left({ }^{\circ} \mathrm{C}\right)$ and corresponding enthalpy change $(\mathrm{kcal} / \mathrm{mol})$} \\
\hline \multicolumn{3}{|c|}{ Second heating } \\
\hline rrr-PBI & $\begin{array}{l}\Phi_{\mathbf{h}}{ }^{\mathbf{k} 1} 200(3.45) \boldsymbol{i} \\
\Phi_{\mathbf{h}}{ }^{\mathbf{k} 1} 200(3.70) \boldsymbol{i}\end{array}$ & $i 198(-3.17) \Phi_{\mathbf{h}}{ }^{k 1}$ \\
\hline rr8-PBI & $\begin{array}{l}\boldsymbol{\Phi}_{\mathbf{h}}{ }^{\mathbf{k} 1}-{ }^{a} \boldsymbol{\Phi}_{\mathbf{h}}{ }^{\mathbf{k} 2} 146(1.73) \boldsymbol{\Phi}_{\mathbf{h}}{ }^{\mathbf{k} 1} 206(3.26) \boldsymbol{i} \\
\boldsymbol{\Phi}_{\mathbf{h}}{ }^{\mathbf{2}} 146(1.70) \boldsymbol{\Phi}_{\mathbf{h}}{ }^{\mathbf{k} 1} 205(2.84) \boldsymbol{i}\end{array}$ & $\boldsymbol{i} 202(-2.84) \boldsymbol{\Phi}_{\mathbf{h}}{ }^{\mathbf{k} 1}-{ }^{a} \boldsymbol{\Phi}_{\mathbf{h}}{ }^{\mathbf{k} 2}$ \\
\hline 8r8-PBI & 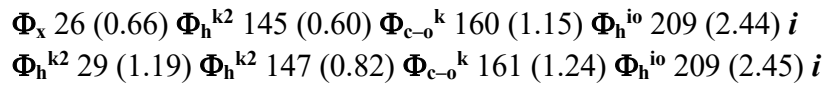 & $\begin{array}{l}\boldsymbol{i} 206(-2.41) \boldsymbol{\Phi}_{\mathbf{h}}^{\text {io }} 152(-1.10) \boldsymbol{\Phi}_{\mathbf{c - 0}}{ }^{\mathbf{k}} 99(-0.45) \\
\boldsymbol{\Phi}_{\mathbf{h}}{ }^{\mathbf{k}} 14(-1.00) \boldsymbol{\Phi}_{\mathbf{h}}{ }^{\mathbf{k} 2}\end{array}$ \\
\hline r8r-PBI & $\begin{array}{l}\boldsymbol{\Phi}_{\mathbf{h}}{ }^{\mathbf{1} 1}-{ }^{a} \boldsymbol{\Phi}_{\mathbf{h}}{ }^{\mathbf{k} 2} 152(0.77) \boldsymbol{\Phi}_{\mathbf{h}}{ }^{\mathbf{k} 1} 223(4.16) \boldsymbol{i} \\
\boldsymbol{\Phi}_{\mathbf{h}}{ }^{\mathbf{2} 2} 154(1.16) \boldsymbol{\Phi}_{\mathbf{h}}{ }^{\mathbf{k} 1} 223(4.19) \boldsymbol{i}\end{array}$ & $\boldsymbol{i} 221(-4.04) \boldsymbol{\Phi}_{\mathbf{h}}{ }^{\mathbf{k} 1}-{ }^{a} \boldsymbol{\Phi}_{\mathbf{h}}{ }^{\mathbf{k} 2}$ \\
\hline 88r-PBI & $\begin{array}{l}\boldsymbol{\Phi}_{\mathbf{h}}{ }^{\mathbf{k} 2}-{ }^{a} \boldsymbol{\Phi}_{\mathrm{s}-\mathbf{0}}{ }^{\mathbf{k}} 183,187(1.78) \boldsymbol{\Phi}_{\mathbf{h}}{ }^{\mathrm{io}} 228(3.58) \boldsymbol{i} \\
\boldsymbol{\Phi}_{\mathbf{h}}{ }^{\mathbf{k} 2}{ }^{a} \boldsymbol{\Phi}_{\mathbf{s - 0}}{ }^{\mathbf{k}} 183,187(1.73) \boldsymbol{\Phi}_{\mathbf{h}}{ }^{\text {io }} 228(3.61) \boldsymbol{i}\end{array}$ & $\boldsymbol{i} 225(-3.44) \boldsymbol{\Phi}_{\mathbf{h}}{ }^{\mathrm{io}} 166(-1.47) \boldsymbol{\Phi}_{\mathrm{s}-\mathbf{0}}{ }^{\mathbf{k}}-{ }^{a} \boldsymbol{\Phi}_{\mathbf{h}}{ }^{\mathbf{k} 2}$ \\
\hline $888-\mathrm{PBI}{ }^{b}$ & 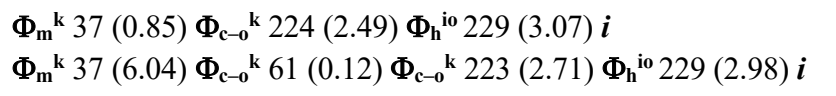 & $\begin{array}{l}\boldsymbol{i} 228(-2.85) \boldsymbol{\Phi}_{\mathbf{h}}^{\mathrm{io}} 217(-2.85) \boldsymbol{\Phi}_{\mathbf{c}-\mathbf{0}}^{\mathrm{k}} 27(-7.18) \\
\boldsymbol{\Phi}_{\mathbf{m}}{ }^{\mathbf{k}}\end{array}$ \\
\hline rr10-PBI & $\begin{array}{l}\boldsymbol{\Phi}_{\mathbf{h}}{ }^{\mathbf{k} 2} 111(0.31) \boldsymbol{\Phi}_{\mathbf{h}}^{\text {io }} 204(3.26) \boldsymbol{i} \\
\boldsymbol{\Phi}_{\mathbf{h}}{ }^{\mathbf{k} 2} 118(0.38) \boldsymbol{\Phi}_{\mathbf{h}}^{\text {io }} 204(3.15) \boldsymbol{i}\end{array}$ & $i 201(-3.15) \Phi_{\mathbf{h}}{ }^{\text {io }}$ \\
\hline 8S8-PBI & 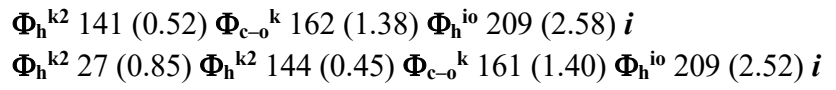 & $\begin{array}{l}\boldsymbol{i} 206(-2.30) \boldsymbol{\Phi}_{\mathbf{h}}^{\mathrm{io}} 152(-1.09) \boldsymbol{\Phi}_{\mathbf{c}-\mathbf{0}}^{\mathbf{k}} 89(-0.58) \\
\boldsymbol{\Phi}_{\mathbf{h}}{ }^{\mathbf{k}} 11(-0.67) \boldsymbol{\Phi}_{\mathbf{h}}^{\mathbf{k} 2}\end{array}$ \\
\hline S8S-PBI & $\begin{array}{l}\boldsymbol{\Phi}_{\mathbf{h}}{ }^{\mathbf{k} 2} 143(0.78) \Phi_{\mathbf{h}}{ }^{\mathbf{k} 1} 224(4.07) \boldsymbol{i} \\
\boldsymbol{\Phi}_{\mathbf{h}}{ }^{\mathbf{2} 2} 147(1.00) \Phi_{\mathbf{h}}{ }^{\mathbf{k} 1} 224(4.06) \boldsymbol{i}\end{array}$ & $\boldsymbol{i} 221(-4.02) \boldsymbol{\Phi}_{\mathbf{h}}{ }^{\mathbf{k} 1}-{ }^{a} \boldsymbol{\Phi}_{\mathbf{h}} \mathbf{k 2}$ \\
\hline $\begin{array}{l}{ }^{a} \text { No first order } \\
\text { offset dimers }{ }^{10} \\
\text { centered orthor }\end{array}$ & $\begin{array}{l}\text { se transition observed by DSC. }{ }^{b} \text { Data taken from ref. }{ }^{1} . \text { Phase no } \\
\mathbf{k} 2-\text { columnar hexagonal crystalline phase with cogwheel assem } \\
\text { abic crystalline phase; } \boldsymbol{\Phi}_{\mathbf{m}} \mathbf{k}^{\mathbf{k}}-\text { columnar monoclinic crystalline } \\
\text { ar order; } \boldsymbol{i} \text { - isotropic. Note: quantitative uncertainties are } \pm 1 \\
\text { changes reported in kcal } / \mathrm{mol} \text {. }\end{array}$ & 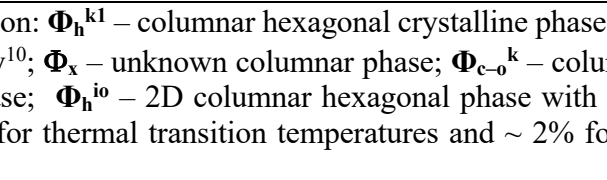 \\
\hline
\end{tabular}


5. Structural and Retrostructural Analysis of Linear-Racemic Hybrid PBIs

(a)

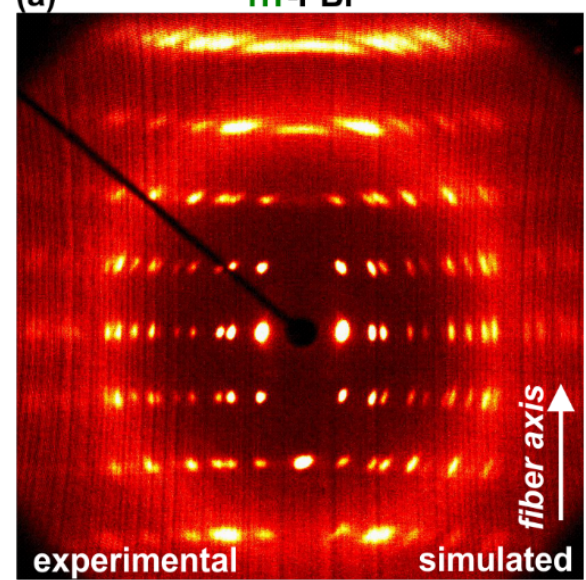

$\Phi_{\mathrm{h}}{ }^{\mathrm{k} 2}, \mathrm{~T}=100^{\circ} \mathrm{C}$

$a=b=27.0 \AA, c=14.7 \AA$

(d)

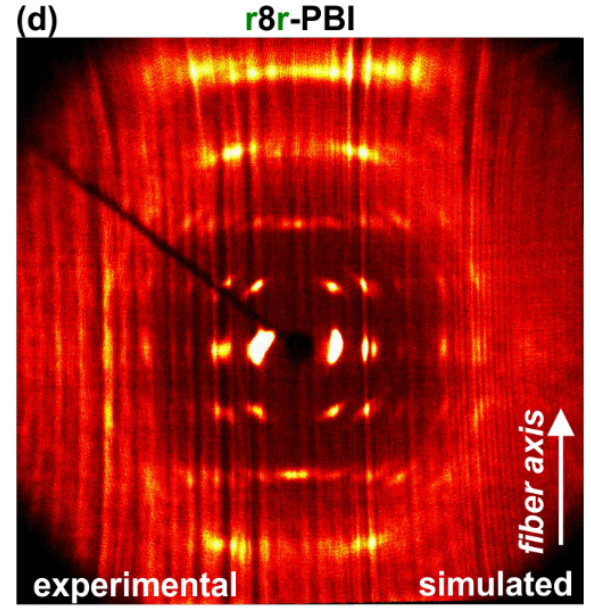

$\Phi_{\mathrm{h}}{ }^{\mathrm{k} 2}, \mathrm{~T}=100^{\circ} \mathrm{C}$

$a=b=26.9 \AA, c=14.9 \AA$ (b)

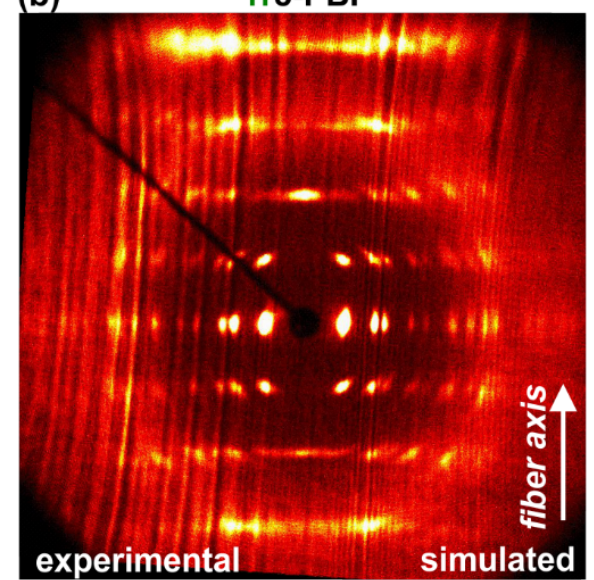

$\Phi_{\mathrm{h}}^{\mathrm{k} 2}, \mathrm{~T}=100{ }^{\circ} \mathrm{C}$

$a=b=26.4 \AA, c=14.8 \AA$

(e)

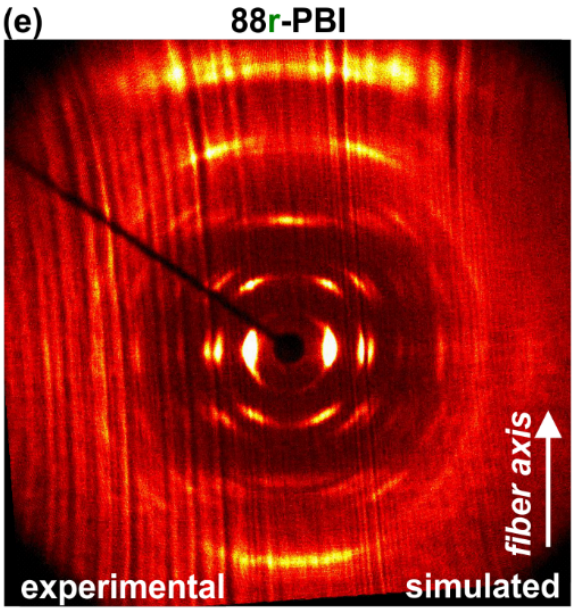

$\Phi_{\mathrm{h}}{ }^{\mathrm{k} 2}, \mathrm{~T}=100^{\circ} \mathrm{C}$

$a=b=26.3 \AA, c=14.8 \AA$ (c)

8r8-PBI

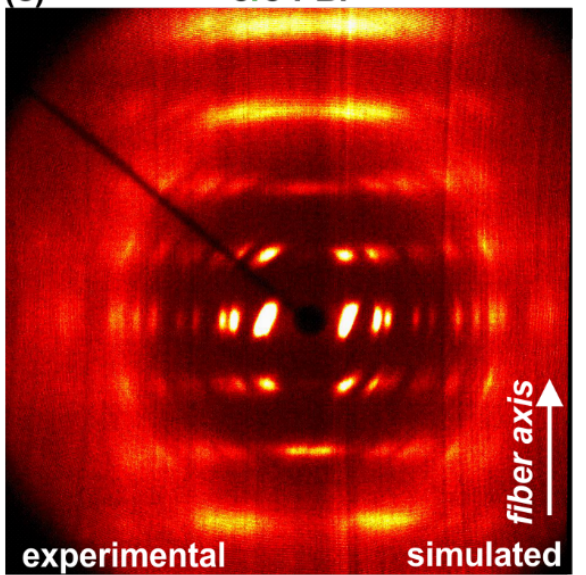

$\Phi_{\mathrm{h}}{ }^{\mathrm{k} 2}, \mathrm{~T}=100^{\circ} \mathrm{C}$

$a=b=27.0 \AA, c=14.8 \AA$

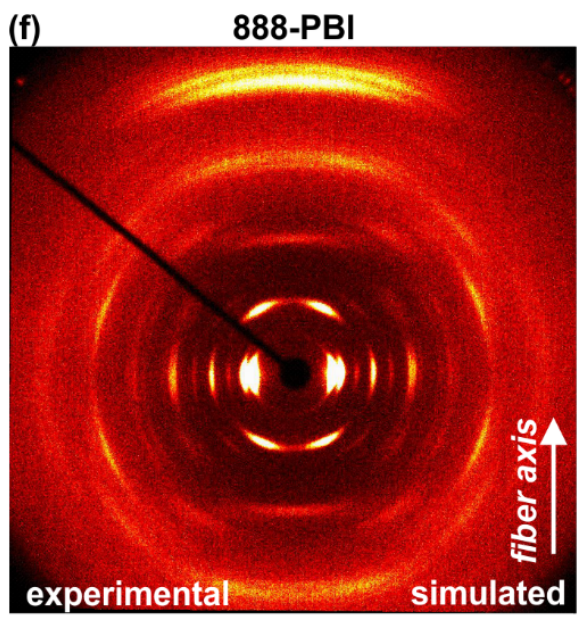

$\Phi_{\mathrm{c}-\mathrm{O}}{ }^{\mathrm{k}}, \mathrm{T}=80^{\circ} \mathrm{C}$

$a=37.6 \AA, b=30.5 \AA, c=14.5 \AA$

Figure S22. Experimental oriented fiber X-ray diffraction (XRD) of (a) rrr-PBI, (b) rr8-PBI, (c) r8rPBI, (d) 8r8-PBI, (e) 88r-PBI, and (f) 888-PBI. Fiber axis, temperature, phase, and lattice parameters are indicated. rrr-PBI (a) was annealed at $100{ }^{\circ} \mathrm{C}$ for $3 \mathrm{~h}$ before measurement. All other samples were measured with freshly extruded fibers. 
Table ST2. Structural Analysis of Sequence-Defined Hybrid PBIs by XRD

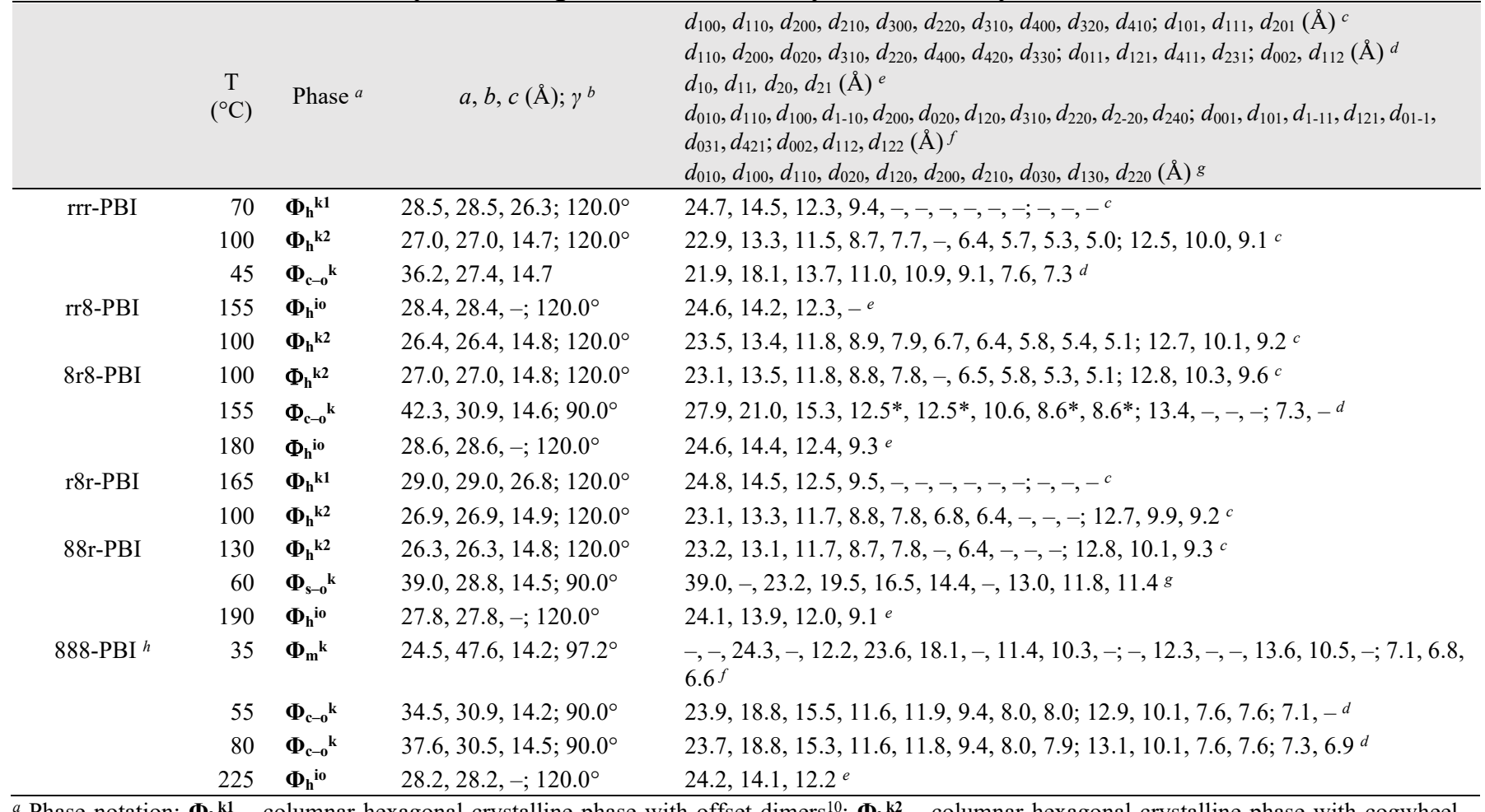

${ }^{a}$ Phase notation: $\boldsymbol{\Phi}_{\mathbf{h}}{ }^{\mathbf{k} 1}-$ columnar hexagonal crystalline phase with offset dimers ${ }^{10} ; \boldsymbol{\Phi}_{\mathbf{h}}{ }^{\mathbf{k} 2}-$ columnar hexagonal crystalline phase with cogwheel assembly ${ }^{10} ; \boldsymbol{\Phi}_{\mathbf{x}}{ }^{\mathbf{k}}$ - unknown crystalline columnar phase; $\boldsymbol{\Phi}_{\mathbf{h}}{ }^{\mathbf{i o}}-2 \mathrm{D}$ columnar hexagonal phase with short range intracolumnar order; $\boldsymbol{\Phi}_{\mathbf{c}-\mathbf{0}}{ }^{\mathbf{k}}-$ columnar centered orthorhombic crystalline phase; $\boldsymbol{\Phi}_{\mathbf{s}-\mathbf{0}} \mathbf{k}-$ columnar simple orthorhombic crystalline phase; $\boldsymbol{\Phi}_{\mathbf{m}}{ }^{\mathbf{k}}-$ columnar monoclinic crystalline phase. ${ }^{b}$ Lattice parameters determined from fiber X-ray diffraction as follows: for hexagonal phases, $d_{\mathrm{hkl}}=\left[4\left(h^{2}+k^{2}+h k\right) /\left(3 a^{2}\right)+(l / c)^{2}\right]^{-1 / 2}$; for orthorhombic phases, $d_{\mathrm{hkl}}=\left[(h / a)^{2}+(k / b)^{2}+(l / c)^{2}\right]^{-1 / 2}$; for monoclinic phases: $d_{\mathrm{hkl}}=\left[(h / a \sin \gamma)^{2}+(k / b \sin \gamma)^{2}+(l / c)^{2}-\left\{(2 h k \cos \gamma) /\left(a b \sin ^{2} \gamma\right)\right\}\right]^{-1 / 2}{ }^{c}$ Experimental diffraction peak $d$-spacings for the $\boldsymbol{\Phi}_{\mathbf{h}}{ }^{\mathbf{k}}$ and $\boldsymbol{\Phi}_{\mathbf{h}}{ }^{\mathbf{k} 2}$ phases. ${ }^{d}$ Experimental diffraction peak $d$-spacings for the $\boldsymbol{\Phi}_{\mathbf{c}-\mathbf{0}}{ }^{\mathbf{k}}$ phase. ${ }^{e}$ Experimental diffraction peak $d$-spacings for the $\boldsymbol{\Phi}_{\mathbf{h}}{ }^{\text {io }}$ phase. ${ }^{f}$ Experimental diffraction peak $d$-spacings for the $\boldsymbol{\Phi}_{\mathbf{m}}{ }^{\mathbf{k}}$ phase. ${ }^{g}$ Experimental diffraction peak $d$-spacings for the $\boldsymbol{\Phi}_{\mathbf{s}-\mathbf{0}}{ }^{\mathbf{k}}$ phase. ${ }^{h}$ Data taken from ref. ${ }^{1} .{ }^{*}$ indicates overlapping features in XRD pattern. 
Table ST3. Retrostructural Analysis of Sequence-Defined Hybrid Linear-Racemic PBIs

\begin{tabular}{|c|c|c|c|c|c|c|c|c|c|}
\hline & $\begin{array}{c}\mathrm{T} \\
\left({ }^{\circ} \mathrm{C}\right) \\
\end{array}$ & Phase $^{a}$ & $a, b, c(\AA)^{b}$ & $\alpha, \beta, \gamma\left({ }^{\circ}\right)^{c}$ & $\mathrm{D}_{\mathrm{col}}(\AA)^{d}$ & $t(\AA)^{e}$ & $\begin{array}{c}\rho^{f} \\
\left(\mathrm{~g} / \mathrm{cm}^{3}\right)\end{array}$ & $\begin{array}{c}\mathrm{M}_{\mathrm{w}}{ }^{g} \\
(\mathrm{~g} / \mathrm{mol})\end{array}$ & $\mu^{h}$ \\
\hline \multirow[t]{2}{*}{ rrr-PBI } & 70 & $\Phi_{\mathrm{h}}{ }^{\mathrm{k} 1}$ & $28.5,28.5,26.3$ & $90.0,90.0,120.0$ & 28.5 & 3.3 & 1.05 & 1508.22 & $0.97 \approx 1$ \\
\hline & 100 & $\Phi_{h^{2}}{ }^{k 2}$ & $27.0,27.0,14.7$ & $90.0,90.0,120.0$ & 27.0 & 3.7 & & & $0.97 \approx 1$ \\
\hline \multirow[t]{2}{*}{ rr8-PBI } & 155 & $\Phi_{h}{ }_{h}^{\text {io }}$ & $28.4,28.4,-$ &,,-- 120.0 & 28.4 & 3.3 & 1.05 & 1452.11 & $1.00 \approx 1$ \\
\hline & 100 & $\Phi_{\mathrm{h}}{ }^{\mathrm{k} 2}$ & $26.4,26.4,14.8$ & $90.0,90.0,120.0$ & 26.4 & 3.7 & & & $0.97 \approx 1$ \\
\hline \multirow[t]{3}{*}{ 8r8-PBI } & 100 & $\Phi_{\mathrm{h}}^{\mathrm{k} 2}$ & $27.0,27.0,14.8$ & $90.0,90.0,120.0$ & 27.0 & 3.7 & 1.07 & 1396.00 & $1.08 \approx 1$ \\
\hline & 155 & $\Phi_{c-0}^{k}$ & $42.3,30.9,14.6$ & $90.0,90.0,90.0$ & 26.2 & 3.7 & & & $1.10 \approx 1$ \\
\hline & 180 & $\Phi_{h}{ }^{\text {io }}$ & $28.6,28.6,-$ &,,-- 120.0 & 28.6 & 3.4 & & & $1.11 \approx 1$ \\
\hline \multirow[t]{2}{*}{ r8r-PBI } & 165 & $\Phi_{\mathrm{h}}{ }^{\mathrm{k} 1}$ & $29.0,29.0,26.8$ & $90.0,90.0,120.0$ & 29.0 & 3.4 & 1.06 & 1452.11 & $1.07 \approx 1$ \\
\hline & 100 & $\Phi_{\mathrm{h}}^{\mathrm{k} 2}$ & $26.9,26.9,14.9$ & $90.0,90.0,120.0$ & 26.9 & 3.4 & & & $1.03 \approx 1$ \\
\hline \multirow[t]{3}{*}{ 88r-PBI } & 130 & $\Phi_{\mathrm{h}} \mathrm{k}^{2}$ & $26.3,26.3,14.8$ & $90.0,90.0,120.0$ & 26.3 & 3.7 & 1.07 & 1396.00 & $1.02 \approx 1$ \\
\hline & 60 & $\Phi_{s-0}^{k}$ & $39.0,28.8,14.5$ & $90.0,90.0,90.0$ & 24.2 & 3.6 & & & $0.94 \approx 1$ \\
\hline & 190 & $\Phi_{h}^{\text {io }}$ & $27.8,27.8,-$ &,,-- 120.0 & 27.8 & 3.4 & & & $1.05 \approx 1$ \\
\hline \multirow[t]{4}{*}{$888-\mathrm{PBI}^{i}$} & 35 & $\Phi_{\mathrm{m}}{ }^{\mathrm{k}}$ & $47.6,24.5,14.2$ & $90.0,90.0,97.2$ & 24.5 & 3.6 & 1.08 & 1339.89 & $1.00 \approx 1$ \\
\hline & 55 & $\Phi_{c-0}^{k}$ & $37.5,30.9,14.2$ & $90.0,90.0,90.0$ & 23.9 & 3.6 & & & $1.00 \approx 1$ \\
\hline & 80 & $\Phi_{c-0} k$ & $37.6,30.5,14.5$ & $90.0,90.0,90.0$ & 24.2 & 3.6 & & & $1.01 \approx 1$ \\
\hline & 225 & $\Phi_{h}^{\text {io }}$ & $28.2,28.2,-$ &,,-- 120.0 & 28.2 & 3.5 & & & $1.17 \approx 1$ \\
\hline
\end{tabular}

${ }^{a}$ Phase notation: $\boldsymbol{\Phi}_{\mathbf{h}}{ }^{\mathbf{k} 1}$ - columnar hexagonal crystalline phase with offset dimers ${ }^{10} ; \boldsymbol{\Phi}_{\mathbf{h}}{ }^{\mathbf{k} 2}-$ columnar hexagonal crystalline phase with cogwheel assembly ${ }^{10} ; \boldsymbol{\Phi}_{\mathbf{c}-\mathbf{0}}{ }^{\mathbf{k}}-$ columnar centered orthorhombic crystalline phase; $\boldsymbol{\Phi}_{\mathbf{h}}{ }^{\mathbf{i o}}-2 \mathrm{D}$ columnar hexagonal phase with short range intracolumnar order; $\boldsymbol{\Phi}_{\mathbf{s}-\mathbf{0}}^{\mathbf{k}}$ - columnar simple orthorhombic crystalline phase; $\boldsymbol{\Phi}_{\mathbf{c}-\mathbf{0}} \mathbf{k}^{\mathbf{k}}$ - columnar centered orthorhombic crystalline phase; $\boldsymbol{\Phi}_{\mathbf{m}} \mathbf{k}$ - columnar monoclinic crystalline phase. ${ }^{b, c}$ Lattice parameters determined from fiber and powder X-ray diffraction as follows: for hexagonal phases, $d_{\mathrm{hkl}}=\left[4\left(h^{2}+k^{2}+h k\right) /\left(3 a^{2}\right)+(l / c)^{2}\right]^{-1 / 2}$; for orthorhombic phases, $d_{\mathrm{hkl}}=\left[(h / a)^{2}+(k / b)^{2}+(l / c)^{2}\right]^{-1 / 2}$; for monoclinic phases: $d_{\mathrm{hkl}}=\left[(h / a \sin \gamma)^{2}+(k / b \sin \gamma)^{2}+(l / c)^{2}-\left\{(2 h k \cos \gamma) /\left(a b \sin ^{2} \gamma\right)\right\}\right]^{-1 / 2}$. ${ }^{d}$ Column diameter calculated using: $\mathrm{D}_{\text {col }}=a$ for $\boldsymbol{\Phi}_{\mathbf{h}}{ }^{\mathbf{i o}}$ and $\boldsymbol{\Phi}_{\mathbf{h}}{ }^{\mathbf{k}}$, and $\mathrm{D}_{\mathrm{col}}=a /\left[2 \cos \left(\tan ^{-1} b / a\right)\right]$ for $\boldsymbol{\Phi}_{\mathbf{c}-\mathbf{0}} \mathbf{k} .{ }^{e}$ Stratum thickness calculated from the meridional pattern. ${ }^{f}$ Experimental density measured at $23{ }^{\circ} \mathrm{C} .{ }^{g}$ Molecular weight of the compound. ${ }^{h}$ Average number of dendrimers forming the supramolecular column stratum, calculated using: $\mu=\left(\mathrm{N}_{\mathrm{A}} \cdot \rho \cdot \mathrm{A} \cdot t\right) \cdot\left(2 \mathrm{M}_{\mathrm{wt}}\right)^{-1}$, where $\mathrm{N}_{\mathrm{A}}=6.022 \times 10^{23} \mathrm{~mol}^{-1}$, A is the unit cell area of the $a b$-plane, and $t$ is the average stratum thickness calculated from the meridional pattern. ${ }^{i}$ Data taken from ref. ${ }^{1}$. 


\section{Solid State ${ }^{13}$ C CP-MAS NMR of Linear-Racemic Hybrid PBIs}

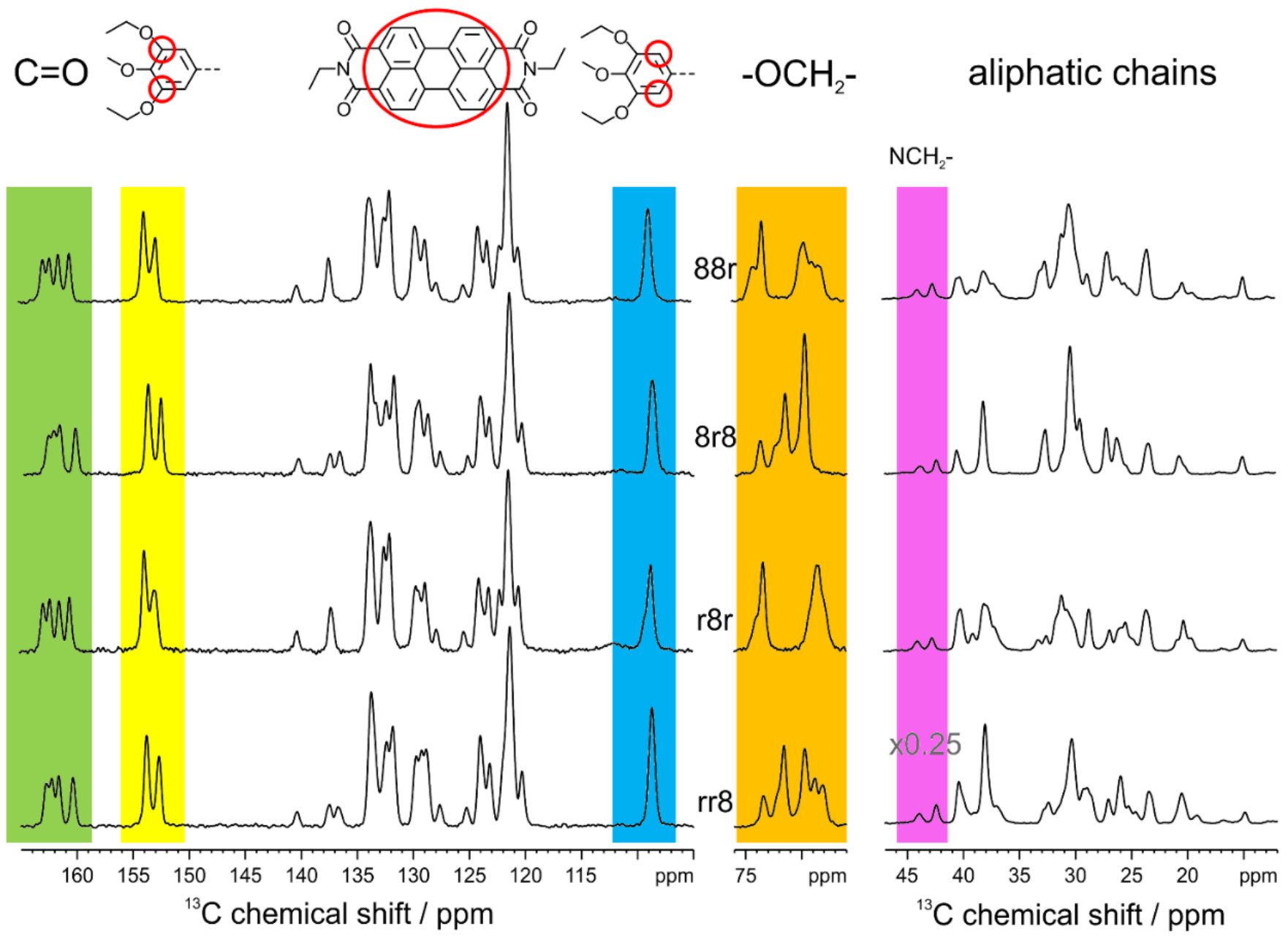

Figure S23. ${ }^{13} \mathrm{C}$ Cross-polarization magic angle spinning (CP-MAS) solid state NMR studies of PBIs with hybrid linear-racemic dendrons. Regions of interest discussed in the main text are colored for ease of reference and correspond to the chemical groups denoted at the top of the figure.

For linear alkyl chains, $\sim 70 \%$ of the $\mathrm{CH}_{2}$ units are significantly more mobile than the $\mathrm{CH}_{2}$ units in branched alkyl chains. The high conformational flexibility of the linear alkyl chains facilitates the intercolumnar space filling and thus the intracolumnar ordering. In particular linear chains in the $m$ - and $p$-positions alter the local mobility of branched side chains, especially at the $\mathrm{CH}_{2}$ groups adjacent to the chiral center. For example, the local order parameter of the $\mathrm{CH}_{2}$ groups adjacent to the stereogenic center decreases from 0.055 in rrr-PBI to 0.047 in r8r-PBI. This decrease in local order parameter indicates higher local mobility at the stereogenic center. This increased local molecular mobility may be crucial to facilitate the ordering of the side chains. Additionally, linear chains may be easier to pack into ordered columns than branched chains, as the latter requires intricate conformational adjustment to direct the chiral center towards the center of the column. These factors together enhance the kinetics of crystallization and support the increased crystallization rate observed for hybrid PBIs vs rrr-PBI. 
7. Representative Models of Linear-Racemic Hybrid PBIs in the $\Phi_{h}{ }^{k 2}$

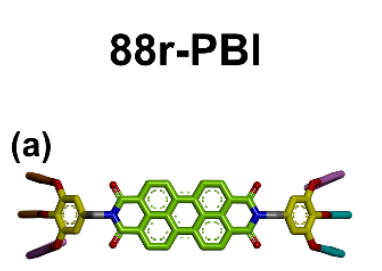

(b)

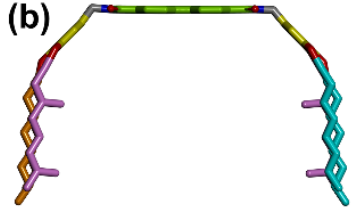

(c)
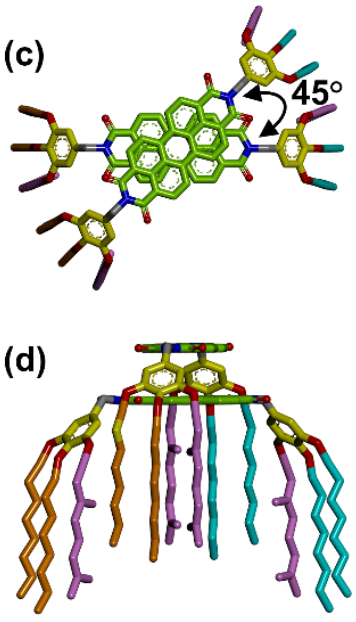

(e)

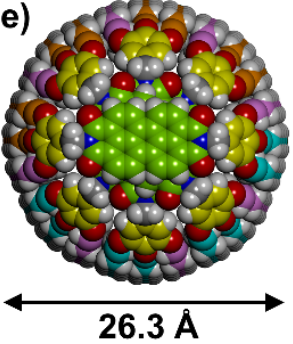

(f)

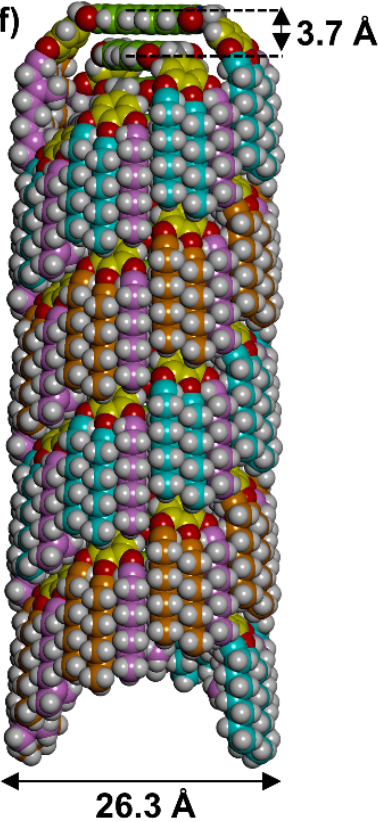

rr8-PBI

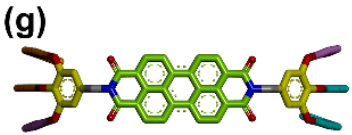

(h)

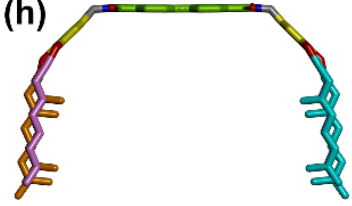

(i)

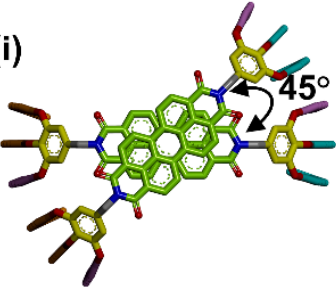

(j)

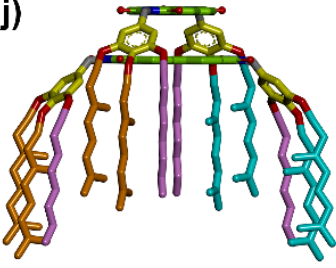

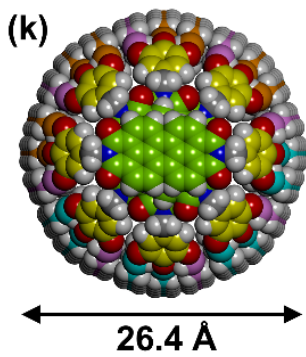

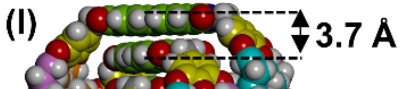

Figure S24. Structural models of (a-f) 88r-PBI and (g-1) rr8-PBI in the $\Phi_{\mathbf{h}}{ }^{\mathbf{k} 2}$ : (a, g) Single molecule, top view; (b, h) Single molecule, side view; (c, i) Dimer, top view; (d, j) Dimer, side view; (e, k) Column, top view; (f, l) Column, side view. Color code: green, carbons of PBI core; yellow, carbons of dendron phenyl; red, oxygen; dark blue, nitrogen; light blue and orange, carbons of majority alkyl chain on opposite dendrons; pink, carbons of minority alkyl chain; H atoms omitted for clarity.

\section{Comment on UV Spectroscopy of Linear-Racemic Hybrid PBIs}

UV data were previously reported for rrr-PBI (Figure S4 in the SI of ref. 10) and compared with literature studies to characterize PBI stacking in its assemblies. In the current work, the combination of identical XRD parameters of the $\boldsymbol{\Phi}_{\mathbf{h}}{ }^{\mathbf{k} 2}$ phase across all hybrid PBIs $(a=26.7 \pm 0.4 \AA$ and $c=14.8 \pm 0.1 \AA)$ and solid state NMR studies (main text and Figure S23) strongly suggest that the differences between the structures of hybrid PBIs lies in the conformation of alkyl chains rather than in the relative stacking of the PBI cores. Hence we expect that the UV data for hybrid PBIs will be indistinguishable from those reported for rrr-PBI. 
9. References for the Supporting Information

(1) Percec, V.; Sun, H.-J.; Leowanawat, P.; Peterca, M.; Graf, R.; Spiess, H. W.; Zeng, X.; Ungar, G.; Heiney, P. A. Transformation from Kinetically into Thermodynamically Controlled SelfOrganization of Complex Helical Columns with 3D Periodicity Assembled from Dendronized Perylene Bisimides. J. Am. Chem. Soc. 2013, 135, 4129-4148.

(2) Heiney, P. A. Datasqueeze: A Software Tool for Powder and Small-Angle X-Ray Diffraction Analysis. Comm. Powder Diffr. Newsl. 2005, 32, 9-11.

(3) Pearson, A. J.; Bruhn, P. R. Studies on the Synthesis of Aryl Ethers Using Arene-Manganese Chemistry. J. Org. Chem. 1991, 56, 7092-7097.

(4) Buzzacchera, I.; Xiao, Q.; Han, H.; Rahimi, K.; Li, S.; Kostina, N. Y.; Toebes, B. J.; Wilner, S. E.; Möller, M.; Rodriguez-Emmenegger, C.; Baumgart, T.; Wilson, D. A.; Wilson, C. J.; Klein, M. L.; Percec, V. Screening Libraries of Amphiphilic Janus Dendrimers Based on Natural Phenolic Acids to Discover Monodisperse Unilamellar Dendrimersomes. Biomacromolecules 2019, 20, 712-727.

(5) Zhang, S.; Xiao, Q.; Sherman, S. E.; Muncan, A.; Ramos Vicente, A. D. M.; Wang, Z.; Hammer, D. A.; Williams, D.; Chen, Y.; Pochan, D. J.; Vértesy, S.; André, S.; Klein, M. L.; Gabius, H.-J.; Percec, V. Glycodendrimersomes from Sequence-Defined Janus Glycodendrimers Reveal High Activity and Sensor Capacity for the Agglutination by Natural Variants of Human Lectins. J. Am. Chem. Soc. 2015, 137, 13334-13344.

(6) Percec, V.; Imam, M. R.; Peterca, M.; Leowanawat, P. Self-Organizable Vesicular Columns Assembled from Polymers Dendronized with Semifluorinated Janus Dendrimers Act As Reverse Thermal Actuators. J. Am. Chem. Soc. 2012, 134, 4408-4420.

(7) Percec, V.; Imam, M. R.; Peterca, M.; Wilson, D. A.; Heiney, P. A. Self-Assembly of Dendritic Crowns into Chiral Supramolecular Spheres. J. Am. Chem. Soc. 2009, 131, 1294-1304.

(8) Percec, V.; Peterca, M.; Tsuda, Y.; Rosen, B. M.; Uchida, S.; Imam, M. R.; Ungar, G.; Heiney, P. A. Elucidating the Structure of the $P m 3 n$ Cubic Phase of Supramolecular Dendrimers through the Modification of Their Aliphatic to Aromatic Volume Ratio. Chem. Eur. J. 2009, 15, 8994-9004.

(9) Percec, V.; Peterca, M.; Tadjiev, T.; Zeng, X.; Ungar, G.; Leowanawat, P.; Aqad, E.; Imam, M. R.; Rosen, B. M.; Akbey, U.; Graf, R.; Sekharan, S.; Sebastiani, D.; Spiess, H. W.; Heineu, P. A.; Hudson, S. D. Self-Assembly of Dendronized Perylene Bisimides into Complex Helical Columns. J. Am. Chem. Soc. 2011, 133, 12197-12219.

(10) Roche, C.; Sun, H.-J.; Leowanawat, P.; Araoka, F.; Partridge, B. E.; Peterca, M.; Wilson, D. A.; Prendergast, M. E.; Heiney, P. A.; Graf, R.; Spiess, H. W.; Zeng, X.; Ungar, G.; Percec, V. A Supramolecular Helix That Disregards Chirality. Nat. Chem. 2016, 8, 80-89. 